Historic, Archive Document

Do not assume content reflects current scientific knowledge, policies, or practices. 


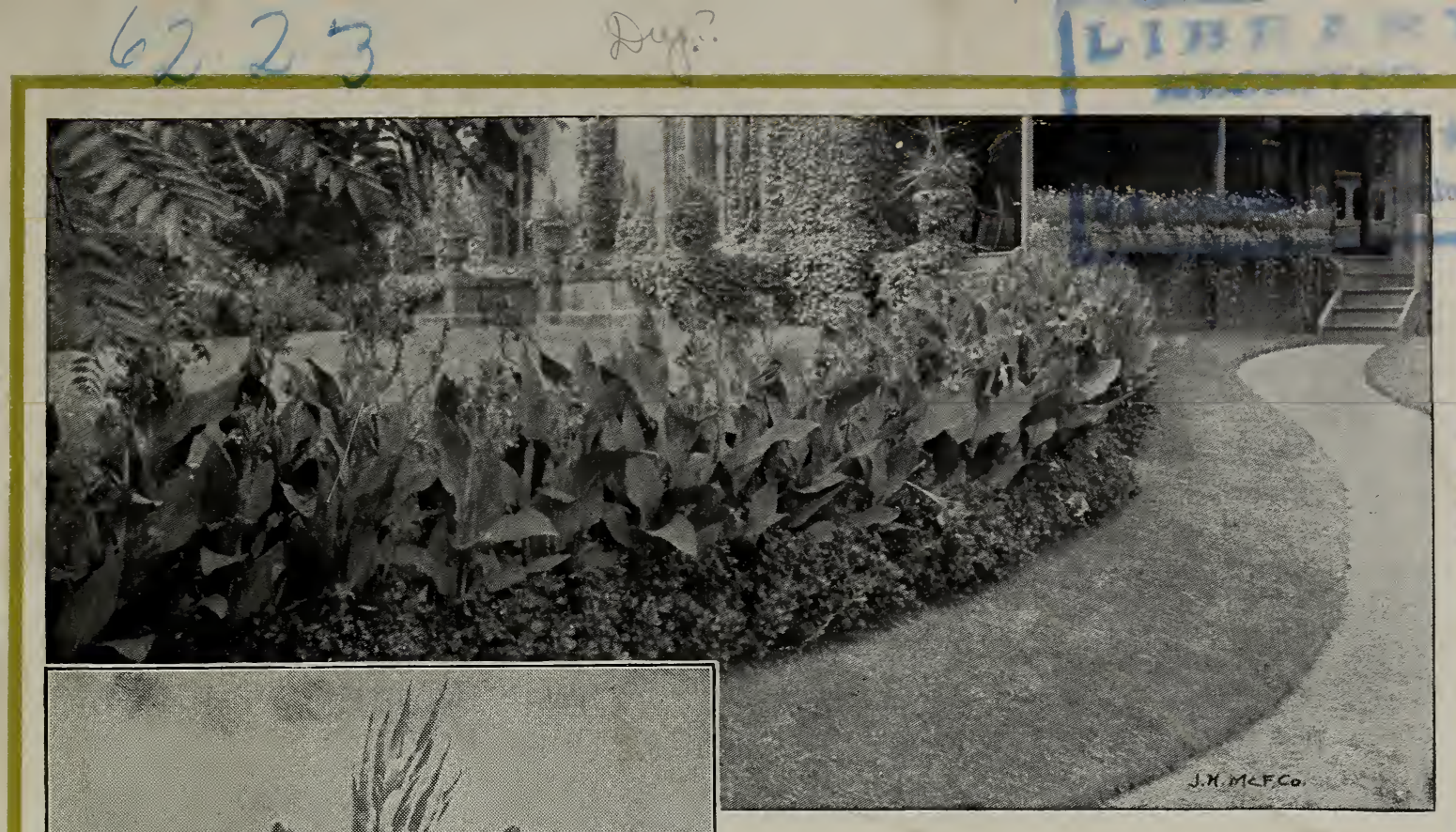

\section{Wholesale Price-list}
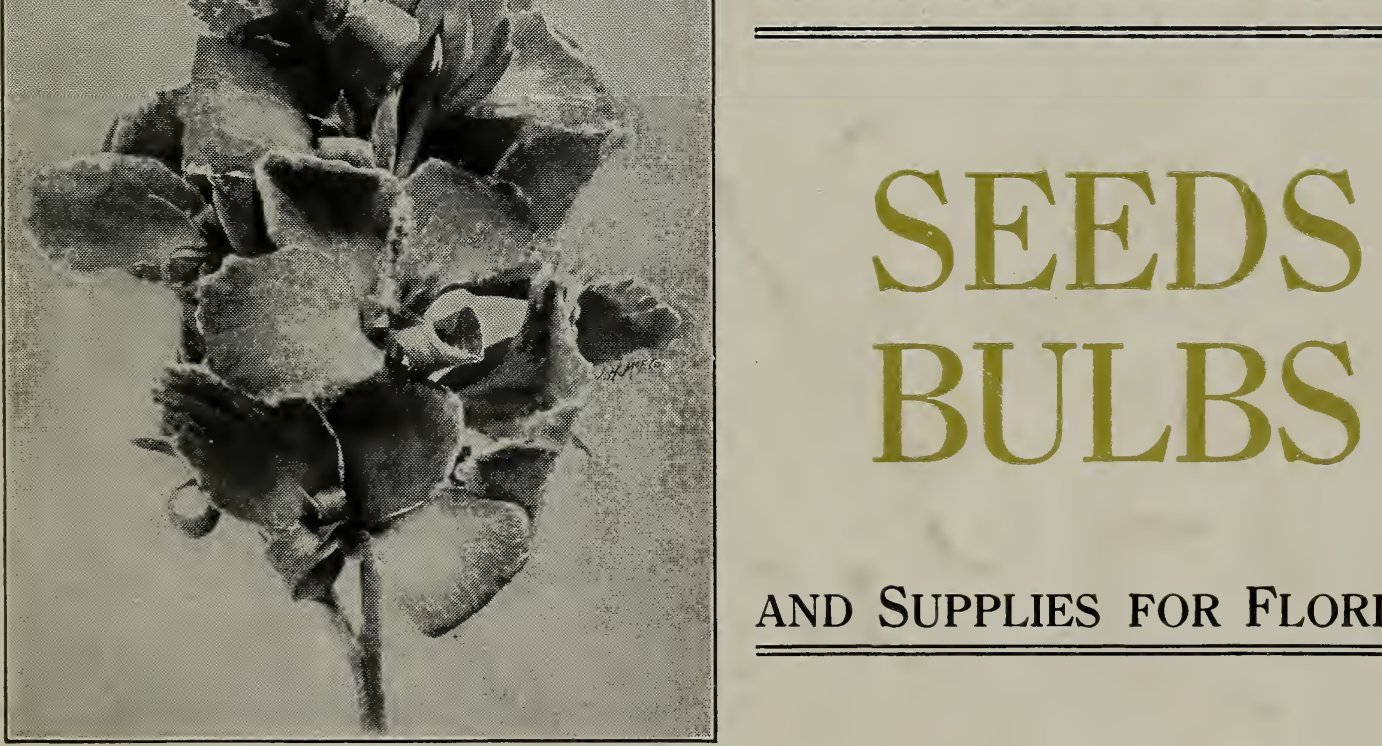

AND SUPPLIES FOR FLORISTS

$\underline{\text { SPRING } \cdot 1916}$

\section{THE W. W. BARNARD COMPANY}

231-235 W. Madison Street (near Fifth Avenue) 


\section{FLORISTS WHOLESALE LIST of Seeds, Bulbs, Dormant Plants and Supplies}

\section{TO THE TRADE}

CONSTANTLY increasing business brings with it a demand for additions to almost every line of stock. Under the separate heads of Flower Seeds, Bulbs, Dormant Plants and Sundries will be found a number of new introductions, as well as the time. tried stand-bys. The prices have been revised and supersede all others in previous lists.

It is our endeavor to get stock to you at the right time. YOU can assist us by making your purchases early. Delayed orders sometimes arrive when our supply is depleted, and occasionally sold out.

As heretofore, QUALITY with us is the first consideration. With full stocks and every facility to execute your orders carefully and well, we ask the pleasure of supplying your needs.
TERMS OF SALE

A LL general bills of Flower Seeds, Bulbs, Plants A and Supplies (except as otherwise stated) are payable 60 days from date of invoice, subject to 2 per cent discount from prices in this book for cash in 10 days.

Fertilizers, Grass Seeds, Green, Holly, Tin Foil, net cash and subject to fluctuations of market.

Special terms, when desired, will be made by correspondence on large orders. Unknown correspondents desiring credit are requested to furnish satisfactory references. C. O. D. orders should be accompanied by one-third of amount of purchase.

Shipping Directions should accompany each order. State definitely if you wish us to send by express or freight. In absence of instructions we reserve right as to route and manner of transportation.

No charge for packing, cartage nor delivery to any transportation company in Chicago.

WHILE we exercise great care to have all Seeds, Bulbs and Plants pure and reliable, we give no warSeeds, Bulbs and Plants we send out, and we will not be in any way responsible for the crop. If the purchaser does not accept the goods on these terms they are at once to be returned.

\section{GRASS SEED FOR LAWNS, PLEASURE GROUNDS, ETC.}

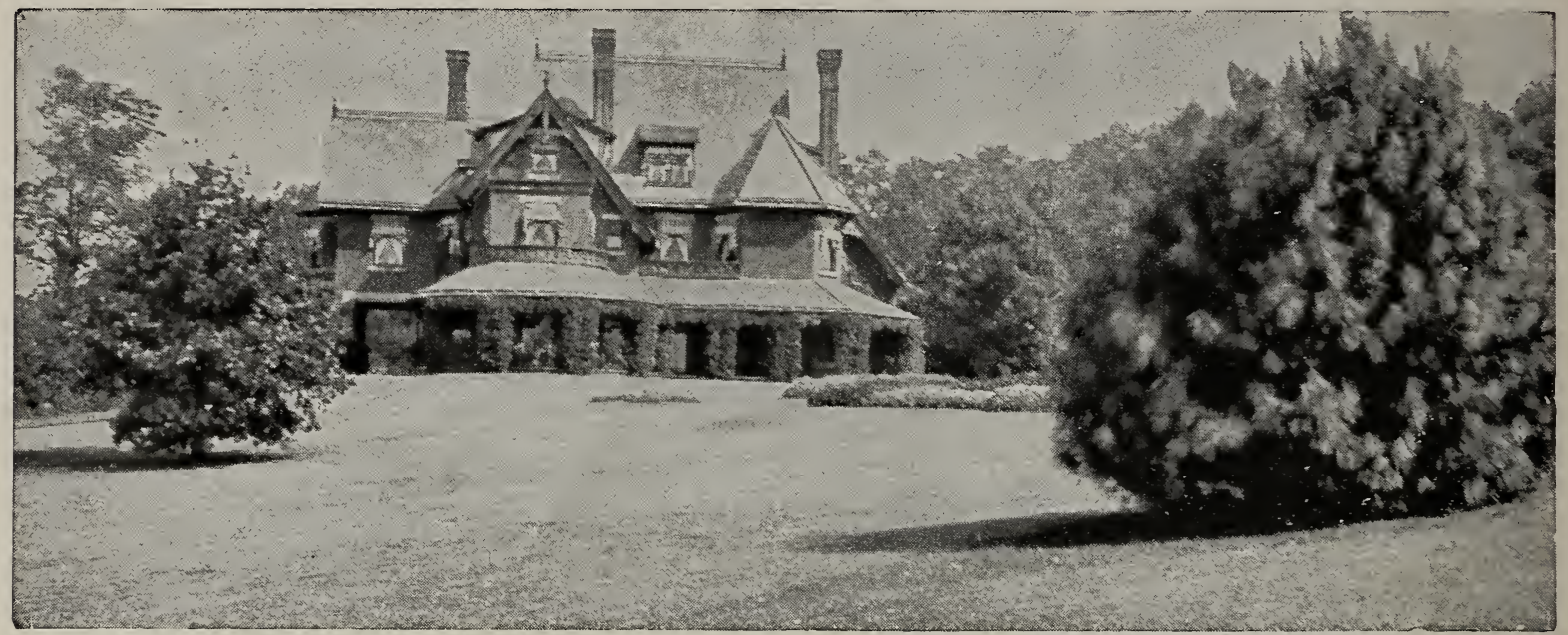

\section{"Perpetual Green" Lawn Mixture}

A combination of various native and foreign dwarf-growing. fine-leaved grasses, carefully balanced and blended, with a view of producing a turf that will retain its rich green color throughout the entire season. This is our standard mixture that we have sold for years to parks, cemeteries and large private estates. Put up in $1 / 2-1 b$. and $1-1 b$. cartons; also in 5,7 and $14-1 \mathrm{~b}$. cloth bags. Price, in cartons, per lb., 18c; in bulk, $17 \mathrm{c}$. Retail price, $1 \mathrm{~b} ., 30 \mathrm{c}$.

\section{Choice Mixture}

While containing fewer and less expensive grasses than our "Perpetual Green," this is a good, wellbalanced mixture which we do not hesitate to recommend. Same containers as above. Price, in carons, per lb., 13c; in bulk, 12c. Retail price, 1b., 25c.

\section{Barnards' "Special" Lawn Mixture}

This mixture is composec of grasses selected irrespective of cost as representing the best low-growing, narrow-leaved varieties of fine texture and color. In preparing this "Special" mixture only seeds of the highest grades and extra heavy weight are emploved. It costs more, but "it's worth more." Seeded liberally upon good ground, well prepared a lawn of the very highest excellence will result. In bulk only, per lb., 25c. Retail price, ib., $40 \mathrm{c}$.

\section{Terrace Mixture of Grasses}

For seeding side hills, terraces, embankments, ete. A mixture of grasses whose roots penetrate deeply and bind the soil. When sowing, add to the mixture about one-fifth part of oats, which holds the soil together until the grasses have made a good start. In bulk only, per lb., 20c. Retail, 1b., 30c.

\section{Shady Place Mixture}

Made up of grasses selected for locations shaded by trees or buildings. In lb. carton, $25 \mathrm{c}$; bulk, $23 \mathrm{c}$. Retail price, lb., $35 \mathrm{c}$

\section{Putting Green Mixture}

This mixture is intended not only for putting greens, but for all lawns that are to be used as pleasure grounds and necessarily requiring a fine, dense turf-one capable of withstanding constant wear. With this in view, after experimenting with several combinations of the best fine-leaved $10 \mathrm{w}$ growing grasses. we selected those which, rightfully proportioned together, produced just the results we were endeavoring to obtain. We have furnished some of the best known golf clubs in the vieinity of Chicago with this mixture and it has given splendid results. Price, per pound, $35 \mathrm{c} ; 5 \mathrm{lbs}$. for $\$ 1.50$. Retail price, lb., $45 \mathrm{c}$.

\section{Mixture for Quick Effect}

There is considerable demand for a grass seed that will produce a quick-growing turf. For this purpose we have prepared a mixture of a few strong-germinating, vigorous-growing grasses, which will produce a turf of fair quality within a few weeks after seeding. The grasses in this mixture are mostly annuals or biennials, and such a turf could be relied upon onlv for one or two seasons. A permanent lawn could be secured, however. by re-seeding at any time with any of our other mixtures. In carton, 11 cts.; in bulk, lb., 10 cts. Retail, lb., 20 cts.

\section{White Clover}

Very scarce this season. Extra fancy quality. Lb., 50 cts.; $5 \mathrm{lbs}$. for $\$ 2.25$. Retail price, per lb., 75 cts. 


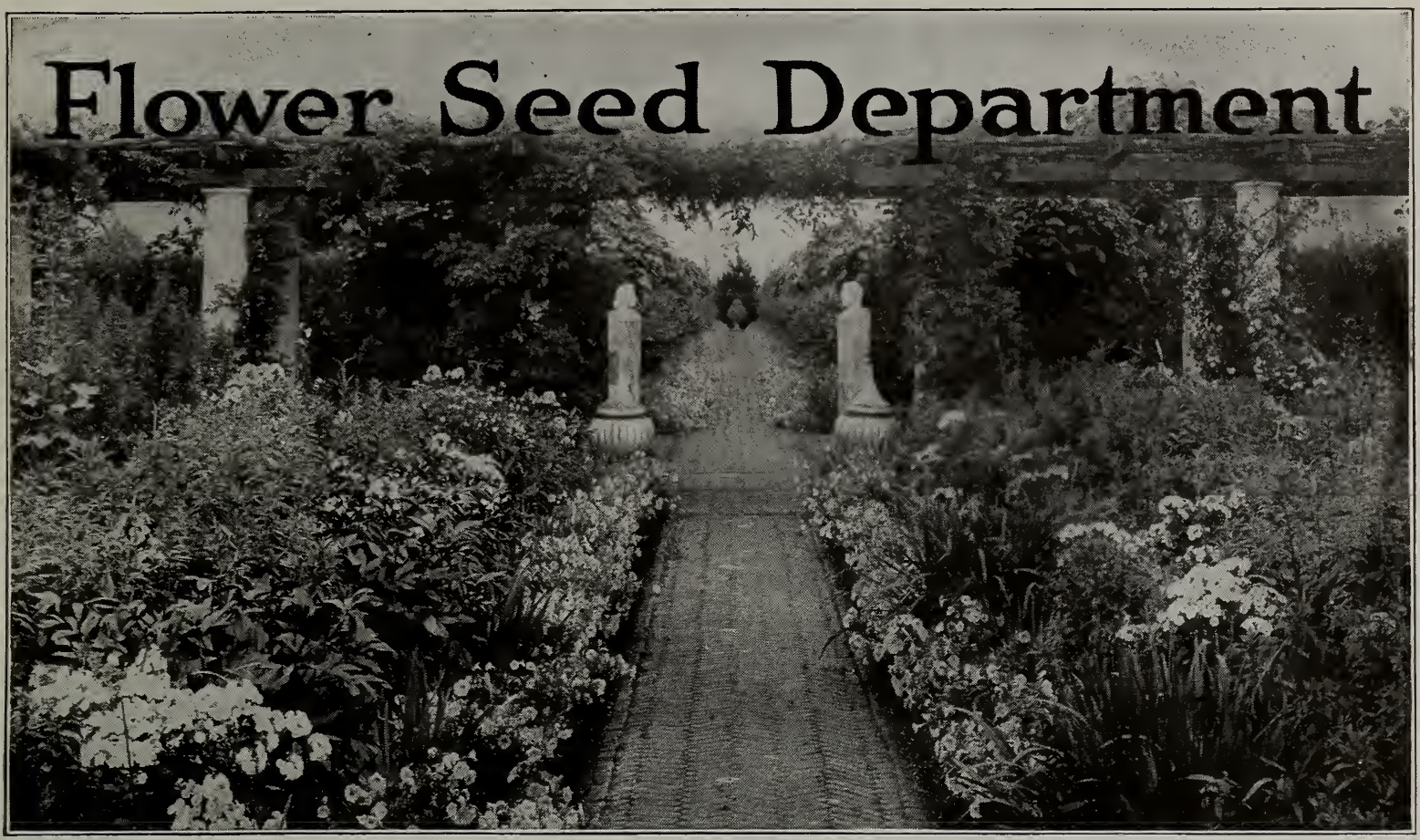

It is generally conceded that choice flowers, marketed early, not only sell easily but bring the top price on the market. These conditions, so much to be desired by every producer of flowers, have their foundation in seeds something better than the ordinary strains in commerce. Our long business relations with reliable seed growers enables us to secure their best stocks. We offer quality seeds, second to none and equal to any.

PREPAID. We prepay postage on all flower seeds in trade packets and ounces. Postage extra on one-fourth pound or over. Rate, 8 cents per pound.

TERMS and CASH DISCOUNT.-All general bills of Seeds are payable 60 days from date of invoice. On Flower Seeds a special discount of 10 per cent is granted when cash accompanies the order.

TRADE PACKETS contain liberal quantities in proportion with the price per ounce, and are sent on all orders unless otherwise specified. Where smaller quantities than Trade Packets are desired, Retail Packets may be ordered at a discount of $33^{\mathrm{T}} / 3$ per cent from retail prices.

Biennials * Perennials $\dagger$ Trade pkt. Oz.

ABRONIA umbellata, trailing plant, pink..........\$0.05 $\$ 0.35$

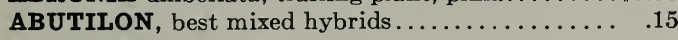

†ACHILLEA, "The Pearl," double white........ .20

ACONITUM Napellus, blue............... .15

ACROCLINIUM (Everlasting Flower). Mixed..... .05

*ADLUMIa cirrhosa (Allegheny Vine)......... .15

AFRICAN DAISY. See Dimorphotheca.

AGATHEA cœlestis (Blue Daisy)............ . .10

AGERATUM. For pots and bedding.

Dwarf Blue............. 10

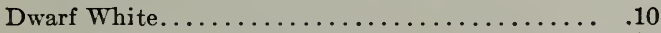

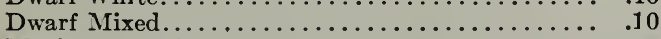

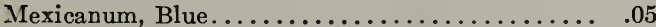

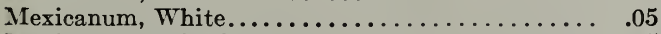

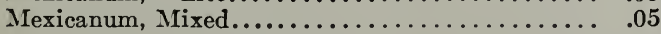

Blue Perfection. Fine dark blue............. .10

Little Blue Star. Light blue............... .35

ALONSOA grandiflora.................... .05

AI YSSUM. Fine summer bedding plant.

Maritimum (Sweet Alyssum). White...lb. \$1.20. . .05

Carpet of Snow. Useful for edgings............ .10

Little Dorrit. Compact, bushy............... . .10

Little Gem, Miniature. Very dwarf ............ .10

4Saxatile. Yellow; spring-blooming............. $\quad .10$

AMARANTUS caudatus. Dark red........... .05

Tricolor (Joseph's Coat).....................

Many varieties in mixture........................ .05

†AMPELOPSIS Veitchii (Japan Ivy)....lb. \$1.75. . .10

†ANCHUSA Italica. Blue flowers.............. .15

†ANTHEMIS Kelwayi. Yellow................ .10

†ARIS TOLOCHIA Sipho................. .10

ASCLEPIAS tuberosa. Orange.............. .15

4ANEMONE coronaria. Mixed................

St. Brigid's Hybrids ........................
Biennials * Perennials $\dagger$

†ANTIRRHINUM (Snapdragon).

Dwarf, all colors, mixed................. \$0.05 $\$ 0.30$

Dwarf, Daphne. Carmine....................

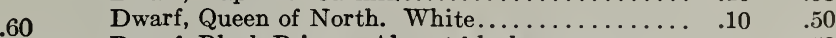

$\begin{array}{llll}.15 & \text { Dwarf, Black Prince. Almost black............... } & .10 & .50 \\ \text { Dwarf, separate colors, White, Carmine, Pink, each } & .10 & .45\end{array}$

ANTIRRHINUM, Tall Varieties.

Silver Pink. Very desirable.............. .40

Venus. Pink shade on white ground .........

Purple King. Deep purple.............. .15

The Bride. White and rose.............. 15

Tall, separate colors, White, Carmine, Coral-Red,

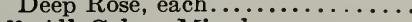

Tall, All Colors Mixed..........................

Giant-flowered, Mixed......................

Queen Victoria. White flowers...............

†AQUILEGIA (Columbine).

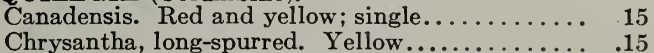

Chrysantha, Double. Yellow..................

Cœrulea hybrida. White and blue...................

Cœrulea.......................................

Grandiflora alba. Half-dwarf; white........... $\quad .20$

Nivea grandiflora. Large; pure white........... .10

Formosa pl. Double blue...................

Formosa. Double; white.................. .10

Double Red and Double Rose. Each............ 10

Double, mixed colors.............................$_{05} \quad .30$

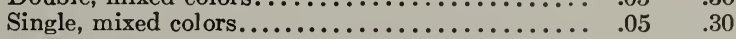

ARTEMISIA (Summer Fir). Forms bushes 4 to 5 feet high. Suitable for hedges or groups; similar to Kochia. Also desirable for pot-culture. Trade pkt., $15 \mathrm{cts}$. T. pkt. Oz. ARCTOTIS grandis (Lilac Daisy)............ $\$ 0.05 \quad \$ 0.25$

ASPARAGUS plumosus nanus. Greenhouse-grown; extra quality. 100 seeds, 50 cts.; 250 seeds, $\$ 1 ; 1,000$ seeds, $\$ 3.50$.

Sprengeri. Best quality. 100 seeds, 15 cts.; 1,000 seeds, 75 cts. 


\section{ASTERS of Superior Quality}

For several years we have been making trials at jur nursery of the many classes and strains of Asters now in cultivation. We believe the list which follows contains the choicest sorts. The seed we send out was grown by experienced specialists both at home and abroad-some of it under our personal supervision. Seed of highest quality is cheapest in the end. We handle none other than the best.

Note.-Asters are priced by Trade Packet, 1/4 oz. and oz. Carlson's and some others by $1 / 8 \mathrm{oz}$. and oz.

\section{Carlson's Improved or Invincible}

Plants of branching habit, bearing paeony-formed flowers of large size and fine substance. Healthy; prolific; in bloom until checked by frost.

The seed we sell was grown by originator.

White. Light pink. Lavender.

Rose pink. Crimson. T. Pkt. $1 / 8 \mathrm{Oz}$ Separate colors, each............\$0.20 \$0.30

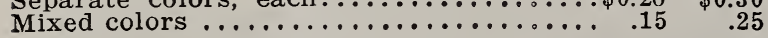

\section{ASTER, PINK ENCHANTRESS}

The flower is always full double; petals are of medium length and very broad. Those toward the center fold in, while those toward the outer edge stand out more and more, thus making the bloom develop its exceedingly large size. A grand Aster. Trade pkt. $20 \mathrm{c}$; $1 / 4$ oz. $50 \mathrm{c}$.

\section{GIANT COMET, BRANCHING}

These have large flowers and long petals.

Snow-White. Crimson. Lavender.

Carmine. $\quad$ Dark Blue. Rose.

T. Pkt. 1/4 Oz. Oz.

Separate colors, each..............\$0.20 \$0.50 $\$ 1.50$

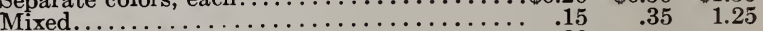

Empress Frederick, white............. .20

\section{ASTER, CREGO}

Without a doubt, the Crego Asters are the most popular on many markets.

Careful selection and skill have resulted in plants of fine, sturdy growth, well branched. Fluffy, graceful flowers, 4 to 5 inches across. The cut blooms last well. Dark pink, shell-pink, purple, white, mixed. Trade pkt. (about 500 seeds) 20c.; $1 / 4 \mathrm{oz} .50 \mathrm{c}$.; oz. $\$ 1.75$.

We use only originator's strain of seed.

\section{The DAYBREAK Group}

Every florist who has grown the beautiful Asters, Daybreak and Purity, will hail with pleasure the opportunity now afforded to secure some entirely new colors in this fine class.

DAYBREAK. One of the most beautiful Asters in existence. In color a delicate shell pink. Trade pkt. $20 \mathrm{c}$.

PURITY. This snow white Aster is similar to Daybreak, except in color. Trade pkt. $20 \mathrm{c}$.; $1 / 4$ oz. 50 c.

SALMON KING. Color is novel and pleasing shade, bright enough to be conspicuous. Trade pkt. $20 \mathrm{c}$

ROSE KING. Trade pkt. 20 c.; $1 / 4$ oz. 50c.

LEMON DROP. A yellow one at last! It is a decided yellow. Flowers not quite so large as its parent, Daybreak. Trade pkt $20 \mathrm{c}$; $1 / 8$ oz. $45 \mathrm{c}$.

ROSY MORN. Large, well-formed flowers of a handsome shade of rose. One of the best of the group. Trade pkt. 20c.; $1 / 8 \mathrm{oz}^{\circ}$ $40 \mathrm{c}$

LAVENDER GEM. Loose flowers of that pleasing shade of lavender so much admired. Trade pkt. $20 \mathrm{c} . ; 1 / 8$ oz. $50 \mathrm{c}$.

VIOLET KING. In color is a soft violet lilac. In form the flower is entirely distinct, petals somewhat quilled but large and broad. Round. Trade pkt. 20c.; $1 / 8$ oz. $30 \mathrm{c}$.

\section{Other Varieties of Asters}

TRIUMPH OF PARIS. New. Produces flowers four to six inches across, with long, pure white outer petals, in the center the quilled florets form a bright golden wreath. Trade pkt. $20 \mathrm{c}$.

CACTUS. Carmine flowers with twisted petals. Cut flowers are very lasting. Trade pkt. 25c.

CARDINAL. In growth resembling the Branching Aster, but a little more dwarf; in color a beautiful shade of cardinal. Commences to bloom in August. Trade pkt. 20c.; $1 / 4$ oz. $75 \mathrm{c}$.

CRIMSON GIANT. A splendid upright-growing sort, producing very large, well-formed, dark-crimson flowers. Its brilliancy of color and erect habit make it desirable for garden culture. Trade pkt. 20 c.; $1 / 8$ oz. 40 c.
CREGO ASTER AS GROWN BY ORIGINATOR

\section{Other Varieties of Asters}

MIKADO PINK. Of branching habit; flowers of an exquisite shade of shell-pink; the petals are long, narrow and twisted. Trade pkt. $15 \mathrm{c} ; 1 / 4$ oz. $40 \mathrm{c}$.

MITADO WHITE. Trade pkt. $15 \mathrm{c} ; 1 / 4$ oz. $40 \mathrm{c}$

ROYAL PURPLE. Large flowers with full centers and of a rich shade of royal purple. Plants of branching habit and medium to late flowering. Trade pkt. $15 \mathrm{c} ; 1 / 4$ oz. $50 \mathrm{c} ;$ oz., $\$ 1.50$.

ROYAL STRIPE. Of Giant Comet type. Large flowers on long stems. A broad band of violet blue runs the entire length of each petal. A beautiful Aster. Trade pkt. 20c; 1/8 oz., $60 \mathrm{c}$. 


\section{ASTERS-Continued}

Note.-Asters are priced by Trade Packet, $3 / 4 \mathrm{oz}$. and oz. Carison's and some others by $1 / 8 \mathrm{oz}$. and oz.

\section{DWARF BOUQUET-Height 8 Inches}

T. Pkt. ea., 10c. 1/4 oz. oz. Pink …. \$0.35 $\$ 1.25$ White ...... $\$ 0.35 \$ 1.25$ Dark biue ....... 155 1.25 Crimson $\ldots . . . \quad .35 \quad 1.25$ Bouquet, mix; ${ }^{2} .35 \quad 1.25$

DWARF Chrysanthemum Aster,

mixed ................ $\$ 0.15$

DWARF QUEEN (Height 10 inches).

For pots and beds. Early, White, crimson, mixed, each........ .15

\section{HOHENZOLLERN}

An excellent, robust, growing class with enormous flowers borne upon long stems. A splendid cut flower.

T. Pkt. ea., 15c. 1/4 oz. oz.

White $\ldots . . . \$ 0.70 \$ 2.50$

Light blue.... $\quad .70 \quad 2.50$

T. Pkt. ea., 15c. 1/4 oz. oz. Rose ....... $\$ 0.70 \$ 2.50$ Lavender … $\quad .70 \quad 2.50$ Mixed ......... $\quad .50 \quad 1.75$ Dark blue.... $.70 \quad 2.50 \quad$ Mixed ........ .50 1.75 of enormous size. (Novelty 1915.) Trade pkt. 15c.

HERCULES. Huge, elegant flowers; mixed colors. Seed in demand and scarce. Trade pkt. 35c.

MAUVE QUEEN. A mammoth Aster of Ostrich Feather type. Pearl-gray in color. Td. pkt. 25c. OSTRICH FEATHER. White, pink, each, Trade pkt. $20 \mathrm{c} ; 1 / 4$ oz, $50 \mathrm{c}$.

Mixed, trade pkt. $15 \mathrm{c} ; 1 / 4$ oz. $45 \mathrm{c}$.

\section{QUEEN OF THE MARKET}

Blooms three weeks earlier than other Asters. $\begin{array}{lll}\text { Blooms three weeks earlier than other } & \\ \text { White } & \text { Light blue Purple }\end{array}$
Crimson
Pink
Lavender

T. Pkt. $1 / 4$ oz.

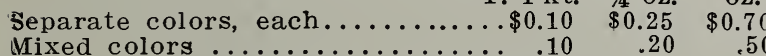

oz.

\section{SEMPLE'S BRANCHING}

The form of the flowers, which are borne upon long stems, places this class of Asters as among the very best late blooming varieties for cut flowers.

T. Pkt. ea., 10c. T/4 oz. oz. T. Pkt. ea., 10c. 1/4 oz. oz. White..........\$0.25 $\$ 0.70$ Crimson........\$ \$0.25. $\$ 0.70$

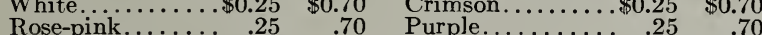

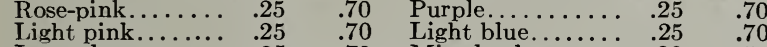
Lavender.......... $\quad .25 \quad .70$ Mixed colors........

TRUFFAUT'S (Paeony-Flowered) PFRFECTION.

Large flowering asters; grow about 18 in. tall. T. Pkt. ea., 20c. 1/4 oz. oz. T. Pkt. ea., 20c. 1/4 oz. oz. White ...... \$0.45 $\$ 1.50$ Light blue.... $\$ 0.45 \$ 1.50$ Rose ......... $45 \quad 1.50$ Dark blue.... $4.45 \quad 1.50$ $\begin{array}{llllll}\text { Rose } \ldots \ldots \ldots & .45 & 1.50 & \text { Dark blue.... } & .45 & 1.50 \\ \text { Blood red.... } & .45 & 1.50 & \text { La Superb. } & & \\ \text { Crimson } \ldots \ldots & .45 & 1.50 & \text { sky blue.... } & .45 & 1.50 \\ & & & \end{array}$

$\begin{array}{rrrrrrr}\text { Crimson } & \ldots . & .45 & 1.50 & \begin{array}{r}\text { sky blue... } \\ \text { Mix. pkt. } 15\end{array} & .45 & 1.50 \\ & & & .35 & 1.25\end{array}$

\section{IMPROVED VICTORIA}

Medium in height. Large flowers of great beauty.

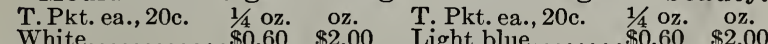
White..........\$ \$0.60 \$2.00 Light blue.......\$0.60 \$2.00

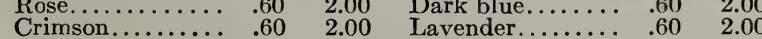
$\begin{array}{lllllll}\text { Crimson.......... } & .60 & 2.00 & \text { Lavender.......... } & .60 & 2.00 \\ \text { Mixed colors...... } & .60 & 2.00\end{array}$

\section{SMITH'S PEERIESS}

Habit of growth similar to the branching sorts. The stems are long and wiry; flowers of large size, heavily petalled. A grand cut flower Aster.

Peerless White, Trade pkt. ..............\$2 . $\$ 0.25$

Peerless Pink, Trade pkt. .....................25

VICK'S BRANCHING. A late blooming class.

T. Pkt. ea. 10 c. $1 / 4$ oz. oz. $\mid$ T. Pkt. ea. 10 c. $1 / 4$ oz. oz. White ...... $\$ 0.35 \$ 1.00$ Crimson …. $\$ 0.35 \$ 1.00$

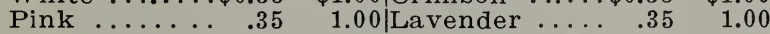
$\begin{array}{lllll}\text { Purple } \ldots . . .6 & .35 & 1.00 \mid \text { Mixed colors... } & .30 & .90\end{array}$ Rose ........... .35 1.00

$$
\text { T. Pkt. } 1 / 4 \mathrm{oz} \text {. }
$$

TALL ASTERS, mixed colors . . . $\$ 0.10 \quad \$ 0.20 \quad \$ 0.40$ DWARF ASTERS, mixed colors. . . .10 $\quad .30 \quad 1.00$ HA RDY ASTERS, mixed colors... .15

\section{ASTERMUM}

The originator says "Each plant yields from ten to fifteen blooms and their solidity is such that they make good shippers." White, pink, lavender. Each
†AURICULA (Primula), Mixed...............\$0.25

BALSAM. Double mixed..................... .10 \$0.69

White Perfection. The best double, pure white Balsam for florists' use.................... .10

Double, separate colors, Scarlet, White, Rose, Yel-

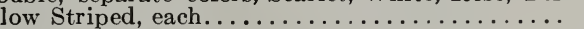

BALLOON VINE

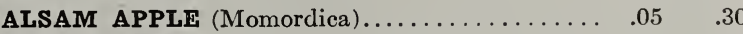

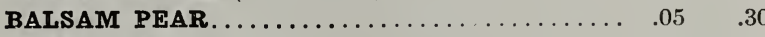

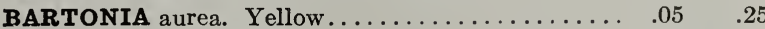

\section{BEGONIAS}

The following varieties, excepting Rex, are now extensively used for bedding, for which purpose they are admirably adapted. Sow in February for young plants to bed out in June. Early sown seed blooms the first season:

Semperflorens alba, trade pkt. 10c.

Erfordia, pink, fine bedding sort, trade pkt., $25 \mathrm{c}$. Rex, ornamental leaved, trade pkt. 50c.

Vernon dark green leaves, $1 / 4$ oz. $\$ 1.00$; tde. pkt. $10 \mathrm{c}$ Vernon, Dwarf, prized for bedding, tde. pkt. $25 \mathrm{c}$.

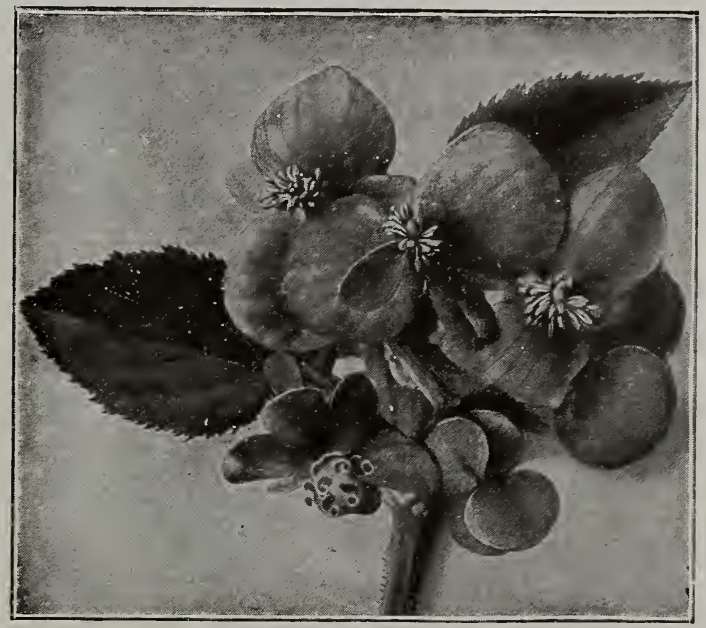

BEGONIA GRACILIS.

This is one of the best introductions of recent years. It is equally useful for bedding or conservatories; vigorous grower and bloomer.

Prima Donna, light rose. Trade pkt. $20 \mathrm{c}$

Luminosa, fiery scarlet. Trade pkt. $25 \mathrm{c}$.

Mignon. A grand bedder; 7 to 8 inches high; dark green foliage, scarlet flowers with green anthers. Makes nice bushy plants. Trade pkt. $25 \mathrm{c}$.

\section{BEGONIAS-Tuberous-Rooted}

We offer distinctly new and improved varieties far superior to those generally grown.

Victoria. Scarlet flowers in various tints. Petals fringed and curled, producing a fine effect. Trade pkt. $50 \mathrm{c}$.

Single, tuberous, mixed. Trade pkt. $50 \mathrm{c}$. Double, tuberous, mixed. Trade pkt. 50c.

PBELLIS PERENNIS (Double Daisy).

Monstrosa. This strain brings flowers almost as large as ordinary Zinnias. They are borne on

long, strong, stiff stems. T. Pkt. Oz.

Montrosa Daisy, white ..........\$0.25

Montrosa Daisy, pink ...................

Longfellow, double rose $\ldots \ldots \ldots \ldots \ldots \ldots$

Snowball, double white $\ldots \ldots \ldots \ldots \ldots \ldots . .15 \times \ldots \ldots$

Giant, white

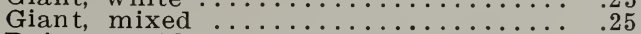

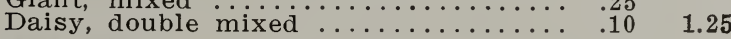

Shasta Daisy ............................. .15 1.50

BOLTONIA asteroides, flesh-colored . . . . . . . . 25

Latisquama, white ........................... $\quad .25$

BRACHYCOME iberidifolia ................ . 10

BROWALLIA, Mixed $\ldots \ldots \ldots \ldots \ldots \ldots \ldots \ldots . .10$

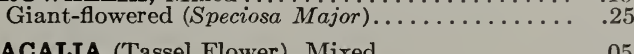

CACALIA (Tassel Flower), Mixed ............ . .05 $\quad .25$ 


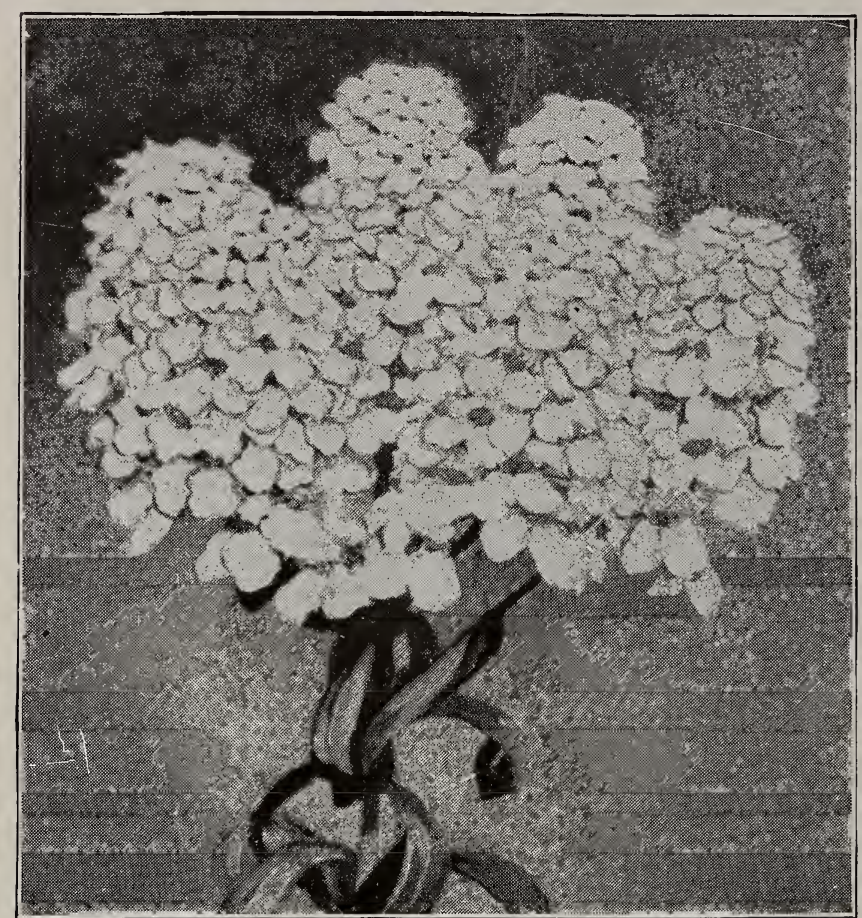

CANDYTUFT. GIANT HYACINTH-FLOWERED

CALCEOLARIA hybrida grandiflora.

Hybrida pumila. Dwarf-growing .

CALENDULA (Pot Marigold).

Meteor. Rich orange.

Prince of Orange. Pale yellow

Mixed.

Orange King.

CALLIOPSIS (Coreopsis).

Drummondii (Golden Wave)....

Mixed

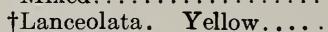

T. Pkt. Oz

$\$ 0.50$

.50

$.05 \quad \$ 0.15$

$.05 \quad .15$

$.05 \quad .15$

$.05 \quad .15$

.05

.05

†CAMPANULA (Bellflower).

Single, Mixed.

Single, Blue, White, Rose.

Double, Mixed...

Double, Blue, White, Rose

Cup and Saucer, Mixed.

Blue, White, Rose

Pyramidalis, Blue, White.

Persicifolia grandiflora, White .

Persicifolia grandiflora, Blue.

CANARY-BIRD VINE

CANDYTUFT, Hyacinth-flowered. White.

White Rocket. A good sort.

Empress. Handsome; large; pure white.

Little Prince. Very dwarf; white... .

Queen of Italy. Pink.

Tom Thumb. Dwarf; white. . .

Carmine.

Crimson, Lilac.

All kinds, mixed.

†Sempervirens. White

tGibraltarica. Lilac, shaded white

tCARNATION, Chaubaud's Perpetual

Double, Hardy. Finest mixed......

Grenadin, Red. Blooms in summer....

Grenadin, White. Excellent for florists

Giant Margaret. Mixed. .

Margaret, Mixed Colors.

Margaret, White. ...

Margaret, Pink, Red.

CELOSIA, Pres. Thiers. Red combs

Tall Cockscomb. Choice Mixture.

Dwarf Cockscomb.

Ostrich Feather, Crimson....

Ostrich Feather, Yellow ...

Ostrich Feather, Mixed.

Triumph of the Exposition. Crimson

Thompsonii magnifica. Fine feathered

CANNA, Crozy. Mixed....

Tall Mixed...

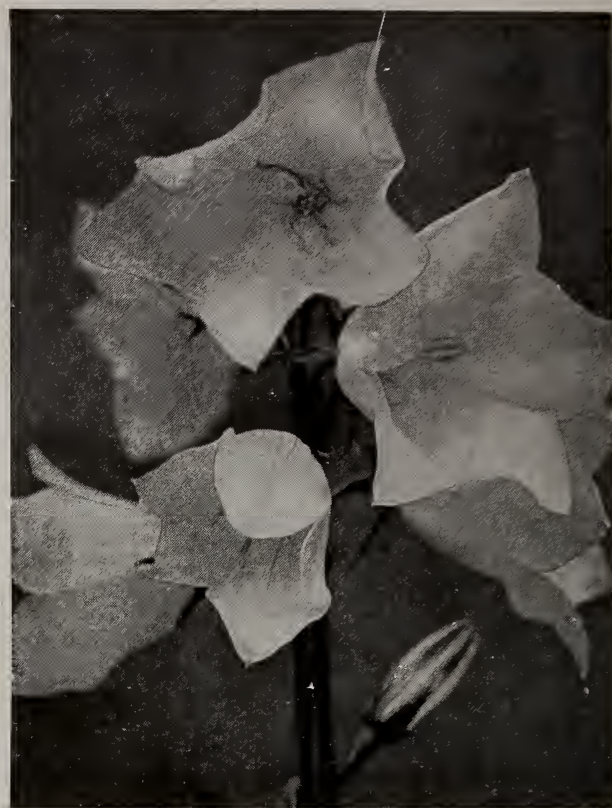

CAMPANULA PERSICIFOLIA

\section{CENTAUREA}

T. pkt.

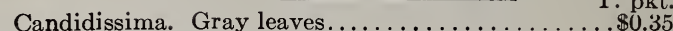

†Gymnocarpa. Wide, white leaves..................... $10 \quad \$ 0.50$

\section{FLOWERING VARIETIES}

Americana. Lilac.......................... $\quad .10 \quad .35$

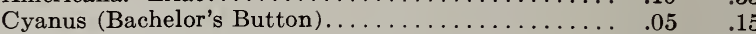

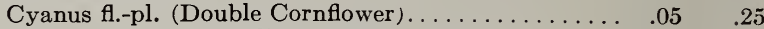

Cyanus, Emperor William. Blue............... .05 $\quad .15$

Cyanus, Victoria. Deep blue; very dwarf............... $\quad .10 \quad .40$

Cyanus, Rose, White..................... . .05

Double Blue Cornflower.................... .15

Imperialis (Sweet Sultan), Mixed...............

Imperialis, White, Yellow................Each. . .10

Imperialis, Lavender..................... . .10

Imperialis moschata rosea......................

Odorata. Large; light blue; sweet-scented........... . .10

Odorata, Margaret. Pure white; fragrant......... .10

Odorata, Chameleon. Yellow and rose............

CASTOR BEANS (Ricinus), Mixed............ .05

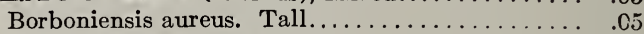

Cambodgensis. Almost black ...............

Gibsoni. Purple stem..................... .05

Sanguinea. Red stem..................... .05

Zanzibarensis. Dark leaves .................

\section{CHR YSANTHEMUM.}

Single. Annual; mixed colors................ .05 .05

Double. Annual; mixed colors................ $.05 \quad .30$

*Bridal Robe. Blooms first year............. . . .15 1.50

†Frutescens (Paris Daisy).................... .10 $\quad .40$

King Edward VII......................... .15

†Shasta Daisy, Alaska ............ 1/8oz. 25c.. $\quad .15$

†Japanese Early-flowering. Mixed............ . .25

†Maximum Perfection. Large; white........... . . . . .

Single Early-flowering Hybrids............. .

†CINERARIA Maritima. White foliage......... .05

Grandiflora. Exceptionally fine quality......... $\quad .50$

Matador. Large, scarlet flowers ............... .35

Nana. Best mixed: dwarf................ $\quad .50$

Radiata. Small flowers; good for cutting....... . .50

CLARKIA, Double Mixed.................... .05

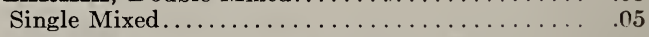

CLEMATIS. Large-flowering Hybrids, Mixed.... . .25

CLIANTHUS Dampieri. Scarlet and black....... .15

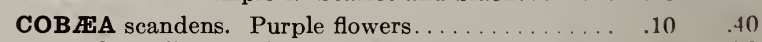

Scandens alba. White flowers............... $\quad .10 \quad .70$

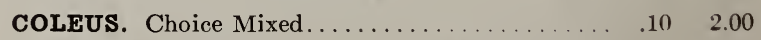


CorvorvUIUS (Morning Glory). Lb. Major. Tall, mixed......... \$0.50

Minor. Dwarf, m.xed.......... .50

Japanese Imperial. Mixed colors $\quad .75$

Japanese Imperial. Best fringed 1.50 See also Ipomoea and Cypress Vine.

Cosmos, early flowering, mixed......\$0.10 $\$ 0.30$

Early Flowering Dawn. White tinged pink .....................10

Early Flowering.

- White, pink, crimson, each......

Giant Flowered Cosmos, mixed........

Giant Flowered Cosmos, in separate colors, red, pink, white, orange, each $\ldots \ldots \ldots \ldots \ldots \ldots \ldots \ldots \ldots \ldots \ldots . \ldots \ldots$

Lady Lenox, very large shell pink

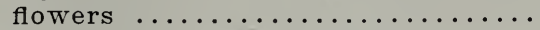

Klondyke. Orange yellow............

Margaret. Large, fringed flowers.... .10

fCowsLIP (Primula Veris) mixed..... .10

CUPHEA Platycentra (Cigar Plant).. .15

\section{Large Flowering CYCLAMEN German Grown}

In separate colors-Dark red, pure white, white with red eye, brilliant rose, lilac, pink. Trade pkt. $15 \mathrm{c} ; 100$ seeds, $60 \mathrm{c} ; 1,000$ seeds, $\$ 5.00$.

All the above mixed: Trade pkt. 15c; 100 seeds, $50 \mathrm{c} ; 1,000$ seeds, $\$ 4.25$.

\section{Special Florists' Strain}

Glowing Dark Red

Rose of Marienthal, rose

Pure White

Trade pkt. 20c; 100 seeds, $\$ 1.00 ; 1,000$ seeds, $\$ 8.00$.

Glory of Wandsbeck, salmon color.

Rose of Wandsbeck, red salmon.

Album Violaceo Striatum, striped.

Trade pkt. $25 \mathrm{c} ; 100$ seeds, $\$ 1.25 ; 1,000$ seeds, $\$ 10.00$.

\section{Giant Flowered, English Grown}

Album, white.

Duke of Fife, dark rose.

Picturatum, light rose, tinted with claret base.

Duke of Connaught, purplish crimson.

Trade pkt. $20 \mathrm{c} ; 100$ seeds, $\$ 1.00 ; 1,000$ seeds, $\$ 8.00$.

\section{Giant Flowered Fancy Cyclamen}

This is a famous strain grown by a Cyclamen specialist in Europe. It brings very handsome plants, with extra-large and very distinct flowers. We consider it one of the very finest strains of Cyclamen seed that has been offered to the trade in a long time, and believe that both plants and flowers will give the greatest satisfaction. We offer mixed seed only. Trade pkt., 20c.; 100 seeds, $\$ 1.00 ; 1,000$ seeds, $\$ 8.00$.

\section{Orchid Flowered or Butterfly}

White, White with Carmine eye, Lilac and Pink, Trade pkt., 20c.; 100 seeds, 85 c.; 1,000 seeds, $\$ 7.00$. Red. Trade pkt., 20c.; 100 seeds, $\$ 1.00 ; 1,000$ seeds, $\$ 8.00$.

CYPFT T. Pkt. Oz.

CY PER.US alternifolius (Umbrella Plant)......\$0.15 \$1.25

Separate colors, Red, White, each.............

DAFIIA, double, extra choice, mixed... $.20 \quad 1.15$

Cactus-flowered, double, mixed............... $20 \quad 1.25$

Single-flowered, mixed .............................

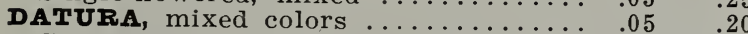

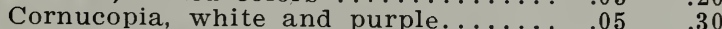

†DIIPHINIUIM (Annual varieties, see Larkspur).

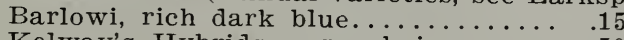

Kelway's Hybrids, very choice............. 50

Formosum Coelestinum, sky blue....

Formosum, dark blue, white center.. $\quad .10$

Nudicaule, orange scarlet.......... .20

Elatum nanum, sky blue to indigo... .20

Belladonna, turquoise blue, fine..... .25

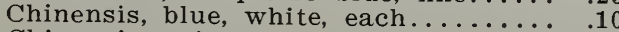

Chinensis, mixed

Gold Medal Hybrids ..............

\section{DIANTHUS}

(Double Pinks.)

Chinensis, mixed, extra fine.......

Chinensis, albus, fl.-pl., white......

Heddewigii fl.-pl., mixed

Diadematus (Diadem Pink)

Laciniatus fl.-pl., fringed, mixed

Laciniatus mirabilis, mixed.

Imperialis fl.-pl. (Imperial Pink)

Double Pinks, all kinds mixed.

T. Pkt. Oz.

$\$ 0.05 \quad \$ 0.35$

.05

.10

.10

.15

.15

.10

.35

Single Annual Pinks

Laciniatus, fringed petals, rich colors

Laciniatus mirabilis, new and fine.

Japan, striped and spotted.

Crimson Belle, blood-red; large flowers

Eastern Queen, light and dark mauve.

The Bride, white, with purple eye.

Mourning Cloak, dark, velvety crimson.

Single Pinks, all colors, mixed.

\section{Hardy Perennial Pinks}

Plumarius semperflorens, double mixed........... .15

Plumarius albus.

Plumarius, single (Pheasant's Eye).

Plumarius, double.

$†$ DIGITALIS (Foxglove) mixed.....

Yellow.

Gloxiniæflora, large flowers, mixed.

Gloxiniæflora, White, Purple, Rose, each

Monstrosa purpurea..

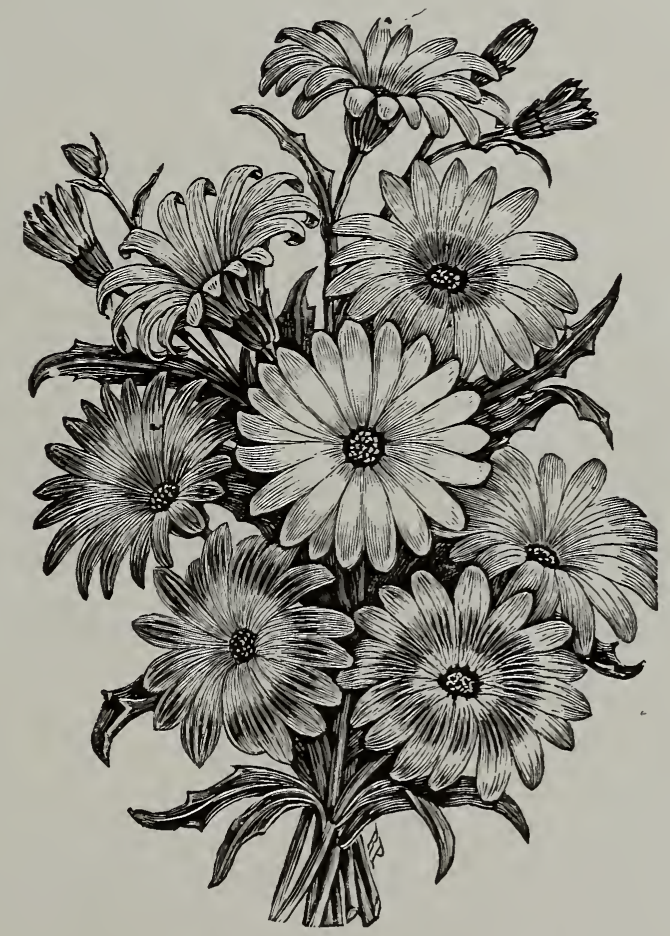

\section{Golden Orange Daisy Hybrids}

(Dimorphotheca aurantiaca hybrida.)

In a general way these hybrids resemble the parent plant but their colors run from purest white to deep orange with varied colored zones. They are most easily grown from seed. When bedded out the brightness of their colors makes them universally admired. Pkt. $20 \mathrm{c}$.

DIMORPHOTHECA (African Golden Daisy), grand bedding plant..........15

DoLICHos (Hyacinth Bean) mixed.... .0 Daylight, pure white.............. .05

DRACAENA Indivisa, narrow foliage.. . .10 Indivisa Lineata ................. .10 Australis, broad leaved.............

ECHINOCYSTIS (Wild Cucumber)..... .05 ECHEVRRIA, Secunda, 1,000 seeds,

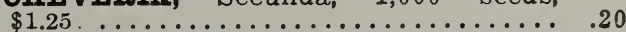




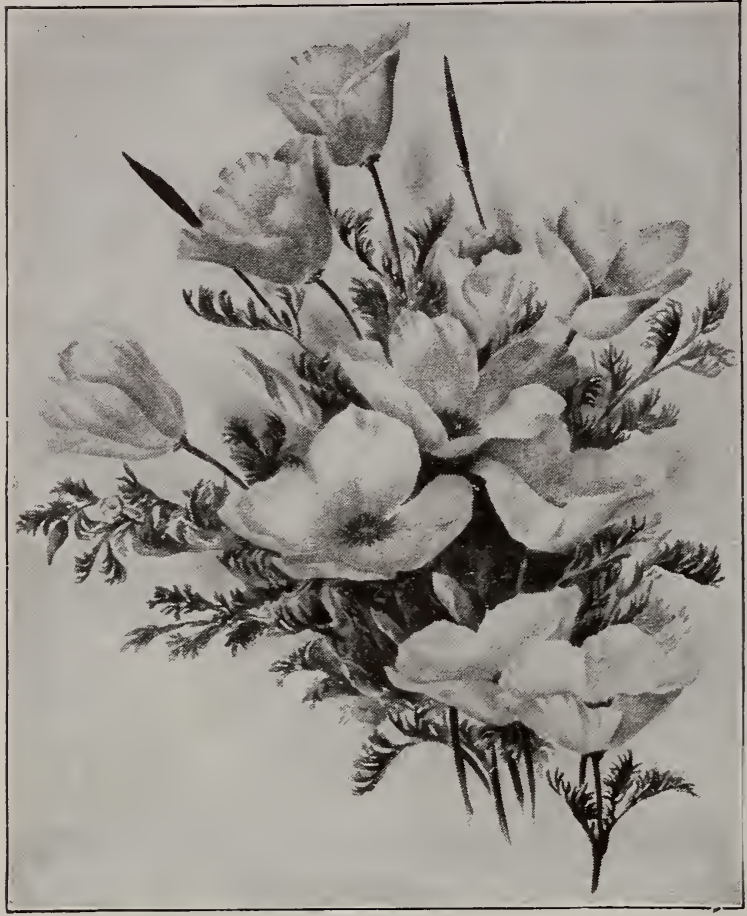

ESCHSCHOLTZIA

\section{ESCHSCHOLTZIA (California Poppy)}

Golden West. Canary-yellow............. ${ }_{\$ 0.05} \mathrm{O}_{\$ 0.20}$

Rose-Cardinal. Dark rose; pretty ................. .05

Bush (Hunnemania). Yellow................. .05

Mandarin. Single; scarlet and orange................

Single. Many colors mixed.................... .0 .05

Double. Many colors mixed................. . .05

FEVERFEW (Matricaria). Double whitc......... .05 $\quad .20$

FUCHSIA. Double mixed................ .25

FOUR-O'CLOCK. Tall or dwarf, mixed....... .05

GAILLARDIA (Blanket Flower).

Lorenziana. Red and Yellow; double......... . .05

Single. Choice varieties; mixed ............. 05

†Kelway's Newest Hybrids. Extra-fine.......... . . . .

†Grandiflora superba. Very large flowers........... .

New Hybrid........................... .10

GERANIUM zonale. Mixed colors........... . 10

Apple-scented. Fragrant................. .25

GILIA. Good cut-flower. Mixed............. .05

Dwarf White. Good for edgings.............. .05

GOMPHRENA (Everlasting). Mixed.

GLOXINIA, Defiance. Scarlet flowers

Splendid Mixture of Beautiful Colors

GODETIA. Fine cut-flower; mixed.

GOURDS. Mixed ornamental varietics.

GREVILLEA robusta. Fern-like foliage

GYPSOPHILA elegans. White, dwarf

Elegans rosea....

Paniculata fl.-pl. Double...

†Paniculata. White......................

HELIANTHUS, Double Purple..

Multiflorus. Double yellow; dwarf. .

Miniature. Single; orange; fine form

Globosus fistulosus. Round; double.

Chrysanthemum-flowered.

HELICHRYSUM (Everlasting). Mixed

Silver Ball... ...

Album.

Fireball.......

HELIOTROPE, Choice Mixed Varieties

Dark Sorts, Mixed.

Mammoth-flowered. Mixed.

HIBISCUS, Crimson Eye...

Golden Bowl...

\section{HOLLYHOCKS}

White.........\$0.10 $\$ 0.75 \quad$ Pink......... T. Pkt. $\begin{aligned} & \text { Oz. } \\ & \$ 0.10\end{aligned}$

Crimson......... $\quad .10 \quad .75$ Yellow........... $\quad .10 \quad .75$

Salmon....... $10 \quad .75$ Naruon......... $\quad .10 \quad .75$

Double Hollyhocks, mixed................... . . . $\quad .60$

Chater's Prize Hollyhocks, Mixed.............. .15

Allegheny Hollyhocks....................... .10

Single Hollyhocks, Mixed................

†HUNNEMANNIA (Bush Eschscholtzia)........ .05

HUMULUS Japonicus (Japan Hop)........... . .05

Japonicus variegatus. Foliage variegated........... .05

ICE PLANT (Mesembryanthemum crystallinum).... $\quad .05$

IMPATIENS Sultana. Carmine.............. . .20

Sultana Hybrids. Various colors............. $\quad .20$

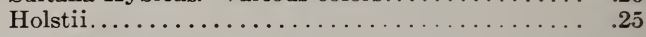

IPOM \&A (Moonflower). White seed $\ldots \ldots \ldots \ldots \ldots, .10 \quad .35$

Black seed..................................

Bona Nox (Evening Glory)....

Pink Hybrids..................... . .10

$\begin{array}{llll}\text { Heavenly Blue. Large sky-blue flowers . . . . . . . . . } \quad .10 & .10\end{array}$

Purpurea. See Convolvulus.

KOCHIA tricophylla (Summer Cypress) ......... . . . $\quad .50$

KUDZU VINE (Jack-and-the-Beanstalk) ........ . .10 $\quad .50$

LANTANA. Finest Mixed................. . . . $\quad .25$

LARKSPUR. Annual varieties.

Tall, Sky-Blue, Dark Blue, Shell-Pink, White.Each. .10

Double Branching. Mixed.................. .05

Emperor. Compact......................

Giant Hyacinth-flowered...

$\dagger$ For Perennial sorts, see Delphinium.

†LATHYRUS latifolius (Hardy Sweet Pea). Red, White,

Pink Beauty......................... Each. .10

Mixed............................ .10

†LAVENDER. Fragrant blue flowers.......... . 05

LEMON VERBENA. Comes readily from seed... . . $\quad .20$

LINARIA Cymbalaria (Kenilworth Ivy)........ . .15

Maroccana "Excelsior" .................... .15

LINUM grandiflorum rubrum (Scarlet I lax)..... . . . 05

†Perennial, Mixed...................... .05

LOBELIA alba. White................ .10

Sapphire. Deep blue; pendent............... $\quad .20$

Speciosa. Dark foliage; blue............... .10

Barnard's Perpetual. Blue, white eye; dwarf; for

edgings and baskets.........................

Bedding Queen. Purple, with white eye; dwarf... $1 / 40 \mathrm{Oz} .50 \mathrm{c} . . .15$

Crystal Palace Compacta. Dark blue............. .15

Emperor William. Blue; dwarf .............. .10

Prima Donna. Rich velvety crimson.......... $\quad .15$

White Gem. Dwarf; fine pot plant........... . 10

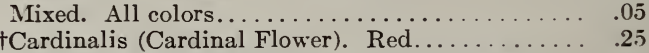

†Queen Victoria. Red foliage; scarlet flowers..... . . .

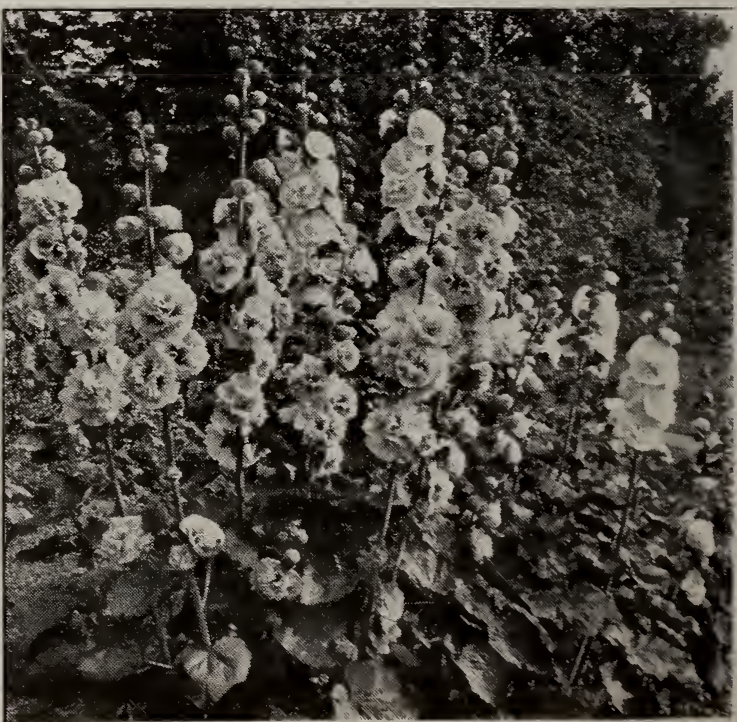

HOLLYHOCKS 


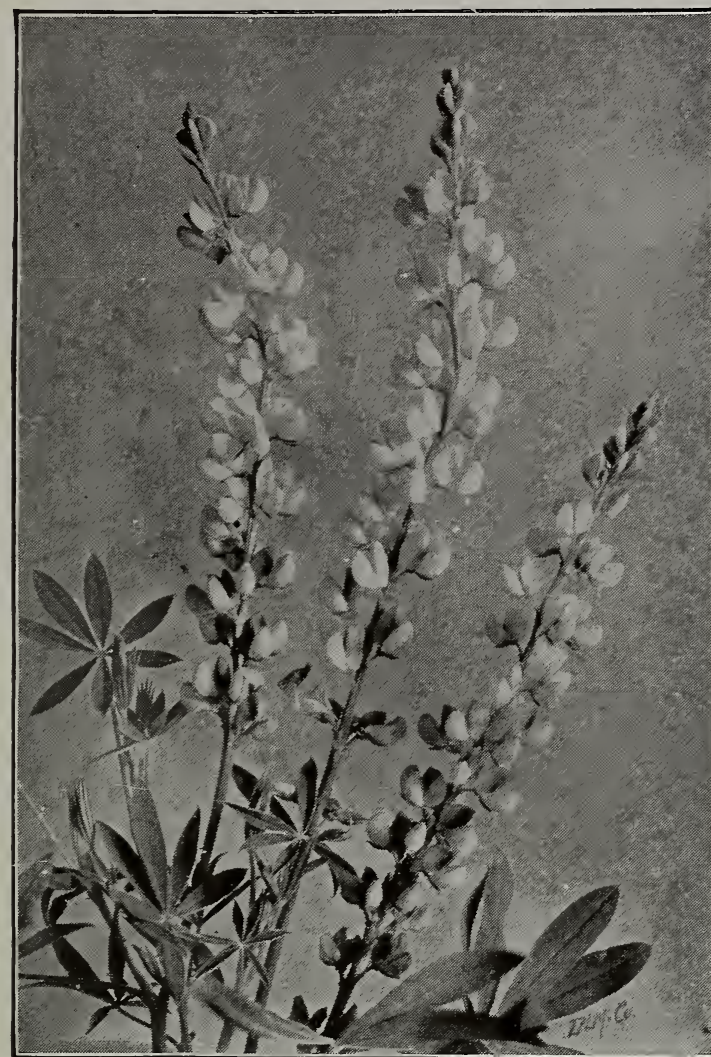

LUPINUS POLYPHYLLUS

LOPHOSPERMUM scandens. Climbing.. $\begin{array}{lr}\text { T. pkt. } & \mathrm{Oz} . \\ . . \$ 0.15 & \$ 1.50\end{array}$

LUPINUS. Tall Annual Sorts. Mixed. .

Dwarf Annual Sorts. Mixed...................

Annual. White......................

Hybridus roseus. Pink.................. .05

Hybridus cæruleus. Blue................. .05

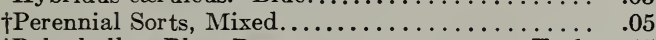

†Polyphyllus, Blue, Rose ..................... .15

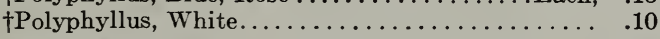

†LYCHNIS Chalcedonica. Scarlet............ 05

†Haageana hybrida. Mixed................. .15

MARIGOLD, African. Double. Mixed. Tall..... .05

El Dorado. Yellow to orange; tall............ .05

Lemon Queen. Extra-fine strain; tall......... .05

Double Dwarf French. Mixed ................. .05

Dwarf Legion of Honor. Yellow and brown...... .05

Double Dwarf. Gold-striped................ . .05

MATRICARIA Capensis (Feverfew) .........

Golden Ball......................... .15

MAURANDIA, Mixed.................. 10

MIGNONETTE, Allen's Defiance. Long, full spikes. $\quad .10$

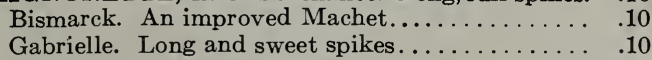

Grandiflora. Large-flowering. . . . . . . . . . . . .05

Goliath. Red; large blooms ................ $\quad .20$

Golden Queen. A garden variety .............. .05

Machet. Long spikes of red flowers.......... . .10

Golden Machet. Best French strain............ .10

New York Market. For greenhouses.......... . .35

MIMOSA pudica (Sensitive Plant) .......... .05

MIMULUS tigrinus (Monkey Flower) . . . . . . . .15

Moschatus (Musk Plant) ..........1/80z. 20c... .10

MOMORDICA Balsamina (Balsam Apple)....... . .05

Charantia (Balsam Pear) .................. .05

MOONFLOWER. See Ipomœa.

f MUSA Ensete........100 seeds, 85c.; 15 seeds, 20c. . .

MYOSOTIS (Forget-Me-Not). Mixed.......... .05

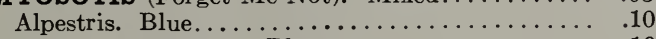

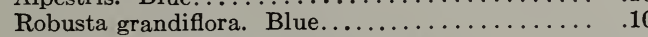

Victoria. Sky-blue; good for cutting............ .15

Dissitiflora. Clear blue; blooms early ........... .25

Palustris (True Forget-Me-Not). Dark...............

Ruth Fischer. Large; blue............... .25

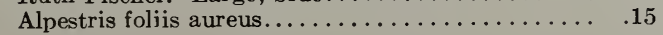

\section{NASTURTIUM}

Besides their ordinary garden use for trailing over fences, trellises, stone walls, etc., nasturtiums can also be grown as potplants for winter flowering, as screens, or as trailers for hangingbaskets and vases.

\section{TALL OR CLIMBING}

Atropurpureum. Velvety crimson...

Oz. $\quad 1 / 4 \mathrm{lb}$

Chameleon. Different colors on one plant.......... $.10 \quad .25$

Coccineum. Bright scarlet.................. .10 .15

Dunnett's Orange. Orange and red.............. $\quad .10 \quad .15$

Edward Otto. Light brown; yellow spur.......... $\quad .10 \quad .15$

Heinemanni. Chocolate color................ $\quad .10 \quad .15$

Hemisphæricum. Orange; handsome............ . .10 $\quad .15$

King Theodore. Crimson; dark foliage............ $\quad .10 \quad .25$

Pearl. Creamy white..................... $\quad .10 \quad .15$

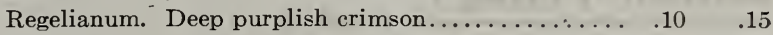

Schillingii. Yellow and red................... .10 $\quad .15$

Scheuerianum. Straw-color; spotted............. . .10 $\quad .15$

Sunlight. Rich golden yellow; a gem........... $\quad .10 \quad .30$

Von Moltke. Rose........................ .10 .15

Barnard's Special Mixture of Tall Nasturtiums, made

up from named sorts................Lb. 75c... $\quad .10 \quad .20$

Madam Gunther Hybrids. Dark foliage. Lb. 75c. . $\quad .10 \quad .20$

Tall, Mixed. Excellent value.............Lb. 45c.. . .05 $\quad .15$

\section{DWARF OR TOM THUMB}

Splendid bedders; bloom in two months from seed. Aurora. Salmon and rose-blush.....

Beauty. Scarlet, splashed canary.

Cloth of Gold. Scarlet; yellow foliage............. 10

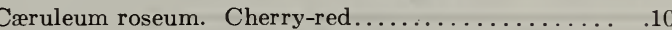

Crystal Palace Gem. Sulphur-yellow.......... . .10

Empress of India. Crimson; dark leaves. . . . . . . . . .10

Golden King. Yellow; dark foliage............ .10

King of Tom Thumbs. Searlet; dark.......... .10

King Theodore. Maroon; dark leaves............ . $\quad .10$

Lady Bird. Yellow and red................. .10

Pearl. Creamy white..................... .10

Prince Henry. Cream, marbled scarlet........... . 10

Rose. Soft, pleasing color ................. .10

Ruby King. Ruby-rose; dark.......................
Variegated-leaved. Red flowers. Scarlet, Spotted,

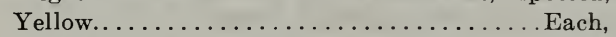

\section{.20}

.25

.25

.30

.25

.25

.35
.20

1.25

.25

.60

.50

.15

.40

.50

.50

.25

1.00

1.25

30

.30
Barnard's Special Mixture of Dwarf Nasturtiums,

made up from named sorts...........Lb. 75c... .10

Dwarf Mixed. A fine assortment..........Lb. 60c.. . .05

.20

.20

.20

.20

.20

.20

.20

.20

.20

.20

.20

.20

.20

20

.20

\section{LOBB'S CLIMBING}

Cardinal. Dark cardinal-red; superb............ $\$ 0.10 \quad \begin{aligned} & 1 / 4 \mathrm{lb} . \\ & \$ 0.20\end{aligned}$

Giant of Battles. Sulphur, spotted with red........ $\quad .10 \quad .20$

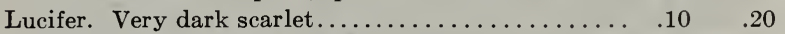

Napoleon III. Golden striped carmine........... $\quad .10 \quad .20$

King of the Blacks. Black-brown................ $\quad .10 \quad ._{20}$

Spitfire. Brilliant scarlet................... . $10 \quad .20$

Mixed, All Colors..................... $10 \ldots \ldots$

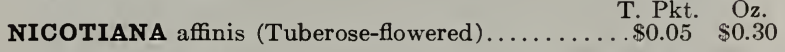

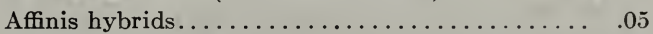

Sanderea. Carmine-red................. . 10

Hybrids. Collection of 8 colors . . . . . . . . . . 15

Sylvestris. White flowers................. 05

NIEREMBERGIA gracilis. Blue........... .10

†NIGELLA. Good for cut-flowers. Mixed....... .05

PANSY. See page 8.

PASSIFLORA (Passion Flower).

PENNISETUM (Fountain Grass).

Longistylum. Grayish plumes.

Ruppelianum. (Purple Feather Grass).

PENTSTEMON, Large-flowered. Mixed.

Hartwegi. Creamy white flowers......

PEPPER. Branching for pots ....... 1/80z. 35c.. .20

Cluster for pots............... $1 / 8 \mathrm{oz} .35 \mathrm{c} \ldots \quad .20$

Celestial. Xmas variety........1/80z. 45c... .25 


\section{Pansies Various Named and in Mixtures.}

All the Under Mentioned Varieties Can Be Relied Upon as Being True to Description and Color.

Psyche. Veiy fioriferous and perfect in habit. Each S Hower is elegantly curled and undulateu and in every respect a beauty in form and color. The characteristical velvety violet spots or eyes are surrounded with a broad white edge. Comes true from seed. Trade pkt., 25c. Hero. A fine bedding variety. Crumpled and curled white. Trade pkt., 25c.

"Masterpiece." The nearest apprcach yet curled and twisted. Very large flowers more or less blotched and in combinations of color. Trade pkt., 25c.

MADAM PERRET. Flowers from 3 to $3 \frac{1}{2}$ inches in through all the shades of red, from pink to an occasional bright red edged with white. Trade pkt., 15c.

\section{Florists' Pansy Mixture.}

$W^{E}$ can best describe this as a combination of seeds of the best named Pansies, secured by us from leading specialists both at home and abroad. In the many years we have supplied this "FLORISTS" MIXTURE," so far as we are aware, it has never failed to prove satisfactory. Our aim is to furnish the best obtainable and to that end no expense is spared. Trade pit., 25c ; 1/8 oz., \$1; 1/2 oz., \$3.50 ; oz., \$7.

\section{Giant Flowered Pansies.}

It is poor economy to expect the best results from any other than high grade seeds. We have secured an extra fine quality of Giant Pansy Seed from which, we feel certain, most satisfactory flowers will be obtained.

Trade pkt. $1 / 8$ oz. oz.

Giant Auricula colors, dark and velvety. $\begin{array}{llll}\$ 0.10 & \$ 0.20 & \$ 1.25\end{array}$

Giant Aurora, pure white................ $10 \quad .25 \quad 1.50$

Giant Azure blue, beautiful shade..........10 $\quad .25 \quad 1.25$

Giant Bugnot, a splendid strain, having

large, round flowers richly marked, unex-

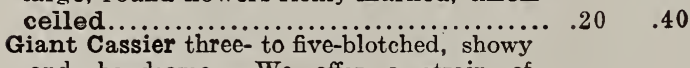

and handsome. We offer a strain of superior quality.......................

Giant Coal Black, a fine, very dark variety.

Giant Fiery Faces, scarlet, gold edge, yellow center...

Giant Five-spotted, petals marked and

blotched; superb................................ .25

Giant Freya, purple, margined with white...
Giant Lord Beaconsfield, lavender, violet and purple...

$.15 \quad .35$

$\begin{array}{lll}.10 & .25 & 1.50\end{array}$

$\begin{array}{lll}.10 & .20 & 1.25\end{array}$

Mr. Troth, Van Buren Co., Iowa, writes: "The of Pansy seed has germinated in grand shape. I do not think any failed to grow."

Mrs. O. M. W., Cook Co., Ill., writes: "We had such wonderful results from the Pansy seeds bought from you last year that we are encouraged to try, more of your line. We had hundreds and hundreds of blossoms."

\section{PRIZE PANSIES.}

Unequalled for Bedding Out.

$1 / 40 z$, oz. Azure Blue......... \$0.25 $\$ 0.80$

Black ( $F$ a us t's

King of Blacks). $.25 \quad .90$

Black and crimson. $.25 \quad .80$

Bronze ..........30 1.00

Brown shades .....25 $\quad .80$

Candidissima.......30 1.00

Cardinal, scarlet.. .50 1.20

\section{1/4 oz. Oz.}

Dark Purple,rich. $\$ 0.25 \$ 0.80$

$\begin{array}{lll}\text { Emperor Frederick. } & .25 & .80\end{array}$

Emp. Wm., ultra-

marine ............ $30 \quad 1.00$

English Face..... $.25 \quad .80$

Fire King, yellow. $\quad .25 \quad .80$

Golden Gem, pure

yellow........... $30 \quad 1.00$

PRIZE PANSIES, mixed, all the above and others in proper ocnmortion of colors. Trade pkt., 10c : $1 / 4$ oz.. 30c; oz., $\$ 1.00$
FAIRY QUEEN. Sky blue, margined with silvery white. Trade pkt., 10c; $1 / 8$ oz., 30c; oz., $\$ 1.00$.

HORTENSIA. Light and dark rose-colored, blotched flowe $s$ of giant size. Trade pkt., $20 \mathrm{c} ; 1 / 8 \mathrm{oz} ., 50 \mathrm{c}$.

COQUETTE DE POISSY。 Slate or mouse-colored flowers of gord size and form. Trade pkt., 10c; $1 / 8$ oz., 20c: oz., $\$ 1.00$

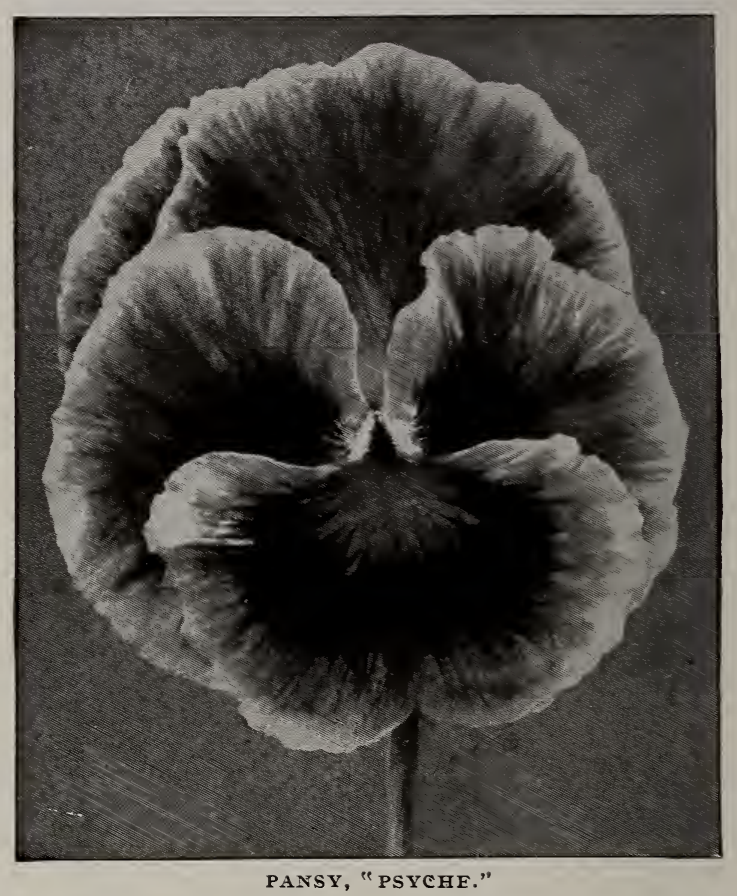

Trade pkt., $1 / 8$ oz. oz,

Giant Odier, a showy and richly colored strain

with very large blotched flowers..........\$0.20 \$0.35 \$2.50

$\begin{array}{lllll}\text { Giant Parisian, large, striped flowers......... } & .10 & .20 & 1.50\end{array}$

Giant President Carnot, violet blotch on white ground.

$\begin{array}{lll}.15 & .30 & 2.00\end{array}$

Giant Purple, rich and velvety........... $\quad .10 \quad .20 \quad 1.25$

Giant Snowflake, pure white............. $.10 \quad .25 \quad 1.50$

$\begin{array}{llll}\text { Giant Spotted White, white, dark blotch.... } & .10 & .20 & 1.25\end{array}$

Giant Striped, brightly striped and streaked.. $\quad .10 \quad .25 \quad 1.50$

Giant Yellow, dark center................20 $\quad .25 \quad 1.25$

Giant Yellow Gem, clear yellow........... $.10 \quad .25 \quad 1.50$

Giant Violet Blue, a pleasing combination $\begin{array}{llll}.10 & .20 & 1.25\end{array}$

Giant Trimardeau, Improved, remark-

able for the immense size of the flowers,

most of which are blotched and spotted.... $\quad .10 \quad .20 \quad 1.00$

Giant Mixture Includes seeds of all the Gian Gant Mixture Pansies named above, as well as other very choice sorts not otherwise obtainable Trade pkt., 20c; 1/8 uz., 50c; 1/2 oz., \$2.00; oz., \$4.00.

Finest imported German seed of any of the following PRICE: Trade pkt., 10c.

I/4 oz. oz.

I/t oz. oz

Gold Margined...... \$0.25 $\$ 0.90$, Prince Bismarck, $.30 \$ 1.00$

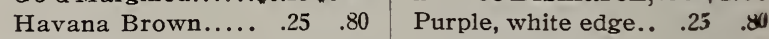
$\begin{array}{lllllll}\text { Light Blue.......... } & .25 & .90 & \text { Quadricolor......... } & .25 & .80\end{array}$ Lord Beaconsfield .30 1.00 Silver Edge, dark Mahogany Colors.. $.301 .00 \quad$ purple, edged $\begin{array}{llllll}\text { Mottled and striped. } & .35 & 1.20 \quad \text { white.............. } & .25 & .80\end{array}$ Pe a c o c k, blue,

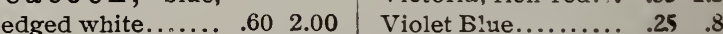

PANSY SEED. - A good mixture of many colors and shades. Oz., 50c: 5 oz. for $\$ 2.00$ 


\section{PETUNIAS}

A $p \circ r-$ tion of the see d 1 in $\mathrm{g}$ s a $\mathrm{re}$ liable to $\mathrm{prove}$ semi-double. The weake st plants gene $\mathrm{r}$ a $11 \mathrm{y}$ produce the finest double fl o $\mathrm{w}$ e $\mathrm{r}$. These $\mathrm{re}$ marks also a p ply to $t h$ e single flow e $r$ i $\mathrm{g}$. sort.

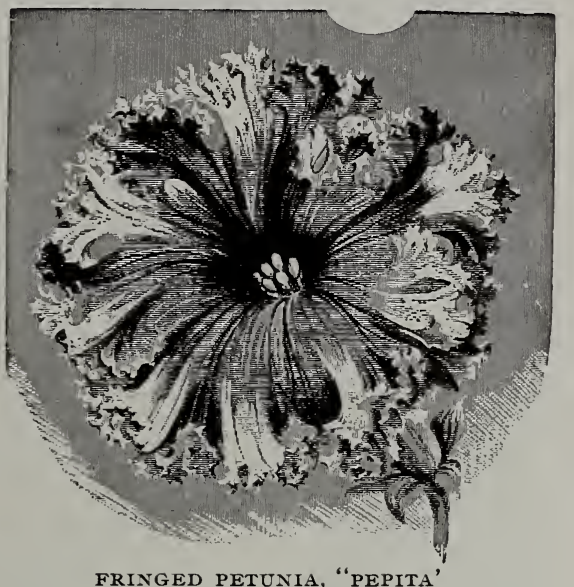

T. Pkt.

Single, fine mixed. z. 50 c...\$0.05

Alba, white....

Inimitable, dwarf, for pots.

Blotched and striped. .

Rosy Morn.

Snowball, fine for pots and beds.

Adonis, carmine

Howard's Star, dark maroon.

Large-flowering, "Giants of California".

Grandiflora fimbriata, fringed.

Grandiflora quadricolor, rose with yellow

Grandiflora, splendid flowers, mixed.

Canary Bird, fringed, clear yellow.

Pepita, deep carmine bordered white

Violet-Blue, fine bedder.

Ruffled Giants.

Dwarf large-flowering fringed mixed.

DOUBLE

Fringed, large, fine flowers.

Pure White (Lily of the Lake); the very best

Barnard's Double Mixed Petunias.

\section{PHLOX Drummondi, Grandiflora}

White $\ldots \ldots . \$ 0.10 \quad \$ 0.75$ Soft Pink...\$ T. Pkt. Oz. Scarlet … $.10 \quad .75$ Carmine, white Rosy Carmine $.10 \quad .75$ eye ........ Best large flowering, all colors, mixed. $\quad .10$

PHLOX DRUMMONDI, fine mixed..... .10

Star of Quedlinburg (Star Phlox)

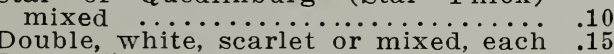

Double, white, scarlet or mixed, each best ..........................15

Dwrxf Sorts-Nana Compacts. T.Pkt. $1 / 4$ oz.

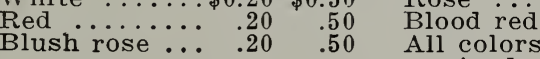
T.Pkt. $1 / 4$ oz $\$ 0.20 \quad \$ 0.50$

†Decussata (Hardy Pnlox) mixed...... Oz. $\$ 1.25$. .15

tDecussata, White, Rose, Red, Carmine with

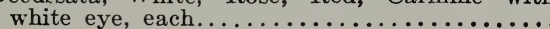

†PLATYCODON (Chinese Bellflower),

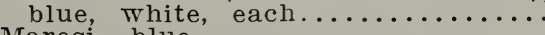

Maresi, blue ................. 10

\section{POPPY (Papaver)}

Poppy, single, mixed, annual............ 05

King Edward, scarlet, shaded black.. .10

Burbank, improved Shirley ..

Shiriey, a favorite for cut flowers.. .05

Tulip, brilliant scarlet............ 10

Double, mixed, annual..........................

Cardinal, scarlet

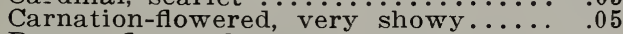

Paeony-flowered, mixed colors........... .05

White Swan, large, pure white..... .05

†udicaule (Iceland), mixed colors . ............

TOrange, White, Scarlet, each $\ldots \ldots \ldots . . . . .10$

tOrientale, crimson; black blotch............... .10

tOrientale, new hybrids ......................

PORTULACA, single, finest mixed.

Double, extra fine mixed...................

\section{Primula Sinensis Fimbriata}

Finest, English grown. The flowers are not only very large in size, but have excellent substance, making them lasting. We can supply in a variety of colors and also in mixtures.

Any of the following at 50e per trade pkt. SINGLE FRINGED VARIETIES.

Chiswick Red, bright Filicifolia (Fern leaved), crimson scarlet.

Crimson King, rich color; Gigantea (Giant flowdark brown eye. ered) mixed.

Duke of York, crimson.

Rose Queen.

Urange King.

Ruby Queen.

Snowden, large, white.

Barnard's Mixed. Made

up from named sorts.

Rosea delicata, blush

His Majesty, crimson.

Queen Alexander, white very large flowers.

True Blue.

Magnifica Blue, light

About June 1st of each year we issue a special circular of Calceolaria, Cineraria, Primula and other seeds for florists. Also biennials and perennials for summer

\section{Primula Obconica Grandiflora}

This strain is a very great improvement over the old Obconica. Some of the flowers are almost as large as the Chinese Primroses. Grandiflora alba, pure white..........\$0.50 Oculata ........\$0.50|Rosea, pink ...... .50 Apple blossom......... .50|Kermesina, crimson.. $\quad .50$

Fimbriata rosea, fringed, rose........... $\mathbf{. 5 0}$

" mixed colors................ .25

Obconica grandiflora, mixed............. 25 gigantea, finest mixed........... .50

Primula Kewersis, Giant Buttercup........... gigantea kermesina $\ldots \ldots \ldots \ldots \ldots \ldots . .50$

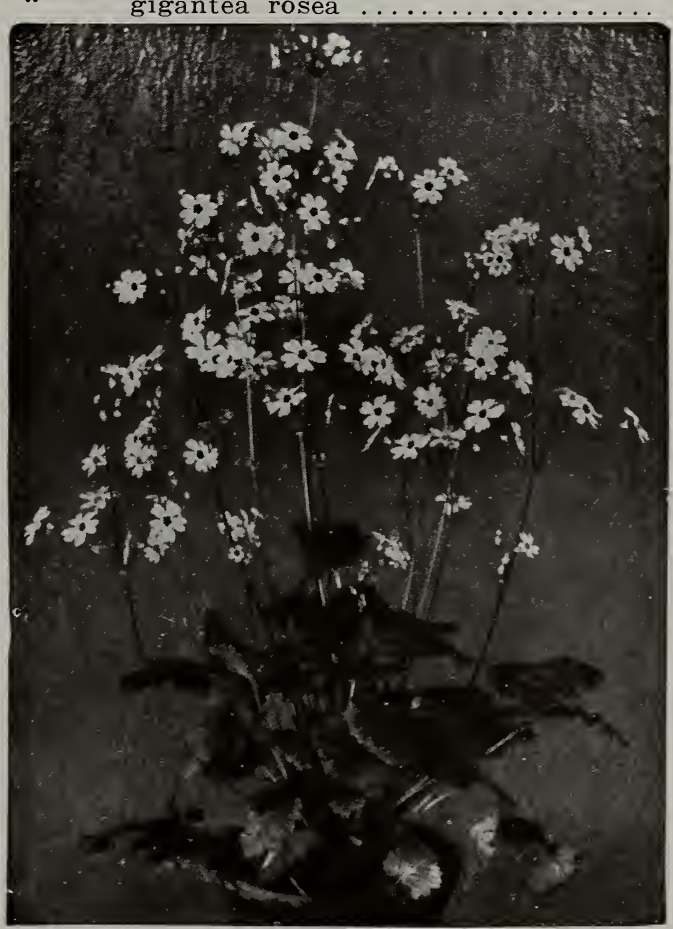

Primula Malacoides

This new variety resembles in general $P$. For besi, but its blooms are larger and plants branch more freely. The light lilac flowers are borne on long stems. Trade Pkt., 25c.

VARIOUS PRIMULAS.

$\dagger$ †uricula, finest mixed..............\$0.25

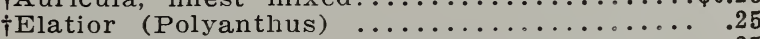

Forbesi (Baby Primrose) mixed.............25

†Japonica (Japan Primrose), mixed.......... .25

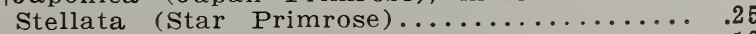

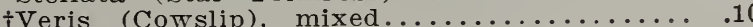

†Vulgaris (English Yellow Primrose)....... .15 
†PUERARIA (Kudzu Vine). Purple.......... T. Pkt. 10 Oz. †PYRETHRUM (Feverfew).

Comet-flowered....................... 20

Aureum (Golden Feather) $\ldots \ldots \ldots \ldots \ldots \ldots \ldots \ldots \ldots \ldots \ldots \ldots, 05$

Giant-flowered Roseum................. 15

Double-flowered Roseum. Mixed.............. 25

Kelway's Hybrids. Very choice strain........ 50

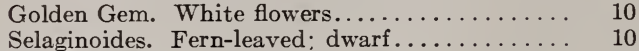

RHODANTHE (Everlasting). Mixed......... 10

RICINUS. See Castor-Oil Bean.

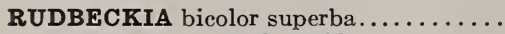

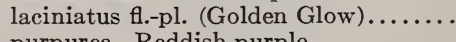

purpurea. Reddish purple...............

SALPIGLOSSIS. Mixed.

Emperor. Large flowers. Mixed.

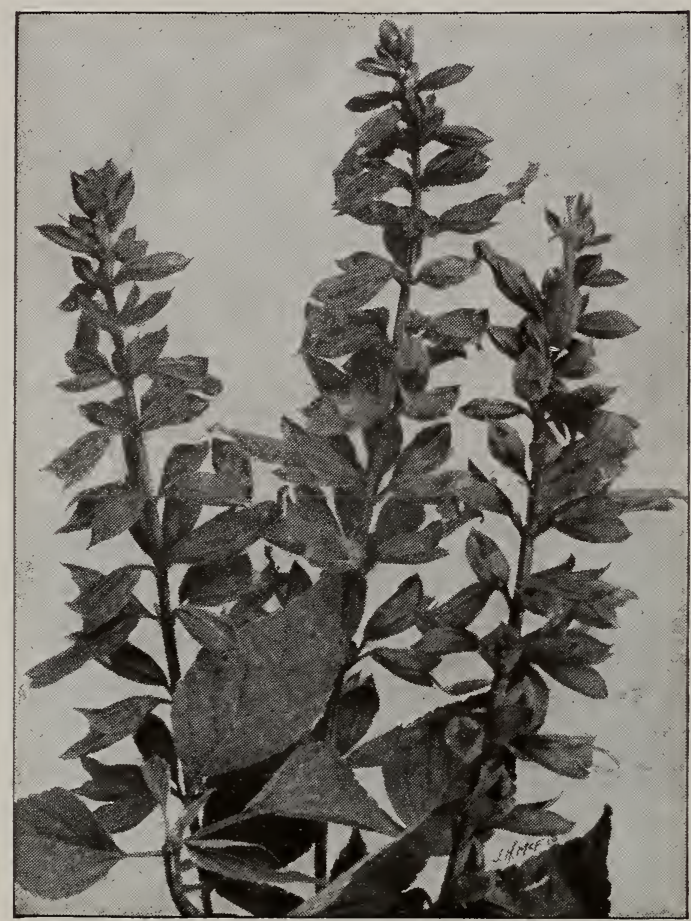

SALVIA SPLENDENS

\section{SALVIA - Scarlet Sage}

Fireball. Brilliant scarlet flowers on erect spikes; T. Pkt. blooms early and continuously............\$ 25

Zurich. Grows only 1 foot high; of same color as Fireball.................. 1/40z. $\$ 1$.

King of the Carpet. Height 18 inches; perfectly round, covering itself with long scarlet flowers down to the ground.

*Globosa. Silver-gray, woolly leaves; white flowers.

Splendens. Bright searlet.

Splendens, Clara Bedman. Scarlet.

Splendens. Drooping spikes; red.

Splendens, St. Louis. Scarlet....

Splendens, Silver Spot. Rich green leaves spotted yellow; flowers scarlet.

$\dagger$ Argentea. Silvery white foliage.

†Patens. Sky-blue.

$1 / 8 \mathrm{oz} .65 \mathrm{c} .$.

SCABIOSA. Tall; double. Mixed

Dwarf double. Mixed

Dwarf Yellow, Pink, Scarlet . .

Snowball. White; 18 inches.

Caucasica. Lavender; 2 to $3 \mathrm{ft}$. .

35

SCHIZANTHUS (Butterfly Flower)

Grahami niveus. Snow-white. ...

Wisetoniensis. Fine for pots....

Large-flowering. Mixed.....
SHAMROCK, Irish.......

T. Pkt. Oz

SILENE pendula. Mixed

SMILAX. New-crop seed..

SOLANUM Capsicastrum.

STEVIA serrata. An excellent cut-flower so 10

05

$10 \$ 0 \quad 25$

$10 \quad 25$

1080

\section{STOCKS, Dwarf Ten Weeks}

German-grown seed saved from flowers of highest quality. Cheap stock seed is a bad investment.

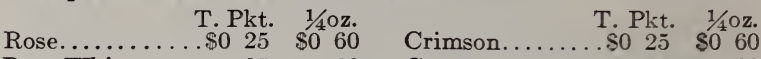

Pure White..... $25 \quad 60$ Canary.......... $25 \quad 60$

$\begin{array}{llllll}\text { Light Blue....... } & 25 & 60 & \text { Purple.......... } & 25 & 60 \\ \text { Blood-Red...... } & 25 & 60 & \text { Mixed........ } & 25 & 50\end{array}$

One packet each seven separate colors, $\$ 1.50$.

\section{VARIOUS STOCKS}

Cut-and-Come-Again (Giant Perfection). This is a valuable variety for cutting; every shoot produces a large spike of double flowers. Ours is the extra giant strain.

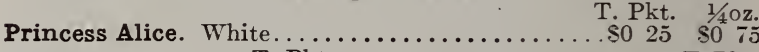

T. Pkt.

Light Blue........... \$0 25 Scarlet............ 025

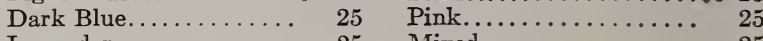

Lavender.............................. 25 Mixed.......... 25

Early Flowering "Nice" Stocks.

Abundance. Flesh-pink.................... 25

Beauıy of Nice. Daybreak-pink..........1/80z.40c... 25

Crim on King. Large flowers.................. 25

Queen Alexandra. Rosy lilac............. 1 \%

Prince Bismarck. A splendid sort for cutting; its pure white flowers are borne on a long spike with many side shoots..........................1/8oz. 40c...

Winter Stock, "White Lady." Blooms earlier and longer than other winter Stocks. A large percentage will come

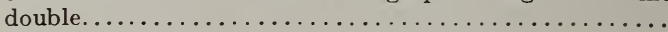

\section{SWEET PEAS, Spencer Type}

This type of Sweet Peas is very largely taking the place of the common or grandiflora sorts. The flowers are larger, often measuring $2 \frac{1}{2}$ inches across. Their beautiful waved petals are borne in clusters of 3 or 4 on long, strong stems, adding so much to their value as cut-flowers. For the past several seasons this type could only be had in a few shades and colors, but now, through careful and persistent work of Sweet Pea specialists, we are able to offer the Spencer type in nearly every shade. The varieties that we are listing here are those which we consider the best of their color, and include some of very recent introduction and of special merit.

America. White striped crimson............. Oz 15 so 55

Asta Ohn. Lavender.................... 15

Blanche Ferry. Pink and white............. 15

Apple Blossom. Bright pink and rose........ $15 \quad 55$

Captain of Blues. Violet-blue............. $15 \quad 55$

Countess Spencer. Delicate pink............ $15 \quad 55$

Dobbie's Cream. (New.) Iarge, handsome flower.

The best of its color.................. $25 \quad 75$

100 Edith Taylor. (New.) Salmon-rose; mammoth

flower........................... $25 \quad 75$

Florence Nightingale. (New.) Delicate lavender. $20 \quad 75$

Flora Norton. Light blue................ $20 \quad 75$

Helen Lewis. Orange-pink................ $20 \quad 75$

25 King Edward VII. Scarlet................ 15 . 55

King White. Very large............... $35 \quad 125$

Margaret Atlee. Delicate pink............. $35 \quad 125$

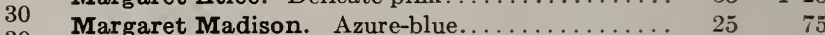

30 Marie Corelli. Bright crimson............. 25

Nubian. (New.) Deep maroon; grand flower..... $25 \quad 75$

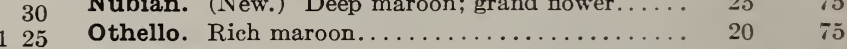

Scarlet Emperor. (New.) Fiery scarlet; splendid. $25 \quad 75$

Stirling Stent. Salmon-pink.............. $25 \quad 75$

Vermilion Brilliant. Bright scarlet.......... 25 75

15 White Spencer. Pure white............... 15 . 55

We pay postage on seeds in packets, ounces and $1 / 4 \mathrm{lbs}$. On 0525 larger quantities please send stamps for parcel postage. 


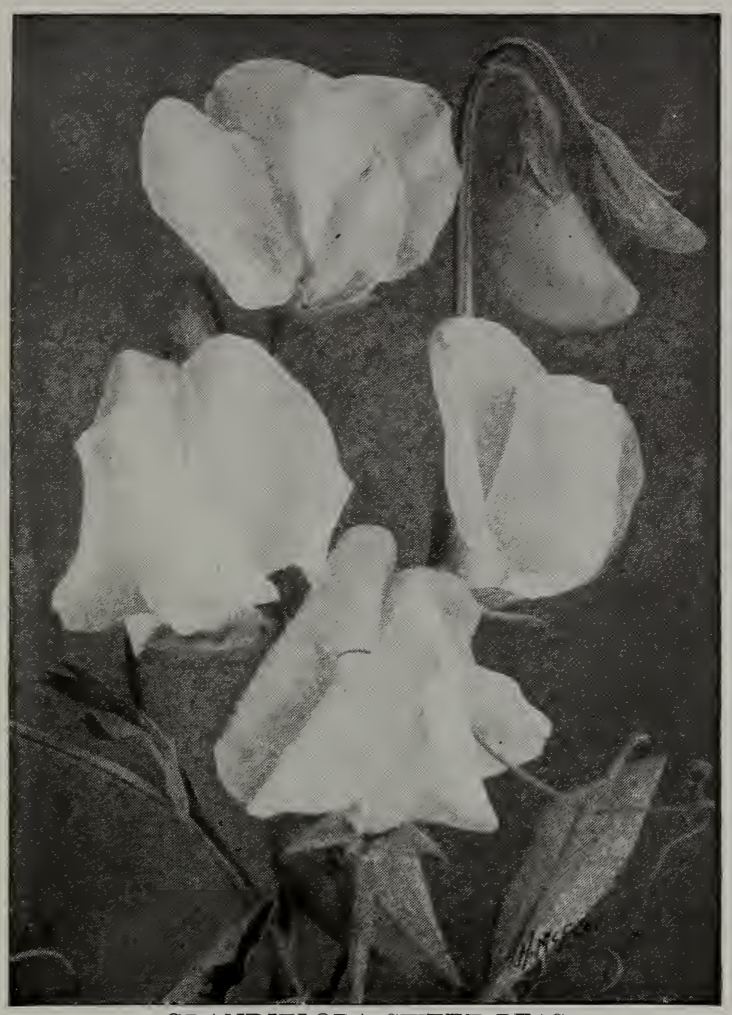

GRANDIFLORA SWEET PEAS

\section{SWEET PEAS, GRANDIFLORA}

To 1 b. price add 8 to $12 \mathrm{cts}$. for parcel post if we are to mail

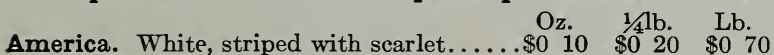
Apple Blossom. Bright rose and pink..... $10 \quad 20 \quad 70$ Black Knight. Dark maroon............ $10 \quad 20 \quad 70$ Blanche Ferry. Pink and white........... $10 \quad 20 \quad 70$ Dorothy Eckford. Best white to date.... $10 \quad 20 \quad 70$ Hon. Mrs. Kenyon. Very fine yellow..... $10 \quad 20 \quad 70$ Katherine Tracy. A lovely pink .......... $10 \quad 20 \quad 70$ Lady Grisel Hamilton. Lavender........ $10 \quad 20 \quad 70$ Lottie Eckford. White, edged blue......... $10 \quad 20 \quad 70$ Miss Willmott. Orange-pink............ $10 \quad 20 \quad 70$ Mrs. Joseph Chamberlain. White on rose. $\begin{array}{llll}10 & 20 & 70\end{array}$ Navy Blue. Deep violet-blue............ 10 Queen Alexandra. Fine scarlet......... 10
†STATICE latifolia. Lavender. T. Pkt. †STOKESIA cyanea. Lavender-blue.

†SWEET ROCKET. White, Purple.

Mixed.

†SWEET SULTAN. See also Centaurea.

SWEET WILLIAM. Single. Mixed.

Double, Giant-flowered Strain.....

Hunt's Perfection.

THUNBERGIA. Fine climber. Mixed....

Yellow, with eye........................

White, with dark eye..

TORENIA Fournieri. Sky-blue $\ldots \ldots \ldots \ldots \ldots$

Fournieri compacta. Porcelain-blue

Nymph. Light blue. .

TRITOMA grandiflora. Hardy .............. 15

\section{VERBENA, MAMMOTH}

T. pkt. Oz. $\quad$ T. pkt. Oz.

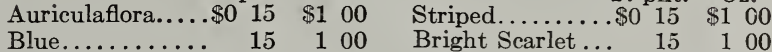

Purple......... $15 \quad 100$ Pure White.... $15 \quad 100$

Pink...................... $15 \quad 15 \quad 15 \quad 100$

Dwarf bedding Verbena. 6 inches high............ $15 \quad 100$

Lemon Verbena. Fragrant leaves........... 20

VINCA rosea. Fine shade of rose............. 10

Rosea alba. White with rose eye............... 10

Rosea alba pura. Pure white............... 10

Mixed colors........................... 10

VIOLA. Single Blue...................... 10

Single White.......................... 10

VIOLA cornuta (Tufted Pansies). Mixed........ 10

Blue Perfection ......................... 15

White Perfection..................... 15

WALLFLOWER Kewensis. Black-brown....... 25

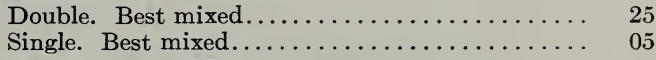

WILD CUCUMBER (Echinocystis) ........... 05

ZEA Japonica (Variegated Maize)............. 05

ZINNIA, Giant-flowered. Mixed............... 10

Tall sorts, in separate colors, White, Yellow, Scarlet, Pink .................... each. 15

Dwarf, Double Mixed Colors............... 10

Crested and Curled........................ 10

Zebra or Carnation-striped...................... 10

Double Mexican. Mixed................... 10

Tall Double. Mixed..................... 10

\section{SWEET PEAS FOR FORCING}

For Christmas flowering sow about the middle of August. Le Marquis. Navy-blue................. $\$ 010$ Burpee's Earliest. White........................ 10

Xmas White...................... 10

Xmas Pink. Pink and white.................. 10

Florence Denzer. White .................. 10

Flamingo. Scarlet................... 10

Meteor. Scarlet............................. 10

Mont Blanc. Pure white; early.............. 10

$\begin{array}{lll}\text { Mrs. Alex. Wallace. Lavender.................... } & 10 \\ \text { Mrs. F. J. Delansky. Daybreak-pink........... } & 10\end{array}$

Mrs. Sims. Salmon-pink. .................. 10

$1 / 41 b$

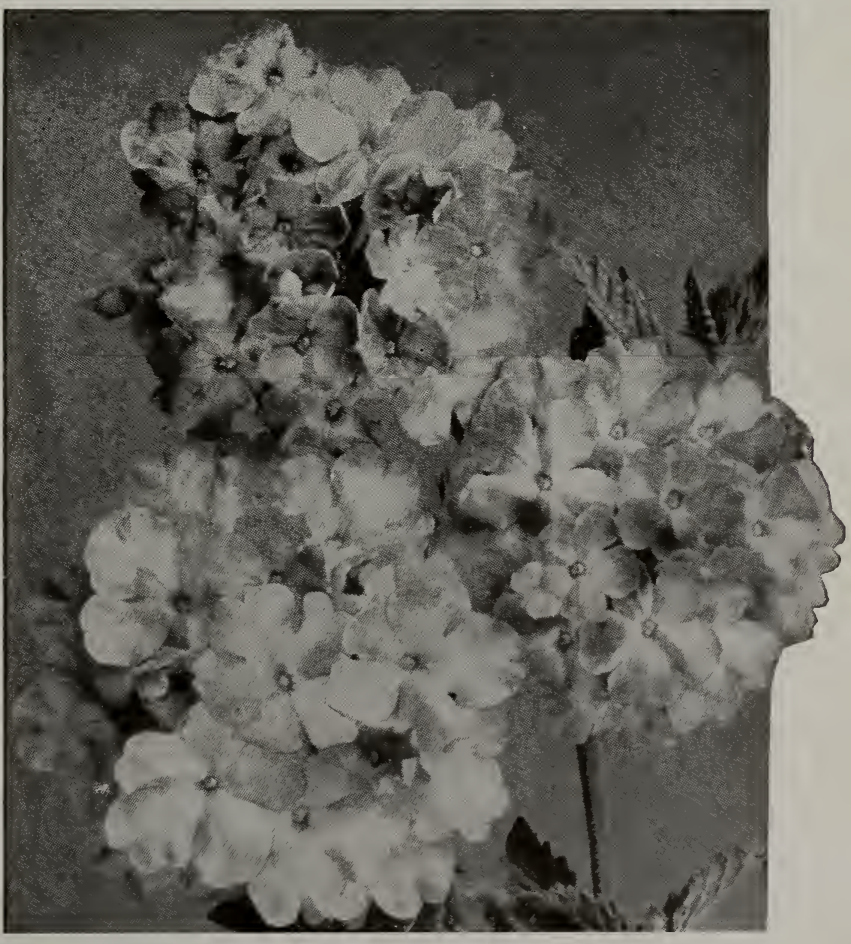

MAMMOTH VERBENA

\section{SWEET PEA MIXTURES}

These mixtures are prepared with special reference to blending colors in proper proportions. 1/4b. Lb. Barnard's Royal Mixture.................\$0 25 \$0 85 Spencers, Mixed ............................. 40 15c.. 150 Extra-Choice Mixtures..................... $20 \quad 70$ Mixed Light Colors...................... $20 \quad 70$

The prices quoted in this list do not include postage, except on seeds in packets, ounces and $1 / 4 \mathrm{lbs}$. When ordering larger quantities, bulbs, supolies, etc., add for postage at the parcel-post rates according to the zone in which you live. 


\section{A SELECTION OF VEGETABLE SEEDS}

Desirable sorts, of special interest to florists, for sowing under glass and otherwise. For varieties not mentioned here, see our General Catalogue, 100 pages. It will be mailed free on application

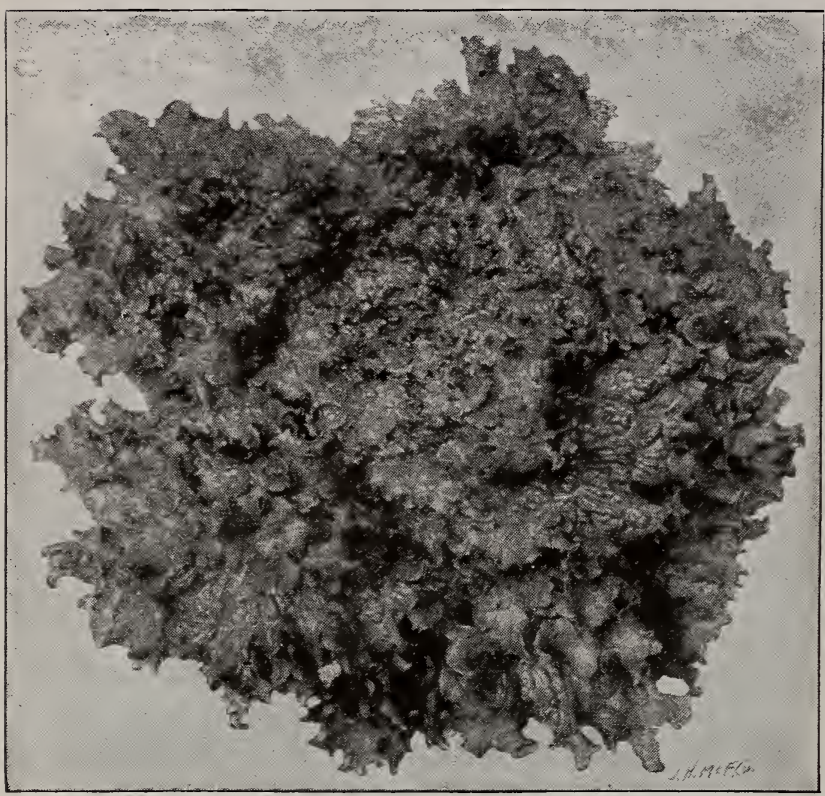

GRAND RAPIDS LETTUCE

\section{CABBAGE}

Early Jersey Wakefield. The best of the pointed-head varieties. Pkt. 5 cts., oz. 20 cts., $1 / 4 \mathrm{lb} .50 \mathrm{cts}$.

Copenhagen Market. Splendid, new, early variety; solid, ballshaped heads; ripens very uniformly. Pkt. 10 cts., oz. 25 cts., $1 / 4 \mathrm{lb} .90 \mathrm{cts}$.

Large Late Flat Dutch. Standard late variety. Pkt. 5 cts., oz. 20 cts., $1 / 4 \mathrm{lb} .50 \mathrm{cts}$.

\section{CAULIFLOWER}

Extra-Early Dwarf Erfurt. Special strain for growing under glass. Pkt. 15 cts., $1 / 4 \mathrm{oz} .60$ cts., oz. $\$ 1.75$.

Barnard's Snowball. The best dry weather or main-crop Cauliflower. Pkt. 15 cts., $1 / 4$ oz. 60 cts., oz. $\$ 1.75$.

\section{CELERY}

White Plume. The most popular of the early varieties. Pkt. 5 cts. oz. 15 cts., $1 / 4$ lb. 50 cts.

Winter Queen. Good winter variety. Pkt. 5 cts., oz. 15 cts., $1 / 4$ lb. 40 cts.

\section{CUCUMBER}

Telegraph. (Forcing.) Special strain for growing under glass. Pkt. 15 cts.

Davis Perfect. Fine white spine variety for either indoor or outdoor culture. Pkt. 5 cts., oz. $10 \mathrm{cts}$, $1 / 4 \mathrm{lb} .25 \mathrm{cts}$.

\section{EGGPLANT}

New York Improved. The best of the large purple varieties. Pkt. 5 cts., oz. $30 \mathrm{cts}$

\section{KOHLRABI}

Early White Short-leaved. Smooth, fine-grained variety; excellent for forcing. Pkt. 5 cts., oz. 20 cts., $1 / 4 \mathrm{lb} .65 \mathrm{cts}$.

\section{LETTUCE}

Grand Rapids. Growers of Lettuce under glass will find our special forcing strain of this variety to be of exceptional merit for greenhouse culture. Pkt. 5 cts., oz. 10 cts., 1/4lb. 30 cts., lb. $\$ 1$.

Big Boston. The favorite Head Lettuce for transplanting outdoors. Pkt. 5 cts., oz. 10 cts., $1 / 4$ lb. 30 cts., lb. $\$ 1$.

\section{PARSLEY}

Champion Moss Curled. We have a good strain of this variety for growing under glass. Pkt. 5 cts., oz. $10 \mathrm{cts.}, 1 / 4 \mathrm{lb}$. 30 cts., lb. $\$ 1$.

\section{PEPPER}

Chinese Giant. One of the best of the large, sweet sorts. Pkt. 5 cts., oz. 30 cts., $1 / 4 \mathrm{lb}$. $\$ 1$.

Celestial. Grown largely for ornamental purposes. Pkt. 5 cts., oz. 20 cts., $1 / 4 \mathrm{lb} .70$ cts.

\section{RADISH}

Barnard's Special Scarlet Globe Forcing. Color, uniformity of size, earliness and small growth of top are some of the qualities that have won the patronage of some of the largest Radish-growers around Chicago. Ask for "Special Forcing Strain." Pkt. 5 cts., oz. 10 cts., 1/4lb. 20 cts., lb. 70 cts.

Scarlet Turnip, White-Tip Forcing. Special strain of this popular white-tipped Radish for forcing under glass. Pkt. 5 cts., oz. 10 cts., $1 / 4 \mathrm{lb} .20$ cts., lb. $65 \mathrm{cts}$.

\section{TOMATO}

John Baer. New, early, bright red Tomato; the largest of the extra-early sorts; ripens evenly and close to the stem. A splendid variety either for the early market or the home garden. Pkt. 10 cts., oz. 40 cts.

Comet. A scarlet Tomato of medium size, especially suitable for growing under glass. Pkt. $10 \mathrm{cts}$, oz. $40 \mathrm{cts}$.

June Pink. The earliest of the pink Tomatoes. Good variety to start early for plants. Can be grown under glass; also excellent for the home garden. Pkt. 5 cts., oz. 20 cts., $1 / 4 \mathrm{lb}$. $70 \mathrm{c}$.

Beauty. Round, smooth, purplish pink. A favorite with market-gardeners as a main-crop sort. Pkt. 5 cts., oz. 15 cts., $1 / 4 \mathrm{lb} .50 \mathrm{cts}$.

Earliana. The earliest scarlet. Pkt. 5 cts., oz. 20 cts., 1/4lb. 65 cts. Golden Queen. Large; yellow. Pkt. 5 cts., oz. 20 cts., 1/4lb. 50 cts. Yellow Plum. Pear-shaped. Pkt. 5 cts., oz. 20 cts., 1/4lb. 50 cts.

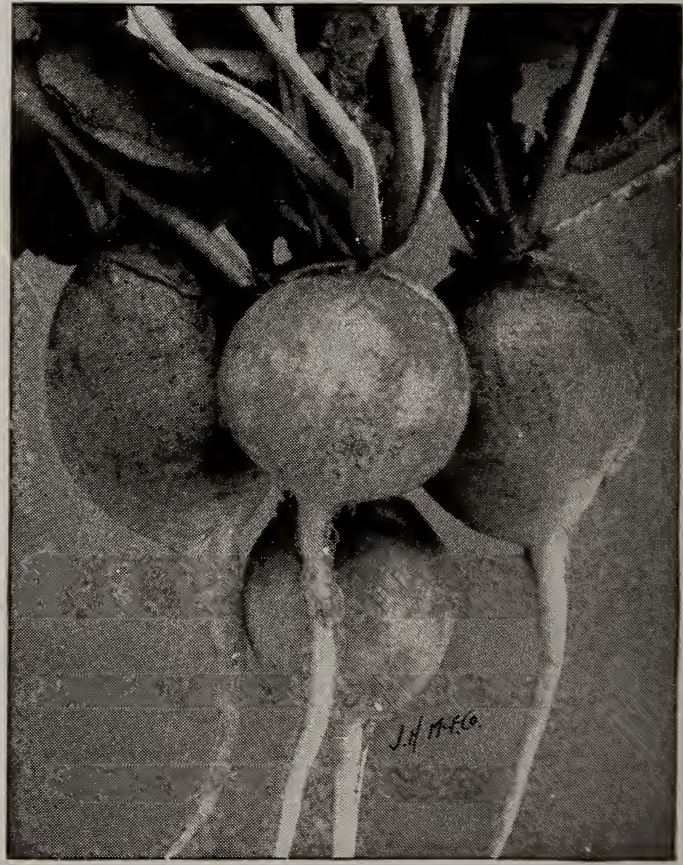

BARNARD'S SPECIAL SCARLET GLOBE RADISHE 


\section{BULBS FOR SPRING PLANTING}

For the present season we are better prepared than ever to supply first-class, well-cured bulbs, the best the market affords. We invite the custom of those who appreciate our endeavors to supply best stock. We are not responsible for any damage caused by delay, heat or cold while in transit, by freight.

We supply 6 bulbs at dozen rate, 25 bulbs at 100 rate, 250 bulbs at 1,000 rate

\section{CALADIUM ESCULENTUM}

Sound, well-cured stock

Grown especially for our trade; they are in prime condition, solid and firm, and have good live center shoots.

5 to 7 inches in circumference ....\$0 35 . $\$ 1 \quad 75$ 7 to 9 inches in circumference.... $50 \quad 325$ 9 to 11 inches in circumference.... $85 \quad 600$ 11 to 12 inches in circumference.... 1351000 12 inches and up in circumference... 2001400

\section{CANNAS, NAMED SORTS}

Dormant tubers, with two or three eyes each.

This list has been carefully revised and the best of the newer Cannas included. Cnless stated to the contrary, foliage is green. Early orders for this stock are advisable.

\section{Large Orchid-Flowering}

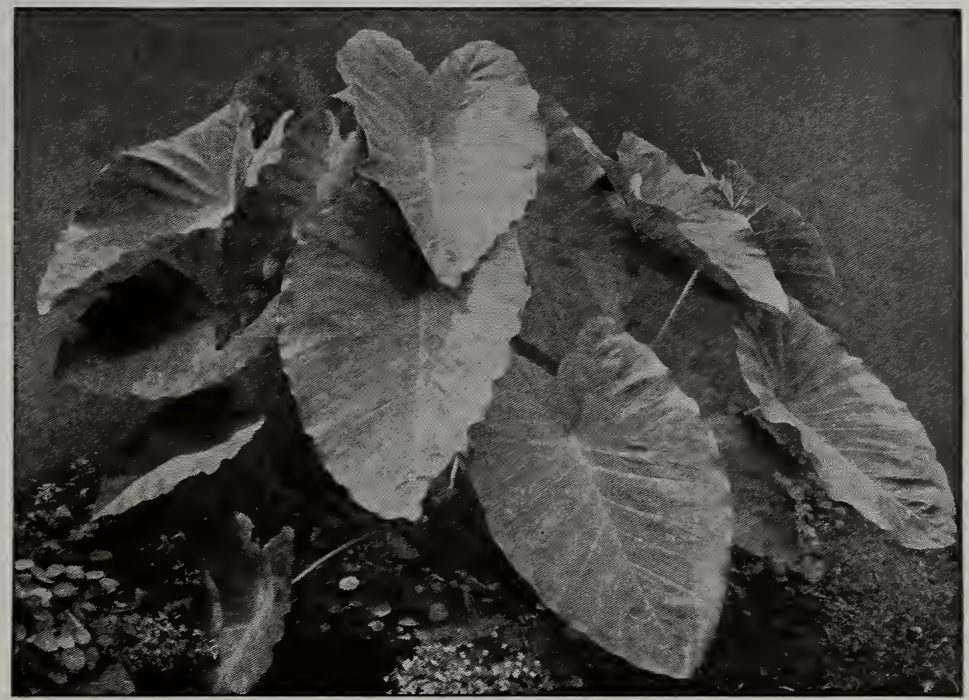

Austria. $6 \mathrm{ft}$. Canary-yellow, spotted red... Burbank. 4 to $5 \mathrm{ft}$. Clear yellow. .

Louisiana. $6 \mathrm{ft}$. Large flowers of vivid scarlet. Mrs. Kate Gray. $6 \mathrm{ft}$. Scarlet and yellow. .

Pennsylvania. 5 to $6 \mathrm{ft}$. Brilliant scarlet.

Wyoming. $6 \mathrm{ft}$. Rich orange flowers; bronze foliage

\begin{tabular}{rrrr} 
& & \multicolumn{2}{c}{ Doz. } \\
.$\$ 0$ & 50 & $\$ 3$ & 00 \\
. & 40 & 2 & 50 \\
. & 40 & 2 & 50 \\
. & 40 & 2 & 50 \\
. & 40 & 2 & 50 \\
. & 50 & 3 & 00
\end{tabular}

\section{Yellow-Flowering}

Florence Vaughan. $41 / 2 \mathrm{ft}$. Yellow, spotted crimson. Gladiator. $4 \mathrm{ft}$. Yellow, spotted red.

Richard Wallace. $5 \mathrm{ft}$. Canary-yellow; large flowers.
$40 \quad 250$

50300

$40 \quad 250$

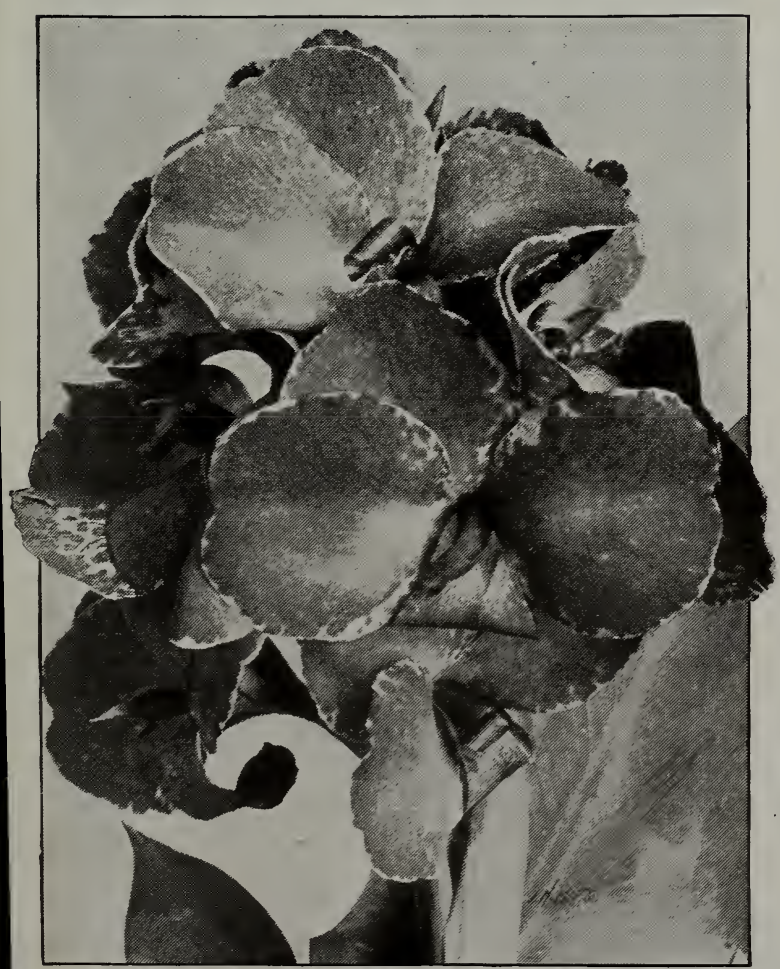

ORCHID-FLOWERING CANNA
CALADIUM ESCULENTUM

\section{$\underline{\text { Red-Flowering }}$}

\begin{tabular}{|c|c|c|}
\hline Alphonse Bouvier. $6 \mathrm{ft}$. Crimson. & $\begin{array}{l}\text { Doz. } \\
\$ 0 \\
50\end{array}$ & $\$ 300$ \\
\hline Chas. Henderson. $31 / 2 \mathrm{ft}$. Bright crimson. & 50 & 300 \\
\hline Feuermeer. $4 \mathrm{ft}$. Fiery scarlet; free-flowering....... & j0 & 300 \\
\hline 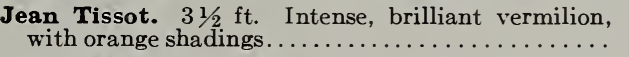 & 50 & 300 \\
\hline J. D. Eisele. $31 / 2 \mathrm{ft}$. Bright, vivid scarlet......... & 40 & 250 \\
\hline $\begin{array}{l}\text { irebird. } 41 / 2 \mathrm{ft} \text {. Clear glisten } \\
\text { any streaks or blotches. . . . . . . }\end{array}$ & & \\
\hline
\end{tabular}

\section{White- and Pink-Flowering}

Mue. Berat. $41 / 2 \mathrm{ft}$. Carmine-pink.................. $40 \quad 250$

Mont Blanc, Improved. $3 \mathrm{ft}$. Pure white.......... 65 . 500

Mrs. Alfred F. Conard. $4 \mathrm{ft}$. Salmon-pink....... $65 \quad 450$

Venus. $4 \mathrm{ft}$. Rosy pink, with a mottled border of canary-yellow......................... $45 \quad 275$

\section{Variegated-Flowering}

Mad. Crozy. $2 \frac{1}{2} \mathrm{ft}$. Scarlet, gilt edge............ $40 \quad 250$

Niagara. $3 \mathrm{ft}$. Rich, deep crimson, wide, irregular border of deep golden yellow....

Queen Charlotte. $3 \mathrm{ft}$. Scarlet, gilt edge.

Souv. de A. Crozy. $31 / 2 \mathrm{ft}$. Crimson, with gold band.

\section{Bronze Foliage}

Egandale. $4 \mathrm{ft}$. Currant-red flowers............ $60 \quad 3 \quad 50$

Robusta. $7 \mathrm{ft}$. Red flowers................ $50 \quad 300$

King Humbert. $4 \mathrm{ft}$. Orange-scarlet, with red markings...

Rosea gigantea. $5 \mathrm{ft}$. Large rose-pink flowers. . . .

Rubin. $41 \frac{1}{2} \mathrm{ft}$. Rich crimson...

Wm. Saunders. $4 \mathrm{ft}$. Bright scarlet; flowers often $\tilde{5}$ inches across, produced in great clusters on branching stems...........

\section{Cannas in Mixture}

Red Shades. Mixed.................... $40 \quad 275$

Yellow Shades. Mixed.

White and Pink. Mixed...

Variegated-flowering. Mixed

Orchid-flowering. Mixed.

Bronze-foliaged Varieties

All Sorts, Mixed
$60 \quad 4 \quad 00$

$60 \quad 475$

55350

$75 \quad 500$ 


\section{Selected and Best Dahlias for Florists}

We wish to call special attention to the free-flowering and early-blooming qualities of these splendid new Dahlias, which make them desirable sorts for florists.

\section{All Dahlias offered on this page are field-grown roots}

AUGUSTA NONIN. Decorative. One of the most brilliant Dahlias in existence, the color being a rich, fiery scarlet. Blooms are of the largest size, full in the center and of the best exhibition type. The plants are of free and upright growth, and produce quantities of large flowers, on long, stiff stems, with ease. Doz., \$1.75; 100, \$13.

DELICE. Decorative. The finest Dahlia introduced within the last six years. At no time has any Dahlia created such a worldwide popdlarity as has this sterling sort, within the last year. In color it is a beautiful shade of rosy pink, suffused with lavender-pink. The flowers are medium in size, of the most perfect decorative form, and are produced in the utmost profusion on long stems and held up well above the foliage. This variety should appeal to every lover of flowers on account of its delicate color. Doz., $\$ 2 ; 100, \$ 15$.

GOLIATH. Cactus. A monster, yet a sterling good sort, as from early August until frost ended their career, our plants of this continued to produce flowers of surprising size, with perfect centers and on long, strong stems, making them ideal for cutting. The color is yellow, suffused and tinted with reddish salmon. Doz., $\$ 1.40 ; 100, \$ 10$.

KAISERIN AUGUSTA VICTORIA. Decorative. Another sterling decorative sort that has created quite a sensation. The color is a clear ivory-white, without a shade or blemish; the flowers are large, of perfect form, with immense, broad, flat petals gracefully curved. Doz., $\$ 1.60 ; 100, \$ 11$.
MASTER CARL. The King of the Cactus Dahlias. In this grand variety we have all that can be wanted; its color is a pleasing shade of bright amber, which, combined with its other good qualities, make it unsurpassable for exhibition or garden purposes. Doz., $\$ 1.75 ; 100, \$ 12$.

MONT BLANC. Decorative. One of the finest varieties it has been our good fortune to list. The color of this sterling novelty is a clear ivory-white, without the usual green center most always noted in white Dahlias. This variety can be used for either garden, cutting or exhibition. Doz., $\$ 2 ; 100, \$ 15$.

PIUS $\mathbf{x}$. In this we believe we are offering the ideal white Cactus Dahlia, which has been a desideratum for a long time. In habit of growth it is robust and in color a pure snow-white, with flowers from 5 to 6 inches in diameter. Doz., $\$ 1.75 ; 100, \$ 12$.

PAUL KRUGER. Pæony-flowered. A very pleasing blending of purplish maroon and white. The plant is a very early and prolific bloomer, producing its flowers on extremely long stems. Doz., $\$ 1.75 ; 100, \$ 12$.

QUEEN WILHELMINA. Pæony-flowered. Immense, fluffy flowers of pure white, with yellow center, the petals being very peculiarly twisted and incurved; the flowers are produced on extra-long stems, making them invaluable for cutting or garden decoration. Doz., $\$ 1.75 ; 100, \$ 13$.

VIRGINIA MAULE. Decorative. A gem of the most beautiful coloring, being a delicate shade of shell-nink. Plants robust and flower abundantly throughout the season. Doz., \$1.75; 100, \$12. Mrs. A. H. Goodwin. New Decorative Dahlia. Our own introduction. Pure white with fine lavender center. Petals

\section{Standard Dahlias}

Arabella. Show. Primrose, edged with rose, shaded Doz. 100 lavender... $\$ 120 \$ 800$

A. D. Livoni. Show. Soft pink; quilled petals; fine for cutting......................... $120 \quad 800$ Catherine Duer. Decorative. Crimson-scarlet, darker at center...................... $120 \quad 800$

Countess of Lonsdale. Cactus. Salmon-pink; perfect in form..................... $120 \quad 800$



DAHLIA, JACK ROSE
Clifford W. Bruton. Decorative. Canary-yellow; Doz. 100 one of the best ...................... \$1 20 $\$ 800$

Earl of Pembroke. Cactus. Plum-color........ $120 \quad 800$

Flora. Cactus. Pure white; broad petals.......... 1351000

Floradora. Cactus. Dark, velvety crimson; early and profuse bloomer............... 125900

Frank Smith. Show. Maruon, almost black; sometimes tipped white $\ldots \ldots \ldots \ldots \ldots \ldots \ldots \ldots \ldots$ Gaillard. Cactus. Crimson.............. $120 \quad 800$ Gaillardia. Single. Yellow and red......... 1208800 Grand Duke Alexis. Cactus. White and pink; unique in form. Harbor Light. Cactus. Orange-red.......... $120 \quad 800$ Henry Patrick. Cactus. White.......... 120800 Jack Rose. Decorative. Crimson........... $120 \quad 800$ Lyndhurst. Decorative. Bright red......... 120800 Matchless. Cactus. Crimson and maroon..... 120800 Miss May Lomas. Show. White, suffused pink. ... Mrs. Roosevelt. Giant. Delicate silvery rose; a magnificent flower.

Penelope. Show. White, striped lavender...... 1208800

Perle d'Or. Decorative. Glistening white ..... 1208800

Progress. Fancy. Soft, rosy lake.......... 1259900

Queen Victoria. Show. Deep yellow ........ 1208800

Scarlet Ball. Show ................ 120800

Snowclad. Pompon. Pure white........... 120800

Storm King. Show. White............. 1351000

Sylvia. Decorative. Soft pink........... 1208800

Twentieth Century. Single. Crimson........ $120 \quad 800$

Wm. Agnew. Decorative. Scarlet......... 120800

White Century. Single. Snow-white........ 1208800

\section{Dahlias Under Color}

For many purposes these unnamed sorts prove fully as satisfactory as more expensive varieties.

White

Red

Pink

Yellow

$\$ 1$ per doz., \$6 per 100

Write us now to put your name on our mailing list, so you will be sure to receive a copy of our Wholesale List of Bulbs and Seeds for Fall Planting-ready about the first of September 


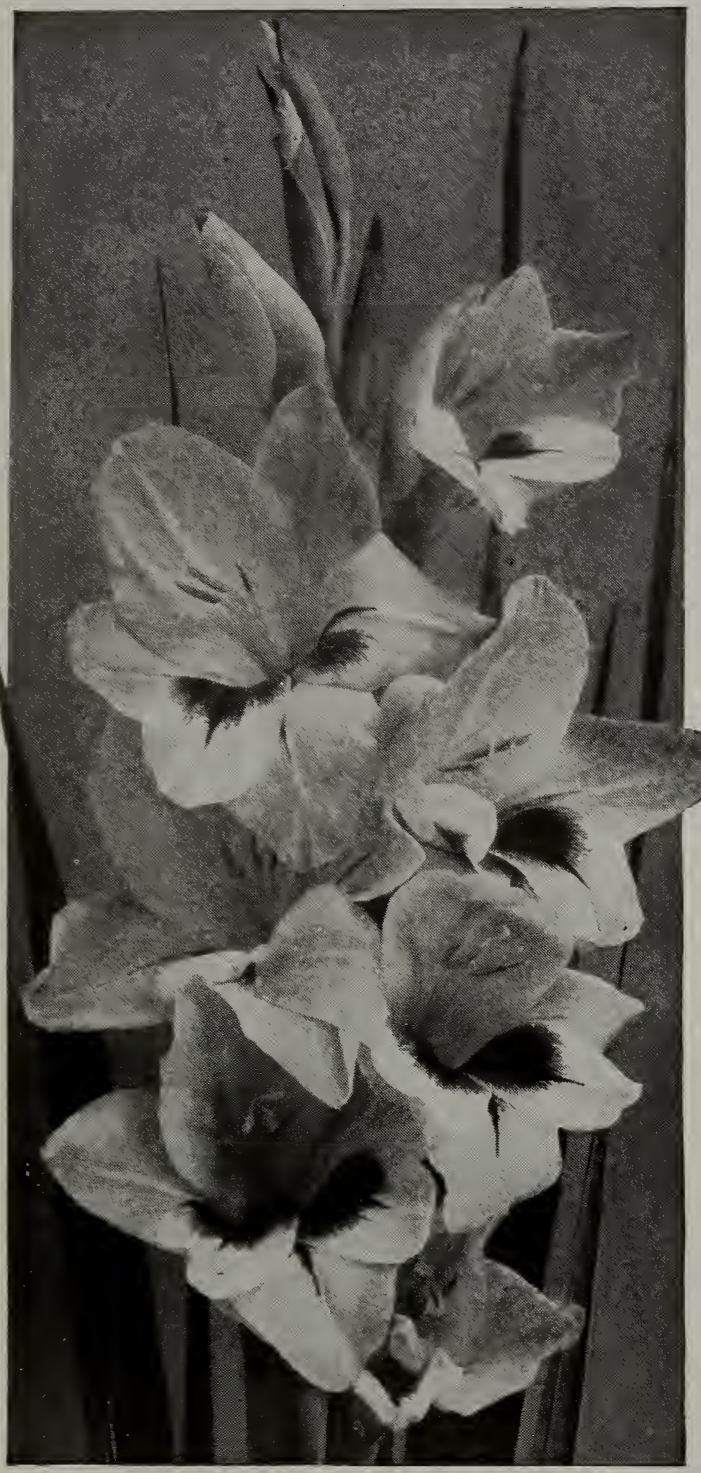

LEMOINE'S GLADIOLUS

\section{HARDY JAPAN LILIES \\ READY TO DELIVER NOW}

The Japan Lilies offered below, if planted out-of-doors during the early spring months, should bloom in July and August. Many florists find them very useful for decorative purposes. They do well planted among shrubbery, as they are benefited by the shade afforded by surrounding plants.

25 bulbs at 100 rate, 250 at 1,000 rate First-class Sto ck Doz. 100

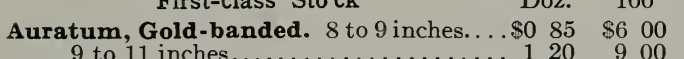

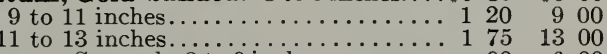
ubrum. Spotted. 8 to 9 inches............ 90 9 900 9 to 11 inches................... $140 \quad 1000$ 11 to 13 inches....................... $21200 \quad 1400$

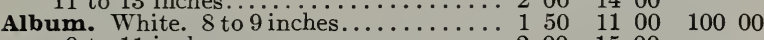
9 to 11 inches..................... $200 \quad 1500$ Magnificum. Rich blood-crimson spotted. 8 to 9 inches..................... $90 \quad 600$ 9 to 11 inches................... $140 \quad 1000$

1,000 $\$ 5 \overline{5} 00$ 8000 5500 5500 9000

\section{Tiger Lilies}

Tigrinum (Single Tiger Lily)...............\$0 40

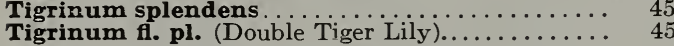

\section{Japanese Fern Balls, Dormant}

Pretty and attractive novelty. Finds ready sale when in leaf.

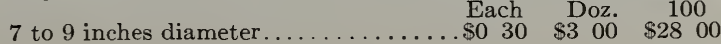

\section{GLADIOLI}

When larger quantities than here quoted are desired, write for special prices.

\section{Named Sorts}

AMERICA. Flesh-pink. Large size

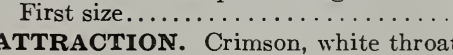

AUGUSTA. White; very fine

BARON HULOT. Rich indigo-blue.

BRENCHLEYENSIS. Vermilion-scarlet

CERES. Pure white, spotted with rose.

CHICAGO WHITE. White, with faint lavender

streaks; fine bedder............... $45 \quad 275$

DAZZLER. Salmon-scarlet.................. $250 \quad 2000$

INDEPENDENCE. Salmon-rose, velvet blotch. .

IRENE. Pink, flaked crimson...............

ISAAC BUCHANAN. Yellow; very fine.

Doz. $\quad 100 \quad 1,000$ $\begin{array}{lllllll}\$ 0 & 30 & \$ 1 & 45 & \$ 12 & 00\end{array}$ $\begin{array}{lllll}25 & 1 & 25 & 10 & 00\end{array}$

$\begin{array}{lllll}40 & 2 & 50 & 20 & 00\end{array}$

$\begin{array}{llllll}25 & 1 & 45 & 12 & 00\end{array}$

$\begin{array}{llllll}25 & 1 & 85 & 16 & 00\end{array}$

$\begin{array}{llllll}25 & 1 & 45 & 12 & 00\end{array}$

$\begin{array}{lllll}35 & 2 & 00 & 18 & 00\end{array}$

$\begin{array}{rrr}50 & 20 & 00 \\ 65 & 4 & 00\end{array}$

145

500

$90 \quad 5 \quad 50$

KUNDERDI GLORY. Delicate cream-pink with

crimson stripe in the center of each lower petal; flowers are ruffled and fluted like orchids...

MADAME MONNERET. Delicate rose.

MAY. White, striped crimson... .

MRS. BEECHER. Rosy crimson.............

MRS. FRANCIS KING. Light scarlet. Large size.

NIAGARA. Creamy blush............... 100

PRINCEPS. Crimson, white blotches.......... 40

ROSY SPRAY. White and rose . . . . . . . . . 60

SCRIBE. White, striped carmine...

SHAKESPEARE. White, flaked rose

\section{Gladioli in Mixtures}

$\begin{array}{lllllll} & & & & & & \end{array}$

triped and Variegated. Very choice.......... $4 \begin{array}{llllll}45 & 2 & 50 & 22 & 00\end{array}$

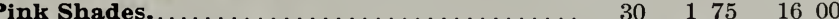

Childsii. Giant-flowered.................... $30 \quad 2 \quad \begin{array}{lllll} & 00 & 18 & 00\end{array}$

Superfine Mixed....................... $30 \quad 125 \quad 1000$

Good Mixed......................... $20 \quad 100 \quad 800$

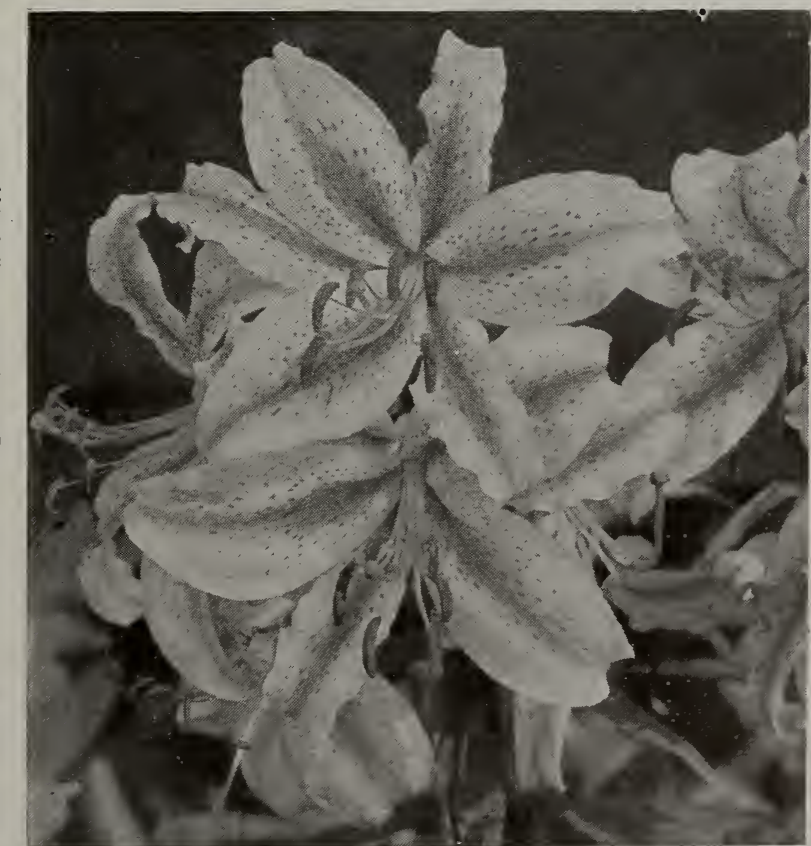

LILIUM AURATOM 


\section{Miscellaneous Bulbs and Roots}

\begin{abstract}
AMARYLLIS

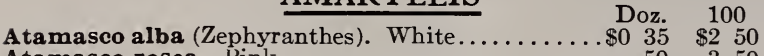

Atamasco rosea. Pink....................... 50 50 350

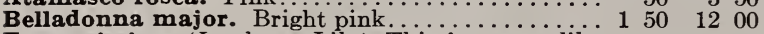

Formosissima (Jacobæan Lily). This forces readily.

Rich, velvety crimson flowers.

Johnsoni. Large flower; crimson, striped with white.

$80 \quad 550$

2251800
\end{abstract}

\section{TUBEROUS-ROOTED BEGONIAS}

Our Tuberous-rooted Begonia bulbs are large in size, well ripened and first-class in every respect. In our mixtures are included the best and most improved varieties only.

Single \#arieties in separate colors: White, Yellow, Doz. 100 Scarlet, Pink, Crimson, Orange................ \$0 40 $\$ 2 \quad 50$

Single Varieties, Mixed .................... $35 \quad 225$

Single Fringed Varieties, Mixed................ $45 \quad \begin{aligned} & 35 \\ & 3\end{aligned}$

Double Varieties, in separate colors: White,

Yellow, Scarlet, Pink..................... $60 \quad 400$

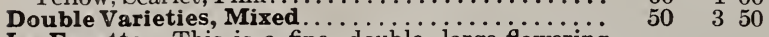

La Fayette. This is a fine, double, large-flowering

variety; fiery red in color.................. $175 \quad 1200$

\section{CINNAMON VINE}

First size, 10 to 18 inches

Second size, 6 to 10 inches

Third size, 3 to 6 inches.

$\begin{array}{lll}60 & 4 & 00 \\ 50 & 2 & 75\end{array}$

$\begin{array}{lll}50 & 2 & 75 \\ 35 & 2 & 00\end{array}$

CALLA, SPOTTED LEAF

Richardia maculata. White flowers.

$50 \quad 350$
DIEL YTRA spectabilis (Bleeding Heart). A well- Doz. 100 known favorite plant for forcing purposes........ $\$ 1 \quad 10 \quad \$ 800$

\section{GLOXINIAS}

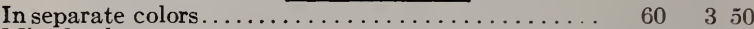

Mixed colors............................... $50 \quad \begin{array}{r}50 \\ 30\end{array}$

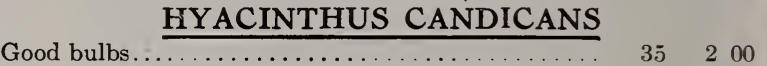

MADEIRA VINE

Good sound tubers.

Small size.

Medium size...

Select size....

\section{OXALIS, SUMMER-BLOOMING}

Pink, or White. In mixture

$100 \quad 1,000$

Deppei. Light crimson

Lasiandra. Purple.

$\begin{array}{llll}10 & 35 & \$ 2 & 50\end{array}$

Mixed. All sorts.

\section{TIGRIDIAS}

Mexican Shell Flower. Red, White, Yellow, or Doz. 100 Mixed ............................ $40 \quad 40 \quad \$ 250$

\section{TUBEROSES}

Quality exceptionally good this season.

Dwarf Pearl. Large bulbs.

Second-size bulbs. .

$\begin{array}{cc}100 & 1,000 \\ \$ 100 & \$ 900\end{array}$

$60 \quad 450$

\section{Hardy Herbaceous Peonies}

We offer this season a collection of especially selected Peonies, in strong divisions, with three to five eyes each.

\section{White and Light Varieties}

Alba plena. Pure white; double; fine- Each Doz. 100 scented........................ \$0 $20 \quad \$ 175 \quad \$ 1500$

Couronne d'Or. Large, snowy white blooms, with golden yellow stamens and delicate carmine markings; strong, upright grower. One of the choicest of the late-flowering varieties.

Duchesse de Nemours. A very fine, cupshaped bloom; sulphur-white, changing to pure white. Has a fine bud and is particularly beautiful when half open

Festiva maxima. Generally considered the finest early white Peony. Large, globular type; pure white, flecked with crimson; type, purrant; tall, vigorous grower

Marie Lemoine. Large, pure white bloom of perfect shape, slightly shading to chamois, narrow carnine edge; very late; magnificent; fragrant; medium height.

Mme. de Verneville. Beautifully formed flower; pure white, with very broad, sul phur-white guard petals; compact centers, delicate rosy white touched with carmine. Grand midseason variety

Queen Victoria. A strong-growing, pure white variety; with creamy white center one of the best early cut-flower varieties. .

$25 \quad 200 \quad 1600$

$\begin{array}{lllll}35 & 3 & 25 & 20 & 00\end{array}$

$35 \quad 3 \quad 60 \quad 2500$

$\begin{array}{lllll}30 & 2 & 25 & 16 & 00\end{array}$

$25 \quad 200 \quad 1500$

\section{Red Varieties}

Louis Van Houtte. Rich, dark carmine, Each Doz. 100

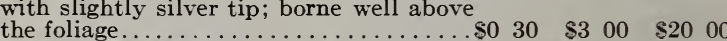
the foliage......................... full bloom

$35 \quad 360 \quad 2500$

Rubra triumphans. "The old favorite crimson, which has stood the test of time $\begin{array}{llllll}25 & 2 & 00 & 15 & 00\end{array}$ Officinalis rubra. Deep red............ $20 \quad 20 \quad 200 \quad 1400$

\section{Pink and Rose Varieties}

Edulis superba. Large, loose bloom of per- Each Doz. fect shape; bright mauve-pink, with lilac edge; fragrant. A free and early bloomer... $\$ 0 \quad 35 \quad \begin{array}{lll}\$ & 60\end{array}$

Lady Leonora Bramwell. Beautiful silvery rose; of fine form; free-blooming, vigorous-growing variety. The beautiful orous-growing variety. The beautiful, soft-colored flowers make it very attractive for midseason flowering.................
Madame Chaumy. Large; bright lilac-rose blossoms, with silvery reflex. One of the most desirable late-blooming varieties..... Meissonier. Large blooms on long stems; beautiful pink; fragrant; late; stronggrowing variety....................

\section{Unnamed Peonies Under Color}

Red, Pink, White

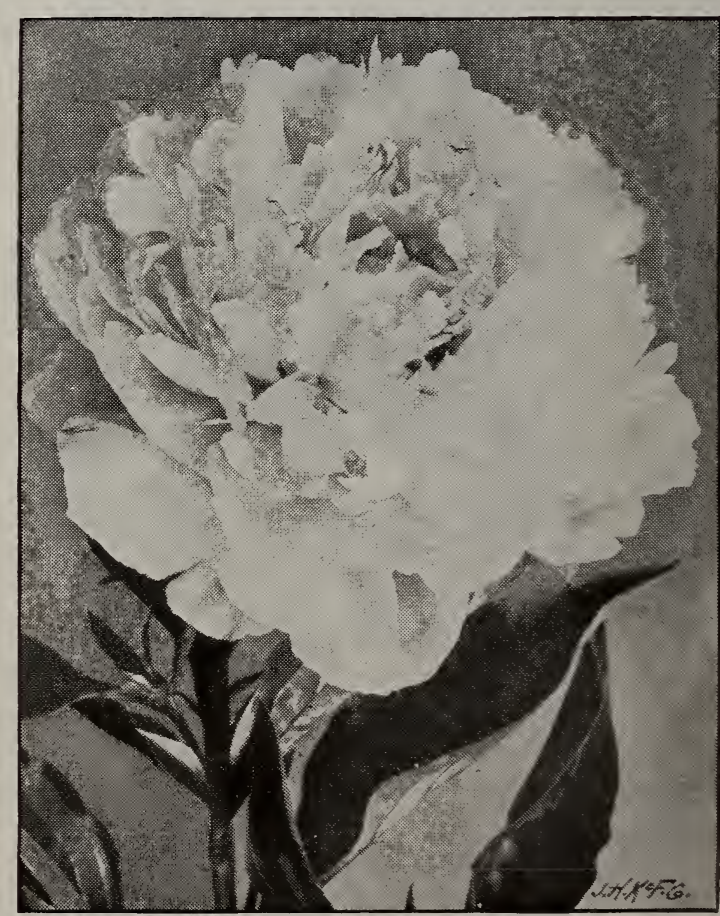




\section{Hardy Roses and Herbaceous Perennials}

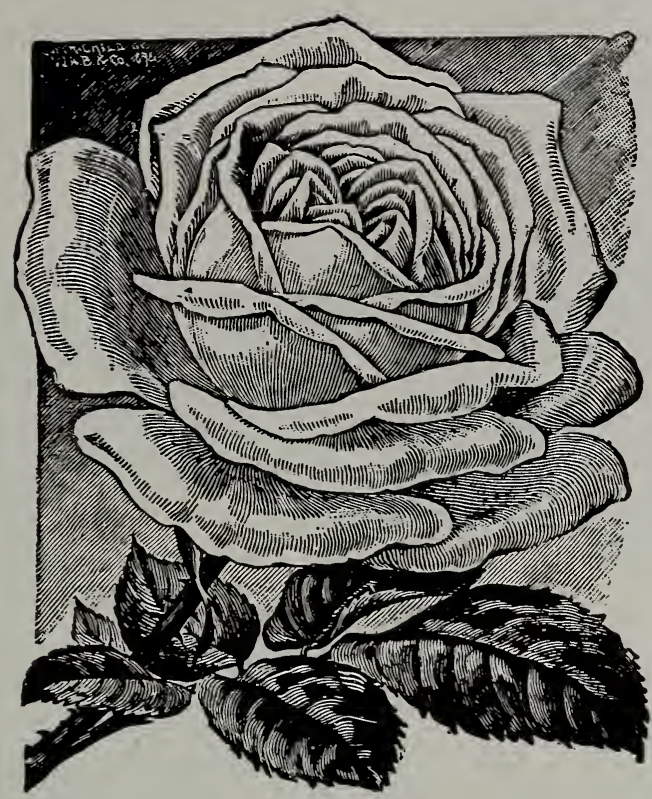

\section{HYBRID PERPETUAL ROSES}

White, pink, crimson and yellow.

Selected Dormant Stock. Ready About April 1st. Fach 20c; doz., \$2.00; 100, $\$ 14.00$

The list below, while not extensive, includes the most popular varieties.

Alfred Colomb. Brilliant carmine crimson; fragrant. Anne de Diesbach. A beautiful shade of carmine. Ball of Snow. Pure white; best of its color. Baron de Bonstetten. Rich velvety maroon; full. Baroness Rothschild. Light pink; cupped form. Clio. Flesh color; handsome foliage; vigorous. Fisher Folmes. Rich crimson; moderately full. General Jacqueminot. Crimson; fragrant Mabel Morrison. White tinged with blush. Madam Gabriel Iuizet. Pink; cup-shaped. Madame Plantier. Pure white; fine for hedges. Madame victor verdier. Carmine crimson; large. Magna Charta. Light pink.

Mrs. John Iaing. Soft pink; fine form; large. Paul Neyron. Deep rose color; good forcer. Prince Camille De Rohan. Deep crimson. Ulrich Bruner. Bright cherry-red; fine form.

\section{EVERBLOOMING ROSES}

Each, 20c; doz., \$2.00; 100, $\$ 15.00$

These Everblooming Roses produce a wealth of bloom during the summer and fall months.

American Beauty. Deep rose; large; very fragrant. Captain Christy. Flesh color; medium full.

Frau Karl Druschi. White, large and beautiful.

Gruss an Teplitz. Dark velvety crimson.

Killarney. Pink: a well known rose.

Ia France. Silvery rose; fine form.

Madame Caroline Testout. Salmon pink; fine.

\section{PERSIAN YELLOW AND TREE}

\section{ROSES}

Brier. Persian yellow; each, 20c; doz., \$2.00; 100, $\$ 16.00$.

Tree. Well-shaped plants, budded. Assorted colors. Each, 75 cts.; doz., $\$ 8.00$.

Crimson Rambler. Each, 60c.; doz., $\$ 6.00$

\section{RUGOSA ROSES}

Two-year-old Plants

Alba and Rubra. Each, 20 cts.; doz., $\$ 2.00 ; 100, \$ 15.00$.

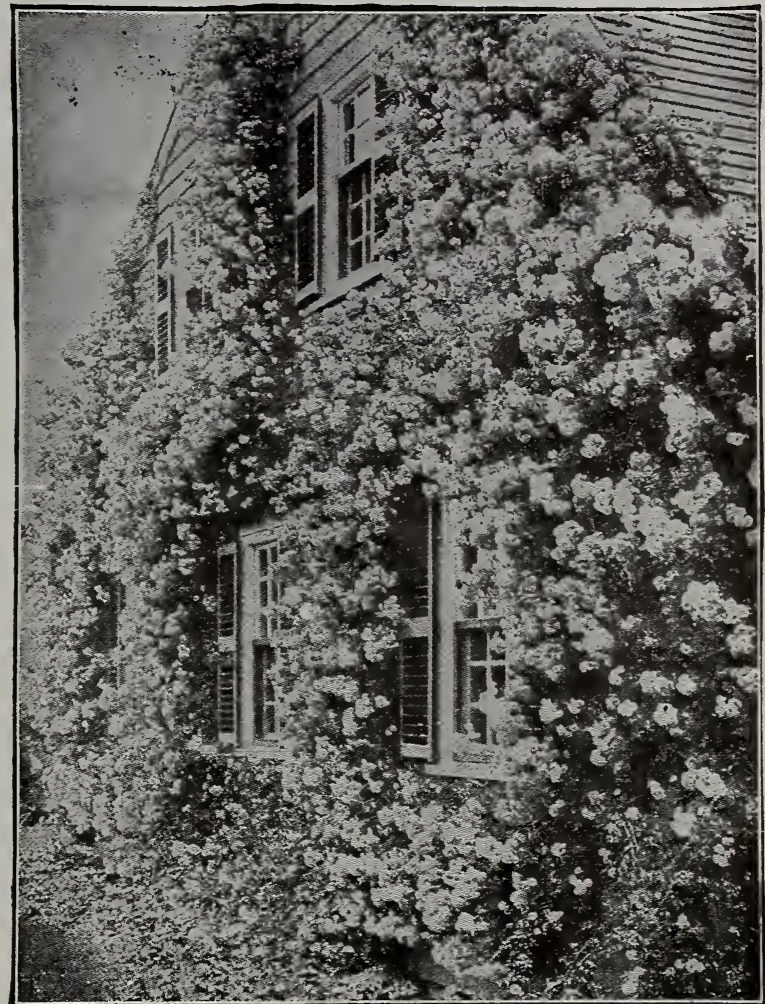

\section{RAMBLER AND CLIMBING ROSES}

Rambler. White, Pink, Crimson and Yellow. Each, Climbing. $\$ 2.50 ; 100, \$ 18.00$. white; Prairie Queen red changing to pink. Seven Sisters, shaded: Dorothy Perkins, shell pink; Tausendschoon, bright pink. Each, $30 \mathrm{c}$; doz., $\$ 2.50 ; 100, \$ 15.00$.

Crimson Baby Rambler. Each, 20c; doz., $\$ 2.00 ; 100$,

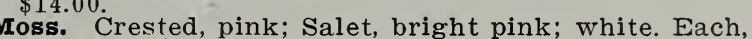
$20 \mathrm{c}$; doz., $\$ 2.00 ; 100, \$ 15.00$.

\section{HARDY OUTDOOR PLANTS}

FIELD-GROWN, DIVIDED ROOTS

Aquilegia (Colum

Achillea (Milfoil)

Asters, Hardy (Michælmas Daisy)

Bellis perennis (English Daisy)

Bleeding Heart (Dicentra spectabilis)

Campanula (Canterbury Bell)...

Chrysanthemum, Hardy (Shasta Daisy)

Maximum...

Coreopsis lanceolata.

Delphinium (Larkspur).

Dianthus barbatus (Sweet William)

Dianthus. Hardy Pinks................

Digitalis (Foxglove)

Eulalia (Striped Grass)

Funkia (Day Lily)

Gaillardia (Blanket Flower)

Helianthus (Sunflower).

Hibiscus, crimson eye.

Hollyhocks, double, assorted colors

Iberis, Perennial Candytuft.

Iris Kaempferi, or Japanese.

Japan, imported, very choice.

German, mixed colors.

Lily-of-the-Valley ..

Myosotis (Forget-me-not)

Phlox, hardy, assorted colors ...

Pinks, old-fashioned, hardy

Poppy orientale

Rudbeckia (Golden Glow).

Stokesia cyanea (Cornflower Aster)

Tritoma (Red-hot Poker Plant)

Vinca (Periwinkle). For cemeteries

Yucca filamentosa (Adam's Needle).

$10 \quad 100$ $0.70 \quad \$ 6.00$

$\begin{array}{ll}.70 & 6.00 \\ .85 & 7.00\end{array}$

$.50 \quad 4.00$

$1.00 \quad 8.00$

$\begin{array}{ll}1.00 & 8.00\end{array}$

$.70 \quad 6.00$

$70 \quad 6.00$

$1.00 \quad 8.00$

$\begin{array}{ll}.70 & 6.00\end{array}$

$70 \quad 6.60$

1.00 


\section{FLOWERING AND ORNAMENTAL HARDY SHRUBS}

Especial attention is called to the very superior quality of the nursery stock we supply. It is first-class in every respect, smooth, straight and handsome. Personal inspection of the stock would be appreciated. We call particular attention to our heavy plants. They are especially selected, of the best form, and are suitable for retail trade where immediate effects are desired. Prices on specimen plants furnished on application. Packing Extra on all plant orders.

packing charged for at cost. We deliver to express and railroad depots in Chicago, free of charge. For light shipments we advise express; larger quantities or heavy packages generally go safely by freight. Purchaser pays transportation charges. State if we shall ship by express or freight. Where no instructions are given we use our best judgment as to route, but will not be responsible for delays. Prices subject to market changes and stock being unsold when order is received.

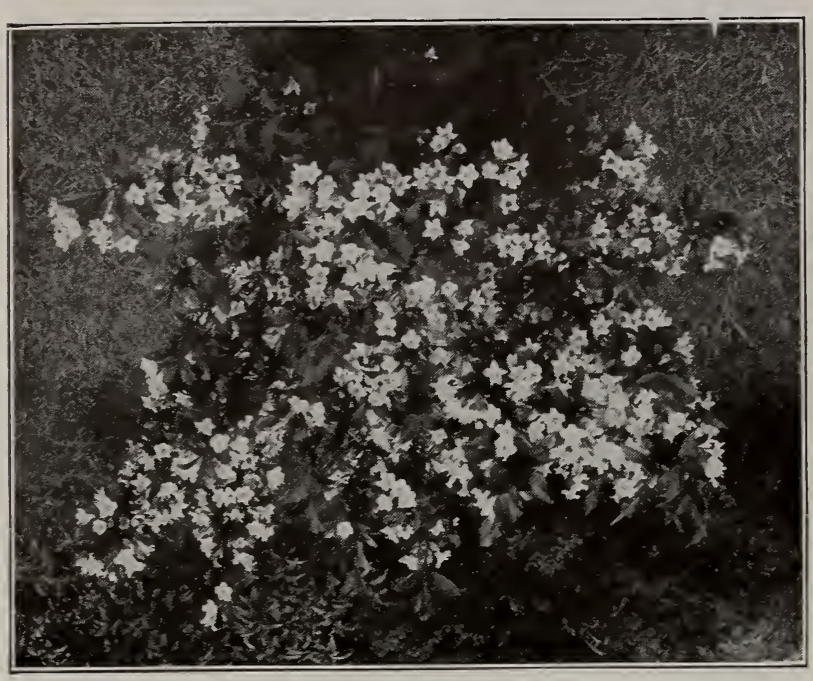

WEIGELIA CANDIDA

Our Nursery stock has been inspected for injurious insects and plant diseases and a certificate from the State Entomologist to that effect is supplied with each shipment.

\section{HARDY DECIDUOUS SHRUBS.}

AIMOND, Flowering Per 10 Per 100

White, 2 to 3 feet...................

AITHEA, (Rose of Sharon)

Double red, white and purple

3 to 4 feet...................... 2.50

Tree Shaped, 2 to $3 \mathrm{ft} \ldots \ldots \ldots \ldots \ldots .3 .50$

BARBERRY

Thunbergii, 15 to 18 inches........ 1.25 18 to 24 inches.........

Vulgaris, $\quad 2$ to 3 feet........... 1.25

Purpurea, $\quad 18$ to 24 inches.............

2 to 3 feet............

BURNING BUSH. See Euonymus.

BUSH HONEYSUCKIE. See Lonicera.

CAIYCANTHUS, (Spice-wood or Sweet-

scented Shrub) 2 feet........... 1.75

CORNUS

Sanguinea, Redbarked Dogwood.

2 to 3 feet

2 to 3 feet
3 to 4 feet

Sibirica. Crimson bark

CRAB, Bechtel's Double F'lowerin

3 to 4 feet, each $75 \mathrm{c}$.

CURRANT, Yellow-flowering (Ribes)

2 to 3 feet

\section{CYDONIA}

Japonica, (Japan Quince)

\section{DEUTZIA}

Iemoinei, 2 to 3 feet...........2.00

Pride of Rochester

$$
2 \text { to } 3 \text { feet................ } 1.50
$$

Gracilis, Nice Clumps.......... 2.00 DOGWOOD. See Cornus.

10.00

15.00

20.00

10.00

18.00

8.00

9.00

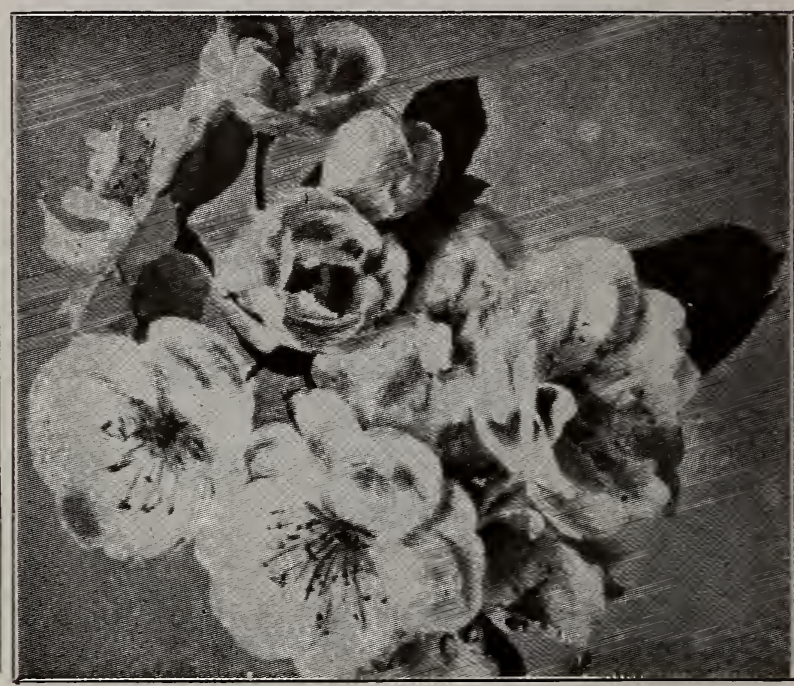

BECHTEL'S FLOWERING CRAB

FIDFR, (Sambucus)

Per 10 Per 100

Golden-leaved, 3 to 4 feet......... $\$ 2.50 \$ \$ 20.00$

Cut-leaved, 3 to 4 feet.........2.00 18.00

\section{EUONYMUS}

Alatus, (Strawberry or Spindle Tree)

3 to 4 feet, $\$ 1.00$ each.

European, (Burning Bush)

3 to 4 feet.................. 2.5

FORSYTHIA, (Golden Bell)

Fortunei 2 to 3 feet.......... $1.75 \quad 15.00$

3 to 4 feet.....................

Intermedia, 2 to 3 feet............ 2.00 18.00

Suspensa, heavy plants.........2.00

\section{HYDRANGFA}

Arborescens $\ldots \ldots \ldots \ldots \ldots \ldots \ldots, 3.50$

Paniculata Grandiflora

2 to 3 feet..................

15.00

Paniculata in tree form......... 5.00

\section{IIGUSTRUM}

Amurense (Amoor Privet)

18 to 24 inches............... 1.00

2 to 3 feet........................ 1.75

3 to 4 feet.............................

Ibota (Japan Privet)

18 to 24 inches............... $1.00 \quad 8.00$

2 to 3 feet...................... $1.50 \quad 12.00$

vulgaris (Common Privet)

2 to 3 feet....................... 2.00 12.00

3 to 4 feet........................ 2.50

IILAC

Vulgaris (Common Purple)

2 to 3 feet

$1.75 \quad 15.00$

3 to 4 feet

2.00

20.00

Vulgaris alba (Common White)

$$
2 \text { to } 3 \text { feet. }
$$

20.00

Persica (Purple Persian)

2 to 3 feet................................. 2.75

Marie Le Gray.................... 3.50

Charles X.............................. 30 


\section{HARDY SHRUBS, continued}

For full descriptions see Retail Catalogue. Free to Florists on request.

LONICERA (Bush Honeysuckle).

Tatarica

2 to 3 feet

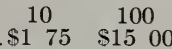

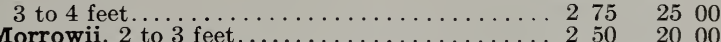

MAGNOLIA Soulangeana. 2 to 3 feet. \$2 each.

PHILADELPHUS (Mock Orange or Syringa).

grandiflorus. 2 to 3 feet....

$\begin{array}{ll} & 3 \text { to } 4 \text { feet.. } \\ \text { coronarius. } & 2 \text { to } 3 \text { feet.. } \\ 3 & \text { to } 4 \text { feet.. }\end{array}$

209

$\begin{array}{lll}2 & 75 \\ 2 & 00\end{array}$

$\begin{array}{ll}2 & 00 \\ 2 & 75\end{array}$

PRIVET. See Ligustrum.

PRUNUS Pissardii (Purple-leaved Plum)

2 to 3 feet...

250

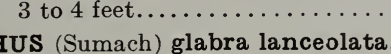

RHUS (Sumach) glabra lanceolata.

2 feet. laciniata (Fern Tree).

2 to 3 feet

copallina. 2 feet......................... $3_{3} 00$

SPIREA

Anthony Waterer.

18 to 24 inches. .

Billardii. 2 to 3 feet. White

2 to 3 feet. Pink.

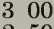

250

$\begin{array}{lll}1 & 50 \\ 1 & 50\end{array}$

$\begin{array}{ll}1 & 50 \\ 2 & 50\end{array}$

opulifolia (Ninebark). 3 to 4 feet.

opulifolia aurea (Golden-leaved).

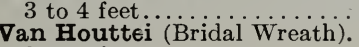

2 to 3 feet.

3 to 4 feet....

250

$\begin{array}{ll}1 & 50 \\ 2 & 00\end{array}$

$\begin{array}{ll}2 & 00 \\ 3 & 00\end{array}$

Heavy plants.

SNOWBALL. See Viburnum.

SYMPHORICARPOS.

racemosus (Snowberry).

18 to 24 inches..................

vulgaris (Coral Berry, Indian Currant)

2 to 3 feet......
Heavy plants

150

200

150

each 25 c... 2200

275

VIBURNUM

plicatum (Japan Snowball)

Opulus (High or Bush Cranberry)

pulus (High

2 to 3 feet
3 to 4 feet

Opulus sterile (Common Snowball).

2 to 3 feet.....

WEIGELA

candida. 2 to 3 feet

rosea. 2 to 3 feet.

Eva Rathke, 2 to 3 feet.

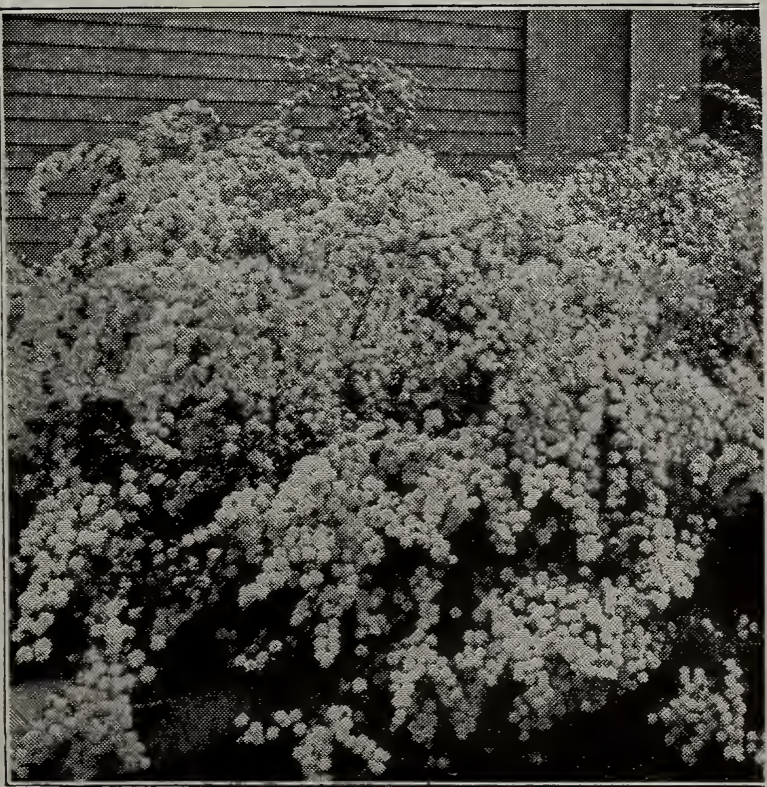

SFIREA VAN HOUTTEI (BRIDAL WREATH)

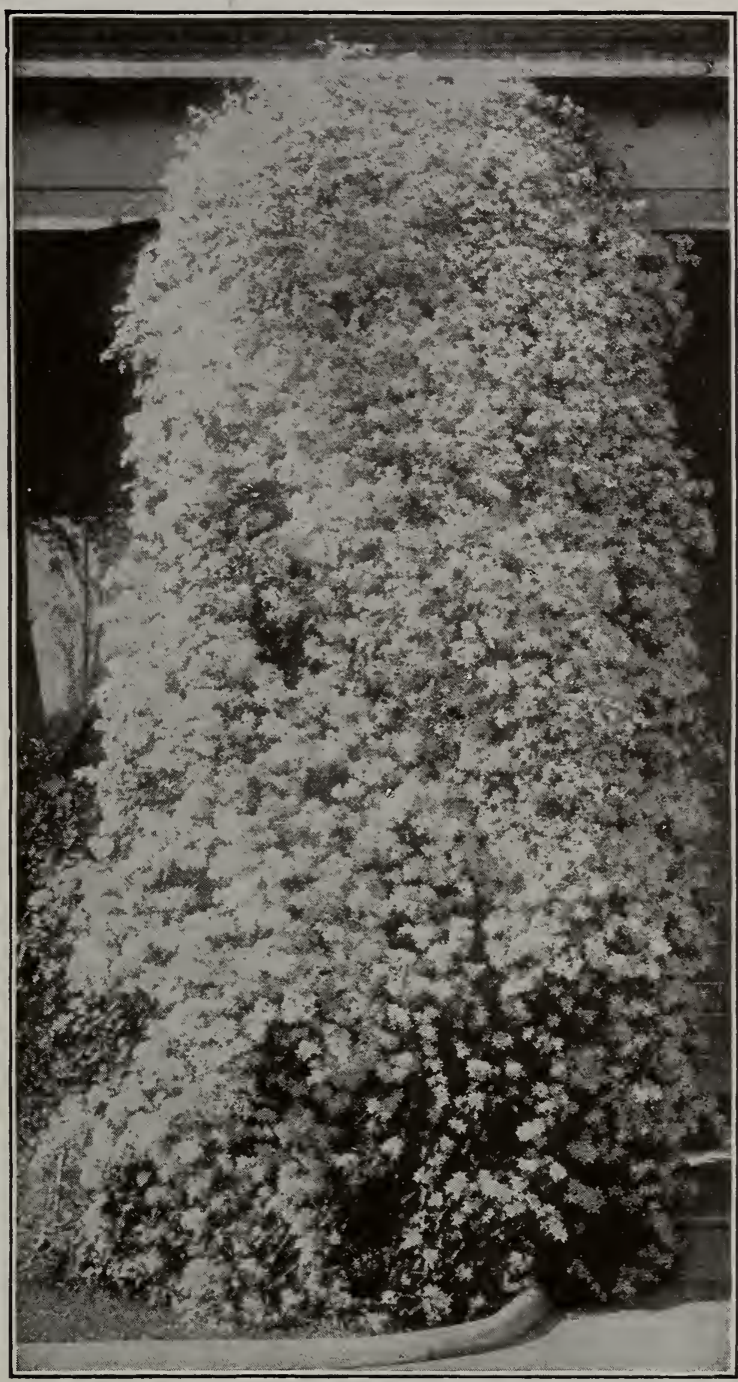

CLEMATIS PANICULATA

\section{VINES AND CLIMBERS}

\section{AMPELOPSIS}

Veitchii (Boston Ivy).

2 -year old....

3 -year old

Engelmannii

ARISTOLOCHIA Sipho (Dutchman's Pipe)

3-year plants.

$\begin{array}{llll}10 & 50 & \$ 12 & 00\end{array}$

$\begin{array}{llll}1 & 75 & 15 & 00\end{array}$

$\begin{array}{llll}2 & 25 & 20 & 00 \\ 2 & 00 & 18 & 00\end{array}$

BIGNONIA radicans (Trumpet Creeper)...... 200

CELASTRUS scandens (Bittersweet)......... 200

CLEMATIS, Large-flowering-

Jackmanii. Purple. .

Gipsy Queen. Purple

M. Koster. Rosy pink.

Miss Bateman. White.

ville de Lyon. Crimson.

Henryi. White.

Standishii. Dark blue.

coccinea. Small flowers; coral-red.

Each $10 \quad 100$

$\begin{array}{lllllll}\$ 0 & 40 & \$ 3 & 25 & \$ 30 & 00\end{array}$

$\begin{array}{lllll}40 & 3 & 25 & 30 & 00\end{array}$

$\begin{array}{llllll}40 & 3 & 25 & 30 & 00 \\ 40 & 3 & 25 & 30 & 00\end{array}$

$\begin{array}{llllll}40 & 3 & 25 & 30 & 00\end{array}$

$\begin{array}{llllll}40 & 3 & 25 & 30 & 00\end{array}$

$\begin{array}{llllll}40 & 3 & 25 & 30 & 00 \\ 40 & 3 & 25 & 30 & 00\end{array}$

$\begin{array}{llllll}40 & 3 & 25 & 30 & 00\end{array}$

C. paniculata (Japan Clematis)

Small white flowers.

2 -year, strong plants.

3 -year, strong plants.

$\begin{array}{llll}1 & 20 & 10 & 00 \\ 1 & 75 & 15 & 00\end{array}$

HONEYSUCKLE (Lonicera)

Hall's Japan

Heckrottii. Red

Scarlet Trumpet

Yellow Trumpet.

$40 \quad 3 \quad 25$

$\begin{array}{lll}3 & 25 \\ 3 & 25\end{array}$

3 .

3000

MATRIMONY VINE

$175 \quad 1500$

WISTARIA

Purple. Heavy................. $65 \quad 600$

White.

$\begin{array}{lll}65 & 6 & 00\end{array}$ 


\section{Ornamental Deciduous Trees}

AILANTHUS (Tree of Heaven). ASH

6 to $\delta$ feet.......................

American. 8 to 10 feet................. $50 \quad 450$

10 to 12 feet.................... $85 \quad 750$

\section{CATALPA}

speciosa. 4 to 6 feet.

10 to 12 feet.

Bungei (Chinese Catalpa).

2 -year-old .................. 150

CRAB, Bechtel's Double-flowering. 3 to $t$ feet........................... 75

ELM

American. 6 to 8 feet

$\delta$ to 10 feet....

10 to 12 feet.............

12 to 14 feet.

GINKGO. 6 to 8 feet

HACKBERRY (Nettle Tree).

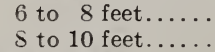

JUDAS TREE (Red-Bud)

4 to 6 feet...

\section{LINDEN}

American (Basswood).

6 to 8 feet.....

$s$ to 10 feet.
MAPLE

Norway. 6 to 8 feet.

Each Per 10

.

Wier's Cut-leaved.

8 to 10 feet........................ $50 \quad 500$

10 to 12 feet................... $100 \quad 900$

MAPLE

Schwedleri (Purple-leaved).

6 to 8 feet...................... $100 \quad 900$

8 to 10 feet..................... $175 \quad 1500$

MULBERRY

Russian. 6 to 8 feet.................. $30 \quad 250$

POPLAR

Carolina. 8 to 10 feet, 1 - to $1 \frac{1}{4}$-inch caliper ... $\quad 25 \quad 190$

10 to 12 feet, $1 \frac{1 / 4}{-}$ to $1 \frac{1}{2}$-inch caliper ........ $45 \quad 350$

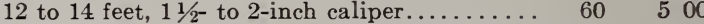

TULIP TREE. 6 to 8 feet............... $50 \quad 450$

\section{TREES OF DROOPING HABIT}

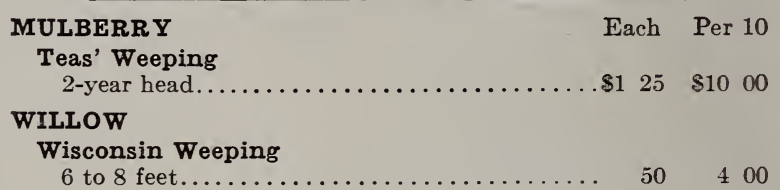

\section{Fruit Trees and Small Fruit Plants}

\section{SELECTED FRUIT TREES}

Each Per 10

APPLES. 5 to 6 feet, $11-16$ inch caliper .......... $\$ 0 \quad 30 \quad \$ 2 \quad 50$

Early Varieties-

Early Harvest

Duchess of Oldenburg

Fall and Winter Varieties-

Baldwin

Ben Davis

Fameuse

Gano.

Grimes' Golden

Rome Beauty

CRAB AFPLES

Hyslop
Whitney

Red June

Maiden's Blush

Yellow Transparent

Northern Spy

Jonathan

Tolman Sweet

Wealthy

Winesap

$\begin{array}{llll} & & & \end{array}$

Florence

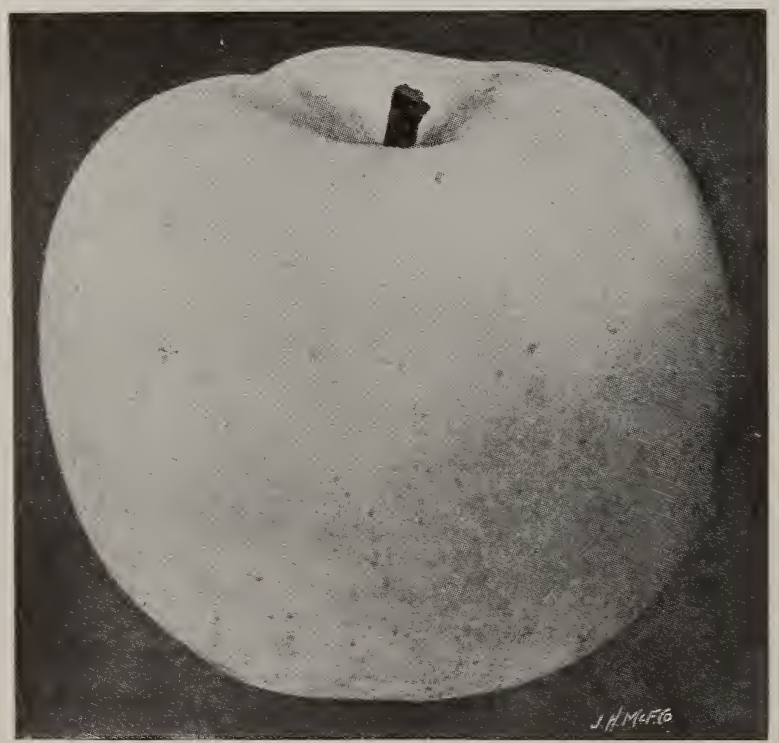

GRIMES' GOLDEN APPLE
SELECTED FRUIT TREES, continued

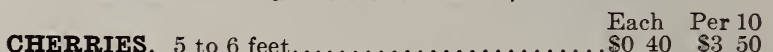

Early Richmond

Montmorency

Sweet Varieties.

Black Tartarian

Windsor

$50 \quad 400$

$25 \quad 200$

PEACHES. 5 to 6 feet.

Barnard's

Elberta

Crawford's

PEARS. 5 to 6 feet.

Bartlett

Flemish Beauty

Kieffer

PLUMS. 5 to 6 feet

Abundance

Burbank

Satsuma

Champion

Oldmixon Free

Yellow St. John

Garber

Seckel

Laurence

Lombard

Wild Goose

Shropshire Damson

\section{SMALL-FRUIT PLANTS}

BLACKBERRIES

Early Harvest

Erie

Per 10 Per 100

RASPBERRIES

Cumberland, black

Cuthbert, red

Gregg, black

CURRANTS. 2-year-old

Black Champion

Cherry

so 30 s2 100

Snyder

GOOSEBERIES. 2-year-old.

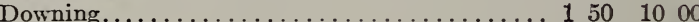

Houghton...................... 1501000

Red Jacket.......................... $200 \quad 1500$

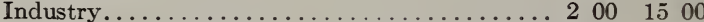

GRAPES. 2-year-old

Brighton

Concord

Niagara

Plum Farmer, black $40 \quad 250$

Kiansas, black

$\begin{array}{ccc}100 & 700\end{array}$

Wilder

Whiter Grape

Moore's Early

STRAWBERRIES

Brandywine

Climax

Senator Dunlap

Steven's Late Champion

Per 100 Per 1,000

Wm. Belt 


\section{Alphabetical Price-List of Florists' Sundries}

\section{UNLESS OTHERWISE STATED, THE PURCHASER PAYS THE TRANSPORTATION CHARGES}

Prices are subject to market changes in value. This particularly applies to Fertilizers, Wire, Wire Stakes, etc.

APRONS, Rubber. For use in potting or watering. Made of best-quality material. Medium weight, 34 in. wide, 46 in. long, weighs about 2 lbs. 4 ozs., each $\$ 2$. Single-coated, light-weight, 26 in. wide, $36 \mathrm{in}$. long, each $60 \mathrm{cts}$.

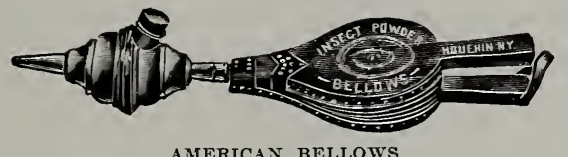

AMERICAN BELLOWS

BELLOWS, American. For applyiñg insecticides, sulphur, etc. 20 in. over all, $51 / 2$ in. wide, 4 -oz. cap, each $\$ 1.25$.

24 in. over all, $71 \%$ in. wide, 8 -oz. cap, each $\$ 1.50$.

Metal receivers only, for Bellows, 4 oz., each 60 cts.; 8 oz., each $75 \mathrm{cts}$.

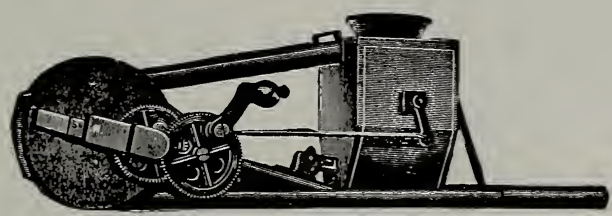

CHAMPION POWDER GUN

\section{Dusters}

Champion Powder Gun. For applying sulphur, bordeaux paris green and other dry insecticide powder. A very convenient machine where large areas are to be dusted. Dusts one or two rows at a time. Weighs about $6 \mathrm{lbs}$, weight packed, $17 \mathrm{lbs}$ Each machine has four 16-in. tubes, six nozzles, two straps; reservoir holds one quart of powder. Each \$8.50.

Dry Powder Duster No. 12. A serviceable article where only a few plants are to be treated. Made of heavy tin, capacity one and one-half quarts. Each 70 cts.

Jumbo. A convenient tin Duster of peculiar shape for holding in the hand. Desirable for treating individual plants. 4 in. in diameter, capacity about four ounces. Each $25 \mathrm{cts}$.

Peerless Sulphur Blower. Most effective, rapid and economical way of applying sulphur or any powder. A 200 -foot rose-house can be powdered evenly in ten minutes. Complete, each $\$ 4$.

\section{Lily Bowls}

For the culture of Hyacinths, Narcissi, etc.

Iridescent Glass, or Rubigold. An imitation cut-glass vase with a rubigold finish; grape pattern. Diameter 7 in. Each 20 cts., doz. $\$ 2$.

Imitation Cut-Glass. Of neat pattern. Diameter 8 in. Each 20 cts., doz. $\$ 2$.

Earthenware. Handsomely glazed. Diameter 6 in. Each $10 \mathrm{cts}$. doz. $\$ 1$.

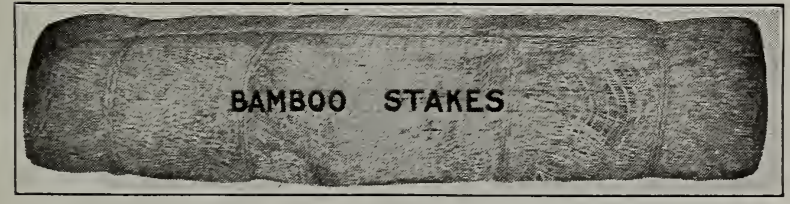

\section{Bamboo Stakes}

Strong, light, durable, for supporting plants. Packed 2,000 to the bundle. $6 \mathrm{ft}$., $100,85 \mathrm{cts}$.; $500, \$ 3.75 ; 1,000, \$ 6.50$.

Japanese Bamboo. Dyed green. Light, strong and lasting.

$1 \mathrm{ft}$. $100 \quad 1,000$

$11 / 2 \mathrm{ft}$.

$21 / 2 \mathrm{ft}$

$3 \mathrm{ft} . \mathrm{ft}$.

$4 \mathrm{ft} . .$.

$\begin{array}{llll}25 & \$ 2 & 00 \\ 35 & 3 & 00\end{array}$

$\begin{array}{llll}50 & 4 & 35 \\ & 4 & 35\end{array}$

$\begin{array}{llll}50 & 4 & 35 \\ 60 & 5 & 00\end{array}$

$\begin{array}{lll}60 & 5 & 00 \\ 70 & 6 & 25 \\ & 7 & 0\end{array}$

$80 \quad 7 \quad 00$

CARNATION BANDS, Rubber. Thin rubber bands to be slipped over the bud just before the petals unfold, or used on open flowers with burst calyxes. $1 / 4 \mathrm{lb}$. $\$ 1,1 \mathrm{~b} . \$ 3$, postpaid.

CARNATION STAPLES, Pillsbury's. 1,000 Carnations saved for 50 cts. This Staple is a very fine green wire and nearly invisible, giving the flower a perfect appearance. 1,000, $50 \mathrm{cts}$.
CAPE FLOWERS. White. Extra select, lb. $\$ 1.35$; select, lb. $\$ 1.10$. Can supply Cape Flowers in almost any bright shade at $\$ 1.35$ per lb.

CótTON WADDING. For packing flowers. White. Dozen sheets 50 cts., bale; 480 sheets, $\$ 12.50$.

CYCAS LEAVES, Preserved. Perfect in shape and natural green in color. Imported stock; best quality. Length
8 to 12 in
12 to $16 \mathrm{in}$
16 to 20 in
20 to 24 in.
24 to 28 in
28 to 32 in
32 to 36 in.
36 to 40 in....
40 to 44 in....

\begin{tabular}{|c|c|c|}
\hline tach & Doz & 100 \\
\hline $\begin{array}{lll}0 & 05\end{array}$ & & $\begin{array}{lll}\$ 3 & 00\end{array}$ \\
\hline 06 & 50 & \\
\hline 07 & 6 & \\
\hline & $\begin{array}{l}8 \\
9\end{array}$ & 550 \\
\hline 14 & 10 & \\
\hline $1:$ & & \\
\hline $\begin{array}{l}15 \\
18\end{array}$ & 175 & $\begin{array}{lll}9 & 50\end{array}$ \\
\hline
\end{tabular}

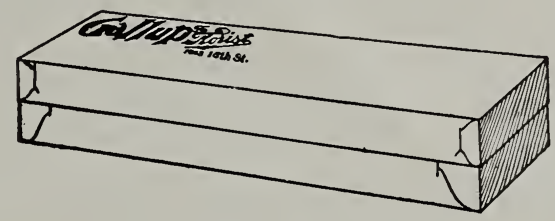

\section{Cut-Flower Boxes}

We can fill orders for plain Cut-Flower Boxes promptly, as we carry a large stock. Palm-green boxes only are carried in stock Mist-Gray and Mist-Brown shipped with slight delay. If, however you wish your boxes printed, this will delay your order ten days to two weeks. Printing in one-color ink, $\$ 1$ per 1,000 additional; in gold or silver, $\$ 2$ per 1,000 additional.

\section{Palm-Green, Mist-Brown or Mist-Gray}

The quality of these boxes is unexcelled. All boxes are made from moisture-proof material and stock runs uniform in color.

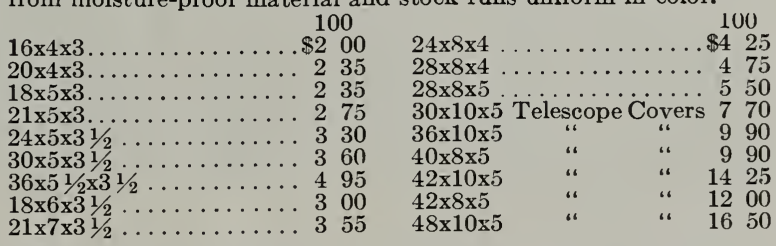

Extra-Heavy Spray and Design Boxes, PalmGreen

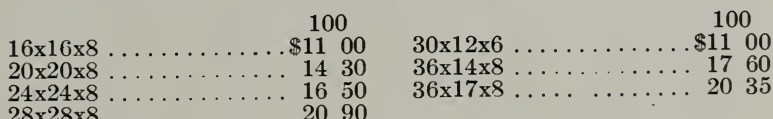

$28 \times 28 \times 8$

2090

Violet Boxes, Palm-Green, Mist-Brown or Mist-Gray

$7 \times 4 \times 4$ 100 $\$ 275$ $10 \times 6 \times 5 \frac{1}{2}$
$12 \times 8 \times 5 \frac{1}{2}$

DIBBER. For setting plants.

Wood handle, steel point, each 30 cts. All-Iron Dibber, each 25 cts.

\section{Doves}

Of these we have a splendid assortment, perfect in form and of best quality. Wings can be bent up or down to suit fancy. Please state head position when ordering. These Doves are mounted on stands, and particular attention is given to packing.

No. 1. Fancy. Blue label. Each $\$ 1.40$.

No. 2. Fine. Red label. Each, \$1.15.

FASTENERS, Eureka. A clip to attach the carnation stakes to cross-wires. Per 1,000, $\$ 1.50$.

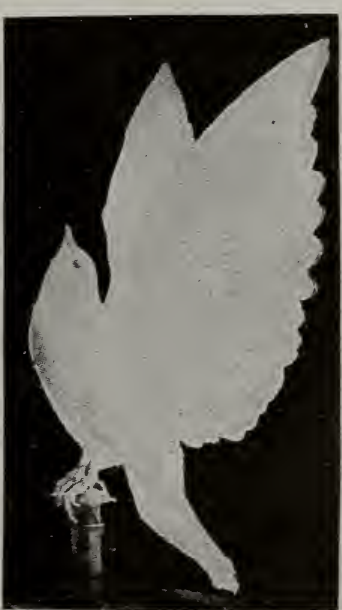




\section{Fertilizers}

ASHES, Unleached Hardwood. Mellows and sweetens hard, sour soils. Especially beneficial to cabbage and onions, all farm crops and lawns. Absolutely odorless. 25 lbs. $50 \mathrm{cts}$., 50 lbs. 85 cts., 100 lbs. $\$ 1.35,500$ lbs. $\$ 5,1,000$ lbs. $\$ 9.25$, ton $\$ 18$.

BLOOD AND BONE. This stimulates root-action and improves the color of flowers. Use it for soil in Carnation benches, to mix with potting soil and as quick-acting fertilizer for all vegetables. One or two quarts to a bushel of soil. One to two tons to the acre for garden plots. In rows or drills, two hundred pounds to the acre. 25 lbs. $70 \mathrm{cts}$., 50 lbs. $\$ 1.15,100 \mathrm{lbs} . \$ 2,200 \mathrm{lbs}$. $\$ 4,500$ lbs. $\$ 9,1,000$ lbs. $\$ 18$, ton $\$ 34$.

BLOOD, BONE AND POTASH. This is an all-round highgrade fertilizer, with a guaranteed analysis of ammonia 3 per cent, available phosphoric acid 8 per cent, potash 1 per cent. It is especially desirable for market-gardeners, florists and general garden use, and an excellent potato fertilizer. 25 lbs. general garden use, and an excellent potato fer
95 cts., 50 lbs. $\$ 1.75,100$ lbs. $\$ 3,200$ lbs. $\$ 5.50$.

BONE MEAL. Absolutely Highest Grade. It is ground fine and is an excellent top-dressing for lawns, grass lands, farm and field crops, also for mixing with potting soil. 5 lbs. 25 cts. 10 lbs. 40 cts., 25 lbs. 70 cts., 50 lbs. $\$ 1.25,100$ lbs. $\$ 2.15$, bag of 200 lbs. $\$ 4,500$ lbs. $\$ 9,1,000$ lbs. $\$ 18$, ton $\$ 34$.

BOWKER'S PLANT FOOD. Made expressly for flowers grown in the house or garden; clean, odorless, and produces early, abundant flowers, greatly adding to the richness and brilliancy of color. Stimulates the growth. Small size, each $15 \mathrm{cts}$., doz. of color. Stimulates the growth. Small size, each $15 \mathrm{cts}$., d
$\$ 1.50$; large size, each 25 cts., doz. $\$ 2.50$, box of 24 for $\$ 4.50$.

CATTLE MANURE, Shredded. Pure, high-quality Cattle Manure in most concentrated and effective form. Supplies carefully selected from brick-paved cattle-pens only. No mixture of other manures. Reduction process takes out the bedding and kills fungi and germs that make trouble in the greenhouse. More economical than stable manure; convenient to keep a stock always on hand for use at best time; easily handled and applied; unequaled for bench and potting soil, liquid manuring and topdressing. Shredded Cattle Manure is packed direct from the dryers, and is comparatively coarse and rough; suitable for mulching, etc. 100 lbs. $\$ 1.60,500$ lbs. $\$ 6,1,000$ lbs. $\$ 9.50$, ton $\$ 18$.

CATTLE MANURE, Pulverized. Same material as the Shredded passed through grinders to further reduce it to a finely pulverized product. 100 lbs. $\$ 1.65,500$ lbs. $\$ 6.25,1,000$ lbs. $\$ 10$, ton $\$ 19$.

DRIED BLOOD. Splendid nitrogenous fertilizer for Roses, Carnations, 'Mums, Potted Plants, etc. 5 lbs. 40 cts., 10 lbs. $70 \mathrm{cts}$., 25 lbs. $\$ 1.45,50$ lbs. $\$ 2.25,100$ lbs. $\$ 4.25,200$ lbs. $\$ 8.25,500$ lbs. $\$ 19.25$.

HORN SHAVINGS. A valuable and rich fertilizer, used to mix with soil for vases and plant-tubs. 5 lbs. $40 \mathrm{cts}$., $10 \mathrm{lbs} .70 \mathrm{cts}$., 25 lbs. $\$ 1.25,100$ lbs. $\$ 4.50$.

KAINIT. Contains about $121 / 2$ per cent potash. Apply at rate of 1,000 lbs. to the acre. Excellent for either lawn or vegetablegarden.

LAND PLASTER. Particularly valuable as a top-dressing. Apply at the rate of 25 to 50 lbs. to 1,000 square feet. 50 lbs. $65 \mathrm{cts}$, 100 lbs. $\$ 1$, bbl. (about 300 lbs.) $\$ 2.50$.

LEAF-MOLD. For florists' use. Bag $\$ 2.25,6$ bags $\$ 12$.

MURIATE OF POTASH. A high-grade potash containing about 45 per cent. Apply at the rate of about 1 to $10 \mathrm{lbs}$. to 1,000 square feet. Should be used with considerable care, as it is a strong chemical fertilizer.

NITRATE OF SODA. A quick-acting fertilizer for all crops, containing about 16 per cent nitrogen. Can be applied at the rate of $1 \mathrm{oz}$. to 2 gallons of water. $10 \mathrm{lbs} .65 \mathrm{cts} ., 25 \mathrm{lbs}$. $\$ 1.45,50 \mathrm{lbs}$. $\$ 2.75,100$ lbs. $\$ 5$.

PEAT, Orchid. (Fern-root.) Carefully cleaned by hand, and of the highest quality. Bbl. \$2.25. Azalea Peat. A well-rotted potting material for mixing with heavy soil. Bbl. \$2.25. (Peat is shipped a barrel to a sack to save transportation charges.)

SHEEP MANURE, Pulverized. Carefully selected and treated to destroy weed seeds and produce a highly concentrated, reliable fertilizer for home use. This is the strongest and best natural fertilizer for lawn and gardens, vegetable- and fruit-growing, and is quick-acting and safe. Valuable for mixing with potting soil and top-dressing. 25 lbs. 60 cts., 50 lbs. $\$ 1,100$ lbs. $\$ 1.75,500$ lbs. $\$ 7.50,1,000$ lbs. $\$ 12$, ton $\$ 22$.

SULPHATE OF AMMONIA. Rich in nitrogen. 5 lbs. (for 250 square feet of surface soil) 65 cts., 25 lbs. $\$ 2,100 \mathrm{lbs}$. $\$ 7.50$.

SULPHATE OF POTASH. About 48 per cent of potash. Good for potatoes or small fruits.

VEGETABLE AND LAWN FERTILIZER. An all-round fertilizer for the vegetable-garden and for the lawn. Is a rich, wellbalanced fertilizer, with a guaranteed analysis of ammonia 3 per cent, available phosphoric acid 8 per cent, potash 1 per cent, and is used at the rate of about 25 lbs. to 1,000 square feet. Can be used as a top-dressing for old lawns, light applications giving better results than one heavy application. 25 lbs. $95 \mathrm{cts}$., 50 lbs. $\$ 1.75,100$ lbs. $\$ 3,200$ lbs. $\$ 5.50$.

\section{Fumigating Lamps}

Eureka No. 3 Fumigator. Galvanized iron. The most popular size, holding one-half bushel of stems. Each $\$ 2.50$.

Defiance. All metal. Burns kerosene. Oil-fount and cup for tobacco preparation are both drawn from one piece, so they cannot leak. No glass to break. Each $50 \mathrm{cts}$.

Nicoticide Lamp. A glass fumigator especially designed for Nicoticide, but working equally well with other preparations. Each 65 cts.

\section{Glazing Tools and Materials}

GLASS CUTTER, Diamond Universal. Each $\$ 4.50$. Glass Cutter and Glazing Tool. With straight handle, each $10 \mathrm{cts}$., doz. 90 cts.; with ball handle, each 10 cts., doz. $\$ 1$.

PUTTY BULB, Scollay's. Has a tube for applying liquid putty. Each $90 \mathrm{cts}$.

PUTTY, Twemlow's Old English. A semi-liquid Putty which can be applied with putty bulb or glazing machine. It is a most excellent Putty, especially prepared for putting the glass in sash or filling in cracks. Gal. $\$ 1.65,5$ gals. $\$ 8$.

MASTICA. A substitute for putty. It is easy to apply and is not affected by extremes of weather. One gallon covers about 290 running feet. Gal. $\$ 1.35,6$ gals. $\$ 7.65,10$ gals. $\$ 12.15$, bbl. (22 gals.) $\$ 25.25$.

MASTICA MACHINE, to apply Mastica. Each $\$ 1 . \overline{0} 0$.

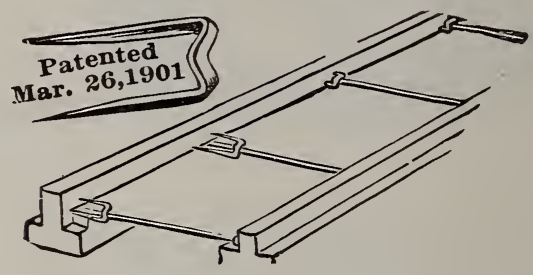

PEERLESS GLAZING POINTS

GLAZING POINTS, Peerless Perfect. No rights, no lefts, no trouble to apply. No. 1, for small, single-thick glass; No. 2 for larger, single thickness; No. $21 / 2$, for double-thick glass and skylights. Box of 1,000, 60 cts.; by mail, $80 \mathrm{cts}$. In lots of 5,000 , 55 cts. per 1,000 , not prepaid.

Pincers for setting Peerless Points in place, each $50 \mathrm{cts}$.

GLAZING POINTS, Zinc City. These Points can be driven right or left on any size or thickness of glass. The offset head makes them easy to drive or extract. and absolutely prevents glass from slipping. Made from pure zinc. Put up in one-pound pack.ages. 7/8-inch, 1,200 to lb. Lb. 50 cts., 10 lbs. $\$ 4.25$.

PEERLESS GLASS REPAIR CLAMP. A simple device for repairing broken greenhouse glass Easily adjusted from inside of house. Box of 100 clamps, $\$ 1$.

GARDEN LINE. Best hemp, braided. $100 \mathrm{ft} .65 \mathrm{cts}$.

Garden-Line Reel. Large size, each 65 cts.

GRAFTING WAX. In bars. $1 / 4$ lb. 10 cts., 1/2lb. 18 cts., lb. 30 cts. GRASS HOOKS, or SICKLES. The famous S. Marshall's "Soldier Brand," imported English Sickles, with riveted back. No. 0 each 50 cts., doz. $\$ 4.20$; No. 1 , each 55 cts., doz. $\$ 4.75 ;$ No. 2. each, 60 cts., doz. $\$ 7$.

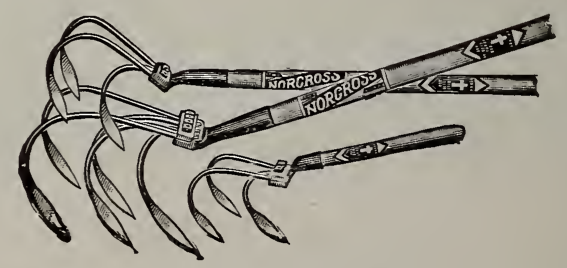

NORCROSS CULTIVATOR

HOES, Imported English. Dutch Scuffle. Best pattern. 6 -in., each $55 \mathrm{cts}$; 7 -inch, each $60 \mathrm{cts}$; 8 -inch, each 65 cts.

American Scuffie. 8-inch, cast-steel, 6-foot handle, each 65 cts. Garden. Especially high-grade, razor-steel blade, each 65 cts. solid socket, cast-steel, each 50 cts.

Warren. V-shaped blade, each $70 \mathrm{cts}$.

Weeding Hoe. Each $25 \mathrm{cts}$.

Norcross Cultivator. A very handy tool. 5-prong, each $75 \mathrm{cts}$. 3-prong, each 50c.; Midget, short handle, 3-prong, each $35 \mathrm{cts}$ HOTBED BLANKETS. Made of burlap, lined with waste, wool and cotton, quilted. 76 in. $x 76$ in., each $\$ 1.60 ; 40$ in. $x 76$ in. each $\$ 1.10$.

REED MATS. 72 in. $x 78$ in. Made of reeds or grass. Excellent for protecting hotbeds in early spring instead of glass. Each $\$ 1.75 ; 54$ in. $: 78$ in., each $\$ 1.65$. 


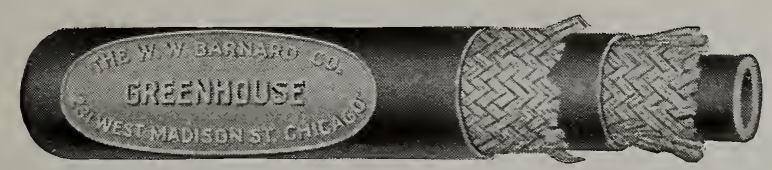

BARNARD'S GREENHOUSE HOSE

\section{Hose and Accessories}

BARNARD'S GREENHOUSE. A selected grade of molded, non-kinkable Hose, with a smooth cover. Made of strictly first-class rubber, and guaranteed against defects during a season. Hose can be supplied cut to any length, couplings included with 25 - $\mathrm{ft}$. lengths or over. $3 / 4$-inch, $25 \mathrm{ft}$. $\$ 4.25,50 \mathrm{ft}$. $\$ 7.75$. In longer lengths, per ft. $15 \mathrm{cts}$.

Revero. A light, strong, non-kinkable, molded hose, with smooth cover. $3 / 4$-inch, in lengths over $25 \mathrm{ft}$., $17 \mathrm{cts}$. per $\mathrm{ft}$., couplings included.

Florists' Brand. An extra-quality woven Hose, particularly adapted to high pressures. $3 / 4$-inch, with couplings, $50 \mathrm{ft}$. $\$ 6.50$ $100 \mathrm{ft}$. $\$ 12$.

Gardeners' Brand. A serviceable, low-priced hose. 3/4-inch, $25 \mathrm{ft}$. $\$ 2.75,50 \mathrm{ft}$. $\$ 5,100 \mathrm{ft}$. $\$ 9$.

HOSE CLAMPS, Brass. Strong and reliable. Easily adjusted. Each 5 cts. doz. 50 cts.

Galvanized Iron. Each 3 cts., 2 for 5 cts., doz. 25 cts.

HOSE NOZZLES, Adjustable. For throwing a solid stream or a fine mist. For $3 / 4$-inch hose. Each 50 cts.

Boston Sprinkler, Brass. For $3 / 4^{-i n c h}$ hose. State whether coarse or fine spray is wanted. Each $50 \mathrm{cts}$.

Wittbold. The spray is fan-shaped and can be changed instantly from a fine mist into a heavy stream, by moving the slide that is controlled by the hand which holds the hose. Each \$1.
HOSE COUPLINGS. Spun Brass. $3 / 4$-inch. Per set $20 \mathrm{cts}$.

Nickeled. $3 / 4$-inch. Per set 25 cts.

"The Snap." This is a new Coupler similar to the well-known Acme. It has a stationary washer - a very great convenience. No. 1, screws onto hydrant or old threaded coupling, each 20 cts., doz. \$2. No. 2, screws into a faucet or ground pipe, each 15 cts., doz. $\$ 1.50$. No. 3 , gooseneck, screws into old threaded coupling, each 15 cts., doz. $\$ 1.25$. No. 4 , corrugated, for insertion directly into hose, each 12 cts., doz. $\$ 1$. No. 5 , corrugated, use with No. 4 in connecting hose, each $20 \mathrm{cts}$., doz. $\$ 2.25$.

HOSE MENDERS, Cooper's. Made of brass. Will not rust or wear out. Does not reduce the diameter of the hose. Fiasily adjusted without special tools. For $1 / 2$-inch and $3 / 4$-inch hose. Each 8 cts., 4 for 25 cts., doz. 75 cts.

Iron. $3 / 4$-inch. Each 5 cts., doz. 50 cts.

Wood. $3 / 4$-inch. Doz. 15 cts.

HOSE WASHERS. $3 / 4$-inch. Doz. $5 \mathrm{cts}$, gross $50 \mathrm{cts}$.

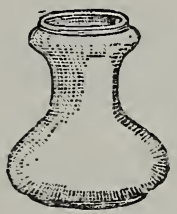

\section{Hyacinth Glasses}

Supplied in three distinct colors.

Tall. Each 20 cts., doz. $\$ 1.45,3$ doz. $\$ 4$.

Tye-shaped. A low glass, preferred by some. Each 20 cts.,
doz. $\$ 1.55,3$ doz. $\$ 4.25$.

TYE IMMORTELLES. Best Frenchgrown; our own importation. White, Scarlet, Pink, Dark Blue, Purple. Colors, bunch 40 cts., doz. $\$ 4,100 \$ 29$. Natural, bunch 35 cts., doz. $\$ 3.50,100 \$ 26$.

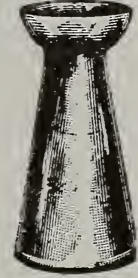

TALI

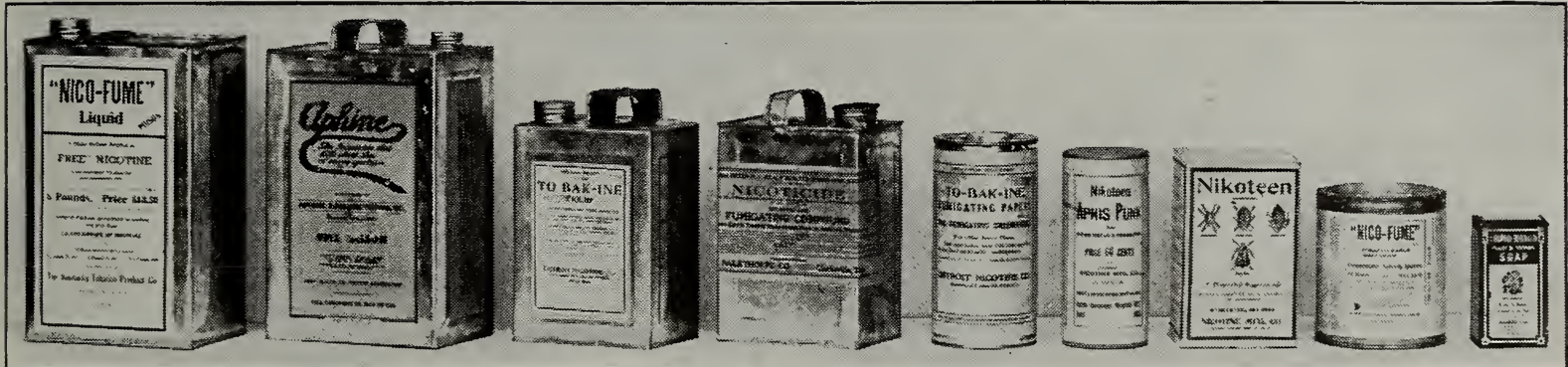

\section{Insecticides and Fungicides}

Insecticides may be grouped into two classes: First, those for such insects as aphis, scale, red spider, white fly, thrip, mealy bug, etc. These insects damage the plant by sucking the juice, and to combat this group it is necessary to apply a spray which corrodes their bodies or stops their breathing-pores. Sprays of this class must be applied so that the spray will hit the insect, as it is more effective in this way. Another method is to use some form of fumigating material and suffocate them.

The second class of insects embraces those which chew the foliage, such as the caterpillar, currant worm, cucumber beetle, etc. These must be fed a poison which they will take into their stomachs with the leaf.

Special directions will be supplied with the different spray materials for their use. Prices subject to market.

APHINE. For plant-sucking insects. Pt. 65 cts., qt. $\$ 1$, gal. $\$ 2.50$.

APHIS PUNK. Fumigating material which comes in cases, 12 air-tight boxes to a case. Each box contains 12 sheets, $6 \times 3 t$ inches; 3 sheets will fumigate a $100-\mathrm{ft}$. house. Weight of case, 12 lbs. Box 60 cts., case (12 boxes) $\$ 6.50$.

ARSENATE OF LEAD, Paste. Kills leaf-eating insects. This material is especially recommended on account of its adhesiveness to the foliage. It is not washed off by the first rain. $2 \mathrm{lbs}$. to 50 gallons of water, one tablespoonful to one gallon of water. Lb. 25 cts., 5 lbs. 85 cts., 10 lbs. $\$ 1.60,25$ lbs. $\$ 3.50$.

Dry. The dry form is a recent introduction. It has many points to recommend it. It can be used with a powder gun or mixed with water and used as a liquid spray. Equally effective as the paste form. 1/2lb. 25 cts., lb. 40 cts., 5 lbs. $\$ 2$.

BORDEAUX MIXTURE. Is the best Fungicide for curing such diseases as black-rot, mildew, scab, rust, etc., on both fruits and plants. To meet varying needs, we can supply this mirture in the following form: 1-lb. cartons, each $25 \mathrm{cts}$.

Fungi Bordo. Dry, for powder-guns. Lb. 30 cts., 5 lbs. $\$ 1.40$, 10 lbs. $\$ 2.50$

Tuber Tonic. A dry bordeaux and paris green. 4 to 6 pounds to 50 gallons of water. Lb. 35 cts., 5 lbs. $\$ 1.75$.

"Lion Brand," Semi-Liquid. Mix 1 quart with 49 gallons of water. Pt. 25 cts., qt. 40 cts., gal. $\$ 1$.

FISH-OIL SOAP. Commonly called Whale-Oil Soap. A wash for trees and plants infested with scale and sucking insects. $1 / 2 \mathrm{lb}$. $10 \mathrm{cts}$, lb. $18 \mathrm{cts}$., 2 lbs. $30 \mathrm{cts} ., 5 \mathrm{lbs} .65 \mathrm{cts}$.
FIR-TREE-OIL SOAP. For sucking insects. Used at the rate of 1 ounce to 1 gallon of water. $1 / 2 \mathrm{lb}$. tin $25 \mathrm{cts}$., $2-\mathrm{lb}$. tin $75 \mathrm{cts}$.

FLOWERS OF SULPHUR. A Fungicide for checking mildew in greenhouses. Lb. 10c., 10 lbs. 75 c., 50 lbs. $\$ 2.50,100$ lbs. $\$ 4.50$.

GRAPE DUST. A Fungicide for destroying mildew, mold and rust on plants. Lb. carton 12 cts., 5 -lb. package 35 cts.

HELlebore, Powdered, White. For leaf-eating insects. A form much used for the currant worm, especially on fruit which is nearly ripe. $1 / 2 \mathrm{lb} .25 \mathrm{cts}$., lb. 40 cts., 5 lbs. $\$ 1.75,10$ lbs. $\$ 3.25$.

KEROSENE EMULSION, Liquid. For sucking insects. This is one of the standard remedies for such insects as scale, aphis, etc. Qt. 40 cts., gal. $\$ 1,5$ gals. $\$ 4.25$.

LIME-SULPHUR SOLUTION. For sucking insects. Qt. $25 \mathrm{cts.,}$ $1 / 2$ gal. 50 cts., gal. 75 cts., 5 gals. $\$ 3.50$.

NICOFUME. Tobacco paper for fumigating. 8 to 10 sheets required for a house 100 feet long by 20 feet wide. 24 sheets in tin can, 85 cts.; 144 sheets $\$ 4,288$ sheets $\$ 7.50$.

NICOFUME LIQUID. For spraying or fumigating. 1/20z. will fumigate 2,000 cubic feet For spraying, use one to two teaspoonfuls to a gallon of water. $1 / 4 \mathrm{lb}$. 50 cts., lb. $\$ 1.50,4$ lbs. $\$ 5.50$, 8 lbs. $\$ 10.50$.

NICOTICIDE. Fumigating compound for use in greenhouses or for spraying. For spraying, use 1 ounce to 10 to 15 gallons of water. 4 ozs. 70 cts., $1 / 2$ pt. $\$ 1.25$, pt. $\$ 2.50$, qt. $\$ 4.50$, $1 / 2$ gal. $\$ 8.25$, gal. $\$ 15$.

Special fumigators to use with Nicoticide, each $65 \mathrm{cts}$. 


\section{INSECTICIDES AND FUNGICIDES, continued}

NIKOTEEN. A fumigating and spraying material for sucking insects. $13 / 4$-oz. bottle, makes four gallons of spray, $40 \mathrm{cts}$. $1 / 2$ lb. 85 cts. (40 per cent), lb. $\$ 1.50,4$ lbs. $\$ 7$ (40 per cent strength), 8 lbs. $\$ 13.33$.

BLACK LEAF 40. Especially prepared nicotine for outdoor spraying. "Black Leaf 40 " is highly recommended by experiment stations and by spraying experts throughout the entire United States. 1 oz. (makes 4 to 6 gals.) 25 cts., $1 / 2$ lb. 75 cts., 2 lbs. $\$ 2.50,10$ lbs. $\$ 10.75$.

TOBAKINE FUMIGATING PAPER. For quick and effective fumigation. 24 sheets $\$ 1,144$ sheets ( 6 cans) $\$ 4.50,288$ sheets (12 cans) \$8.

PARIS GREEN. The old standard for chewing insects. Can be used either dry or in solution. Prices subject to market changes. $1 / 2$ lb. 22 cts., lb. 40 cts., 5 lbs. $\$ 1.75$.

SLUG-SHOT. A fine powder. Kills every garden pest, but is harmless to human life. Especially recommended for use on tomatoes, cabbage, currants, etc. Lb. cartons $15 \mathrm{cts}$, lb. cannisters, with shaker top, 25 cts., 5 -lb. pkg. 40 cts., 25 lbs., 5 cts. per lb.; bbl. (in 5-lí. pkgs.), about $300 \mathrm{lbs}$., per lb. $4 \frac{1}{2} \mathrm{cts}$. Slug-Shot Duster. 1/2gal. size, each $35 \mathrm{cts}$.

Acre-an-Hour Duster. Each $60 \mathrm{cts}$.

SULPHO-TOBACCO SOAP. For sucking insects on roses, etc. as well as on animals. 3 -oz. cake 8 cts., doz. 85 cts., 8 -oz. cake 20 cts., doz. \$2.

FUMIGATING AND DŨSTING COMPOUND. Commonly called "Tobacco Dust." A finely powdered product, especially adapted for dusting or sprinkling on flowers, plants and vegetables, or for slow fumigation. 10 lbs. 50 cts., 25 lbs. $\$ 1.10$ 50 lbs. $\$ 1.75,100$ lbs. $\$ 3.50$.

POWDERED TOBACCO. A high-grade product. $5 \mathrm{lbs} .70 \mathrm{cts}$. 10 lbs. $\$ 1,25$ lbs. $\$ 2.25,100$ lbs. $\$ 8$.

TOBACCO STEMS. Bales of 200 lbs., more or less. Per $100 \mathrm{lbs}$. $\$ 1.35$ (subject to market changes), 500 lbs. $\$ 5.75$. Special prices quoted on larger quantities.

TREE TANGLEFOOT. A sticky compound for preventing the ascent of caterpillars and all crawling insects up the trunk of trees and large shrubs. Lb. 35 cts., 3 los. $\$ 1$.

\section{Knives}

The Knives we offer are made of the best quality of steel, and are especially designed for gardeners' and florists' use.

Budding, or Propagating. No. 343, Double-bladed, each $\$ 1.25$ No. 401, Propagating, single-bladed, each \$1.35; No. 400, Budding, single blade, each \$1.50; No. 403, Propagating, longhandled, each $\$ 1.25$.

Pruning. No. 4049, single blade, rough bone handle, each $50 \mathrm{cts}$. No. 938, large blade, each $\$ 1.50$.

\section{Labels, Pot and Tree}

Pot Labels, Wooden, $5 / 8$ inch wide.

$31 / 2$-inch

4-inch.

5-inch

6-inch

10-inch

12-inch.

Garden Stakes, Wooden, 7/8 inch wide. 8-inch. 10-inch 12-inch

Tree Labels, Wooden, $31 / 2$ inches, notched and copper-wired per $1,000 \$ 1.20$.

Special prices will be quoted for large lots.

\section{Lawn Mowers}

We are agents for the Philadelphia Lawn Mower, the most widely known and satisfactory Lawn Mower made. These Mower are used in the majority of the parks and cemeteries of Chicago.

Style A, 10-inch driving wheel, 4 blades, all steel.

$$
\text { 15-inch. }
$$

21-inch

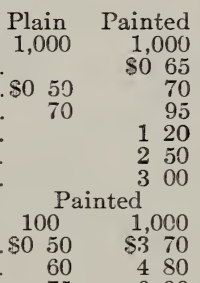

Style Graham. All steel. Highest grade of Lawn Mower.

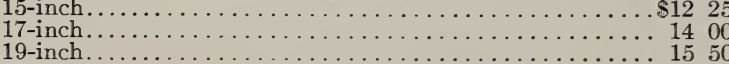

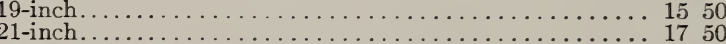

Style K. The most popular home Mower.

14 -inch.................. \$6 15 16 -inch ................................... \$6 15 18-inch..........................

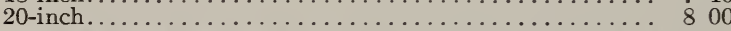

LAWN MOWER REPAIRS. For the above machines we carry repairs. We quote a discount of 10 per cent from list.

LAWN CLEANER. A practical device for removing clippings and leaves from the lawn. 24 inches wide. Each $\$ 16$.
LAWN ROLLERS, Water-weight. These Rollers are electrically welded, no rivets are used; drum cannot leak. The following sizes are the most popular. Larger or smaller Rollers can te furnished at special prices.

$\begin{array}{lcccccc}\text { Diam. } & \text { Length Sec- } & \text { Weight } & \text { Water } & \\ \text { No. } & \begin{array}{c}\text { inches } \\ \text { inches tion }\end{array} & \begin{array}{c}\text { empty } \\ \text { filled }\end{array} & \text { Each } \\ \text { WB 5 } & 18 & 24 & 1 & 75 \text { lbs. } & 300 \text { lbs. } & \$ 1040 \\ \text { WB 7 } & 24 & 24 & 1 & 125 \text { lbs. } & \text { 500 lbs. } & 1360\end{array}$

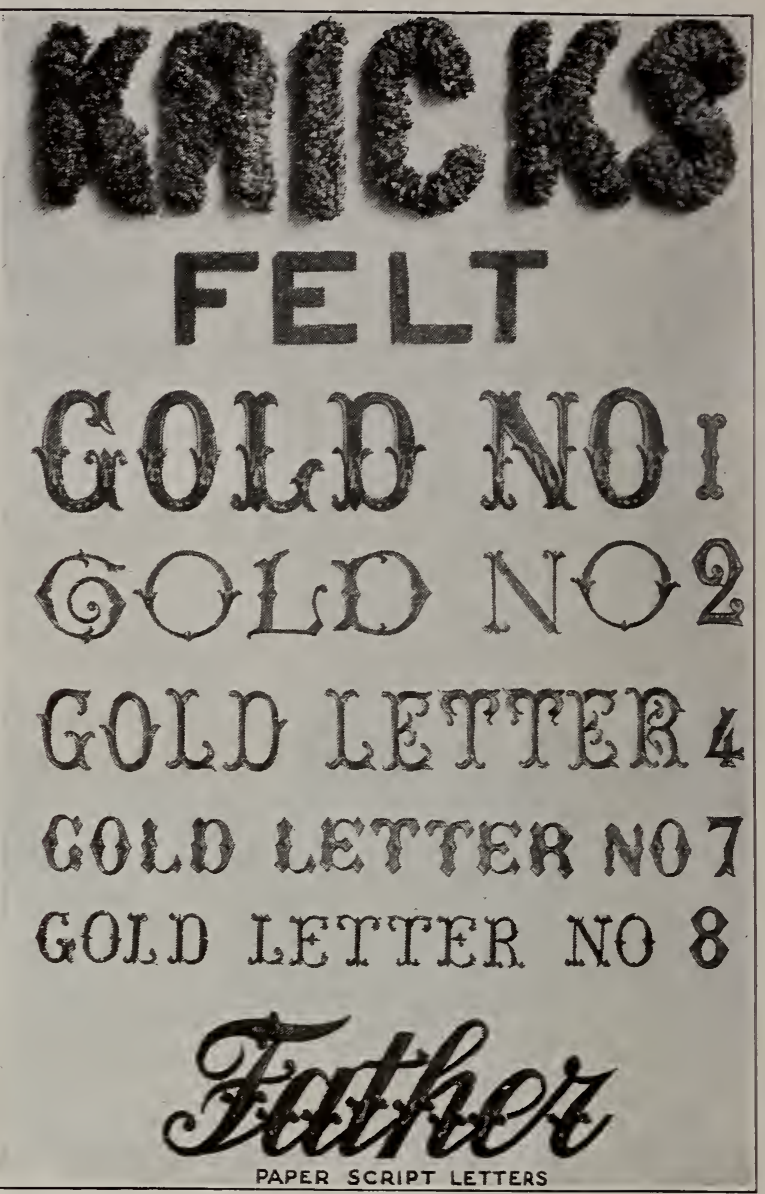

\section{Letters and Lettering}

KRICK'S IMMORTELLE LETTERS. An artistic letter of distinct merit. Purple. 2 inches. Each, 3 cts., per full 100, $\$ 2.75$. KRICK'S IMMORTELLE LETTERING. Purple. In four-yard coils. Each 90 cts., 6 packages ( 24 yards) $\$ 5.25$.

BOSTON LETTERS AND FIGURES. Similar to Krick's Letters, with patent fastener. 2 inches. Purple. Each 3 cts., per $100, \$ 2$.

BOSTON SCRIPT LETTERS. We offer the following words, made up of Boston Letters in purple. 2 inches in length. Per letter 3 cts., per $100, \$ 3$.

$\begin{array}{llll}\text { Father } & \text { Mother } & \text { Brother } & \text { Sister } \\ \text { Baby } & \text { Friend } & \text { My } & \text { Aunt } \\ \text { Husband } & \text { Wife } & \text { Our } & \text { At rest } \\ \text { Grandpa } & \text { Grandma } & \text { Darling } & \text { Dear }\end{array}$

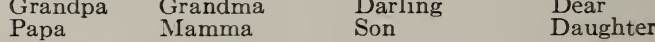

FELT LETTERS. Purple. For ribbons, chenille, etc. Very popular. Each letter gummed on the back for immediate use 1 -inch. Each $1 \mathrm{ct}$., per $100,75 \mathrm{cts}$.

PAPER LETTERS AND FIGURES. These are rich in appearance, and are considered an ideal letter for design work. Gummed on the back. The accompanying illustration shows the size and style of the letters we offer.

No. 1. Gold Each Box or

No. 1. Gold

No. 2. Gold

No. 3. Black

No. 4. Gold

No. 8. Gold

so $02 \quad 230$ $02 \quad 375$ $011 / 22$ $011 / 425$ 01210 $01 \quad 1 \quad 75$ 
LETTERS AND LETTERING, continued

PAPER SCRIPT. Quickly adjusted, low in price; gummed on back. Can be had in either purple or gold, in the same words as the Boston Script Letters. Always state kind wanted, otherwise, gold will be sent. Per inscription 5 cts.

CHENILLE. A well-known worsted, used in making letters. Carried in three sizes. Colors: White, Pink, Purple, Blue, Red

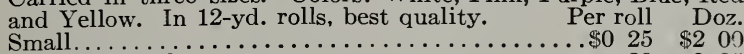

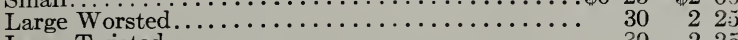

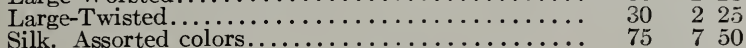

MAGNOLIA LEAVES. An article growing rapidly in public favor. Leaves keep well and are little afiected by climatic conditions. Brown shade is the most in demand. Cartons contain about 750 leaves. Colors: Brown, Green and Red, or Purple shade. Per carton, $\$ 1.50,3$ for $\$ 3.75$.

MATCH-STICKS. Pointed, heavy, hardwood sticks, either in natural color or dyed green. $\quad 1,000 \quad 1,000$ 5 -inch................. Green, \$0 $60 \ldots$. Natural $\$ 050$

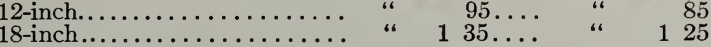

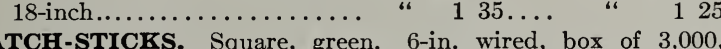
$\$ 1.25$; 6-in. unwired, box of 3,000, 75 cts.; 4 -in. wired, No. 28 wire, 4,000 , $\$ 1.50 ; 10,000, \$ 3.50$; 4 -in., unwired, 4,000, 80 cts., $10,000, \$ 2$.

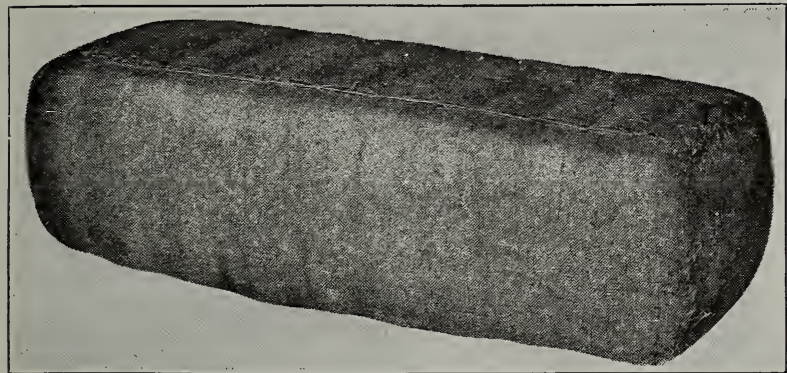

\section{Sphagnum Moss}

Select stock, put up expressly for us in large burlap bales. Quality the best. Subject to market changes. Bale $\$ 1,6$ bales $\$ 5.50$. Write for price on larger quantities. Can also supply Sphagnum Moss, packed in wire-bound bales; prices on request.

SHEET MOSS. For lining baskets, putting around pots, decorations and for general finishing. Per sack (about 2 bus.) $\$ 1.50$ two sacks for $\$ 2.75$.

MUSHROOM SPAWN, English. We import it frequently. 10 lbs. 90 cts., 25 lbs. $\$ 1.75$, 100 lbs. $\$ 6.50$.

OAK LEAVES. In Christmas colors; Bright Red, Olive-Green, Golden Brown. Very fine for decorating. Per lb. $35 \mathrm{cts}$., $10 \mathrm{lbs}$ $\$ 3$. Special prices on large quantities. Carton, $\$ 1.75$.

PLANT-TUBS, SPECIAL. Round, cedar tubs with electric welded hoops; painted green. A substantial Tub at a low price. Top $\quad$ Height

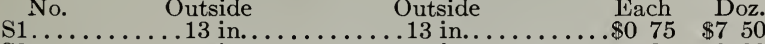

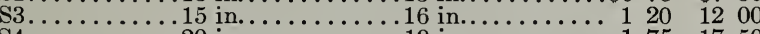

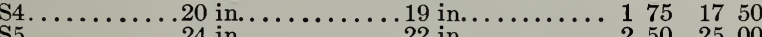
$55 \ldots \ldots \ldots \ldots 24$ in...........22 in.......... 2 $50 \quad 2500$

\section{Paper}

When ordering Paper, it is necessary for us to know width and weight. If possible, send us a sample of the desired paper with your order.

Manila Wrapping. Flat, $24 \times 36$ in. 10 lbs. $50 \mathrm{cts}$, bundle $(120 \mathrm{lbs}$. $\$ 4.50$ per $100 \mathrm{lbs}$

Manila in Rolls. Of various widths and weights. 100 lbs. $\$ 4.50$. In smaller quantity, 5 cts. per lb.

Manila Tissue. "American Beauty." Ideal paper for use with cut-flowers. Flat, $24 \times 36$ in. Ream (400 sheets) 75 cts., 10 reams $(4,000$ sheets $), \$ 6.75$.

Pure White Tissue. A high-grade paper, $24 \times 36$ in. Ream (400 sheets) $\$ 1.20$, 10 reams $(4,000$ sheets $) \$ 10$.

White Tissue. In 24-in. rolls. Per lb. 20 cts.

White Wax Tissue. $24 \times 36$ in. Per 100 sheets $50 \mathrm{cts}$., 400 sheets $\$ 1.65$.

Green Wax Tissue. A very fine quality of paper. Ream (400 sheets) $\$ 2$.

Kraft Wrapping Paper. The paper we use in our own packingroom. A tough and durable paper for heavy wrapping. In various weights and widths. Either flat or in rolls. Per lb. $7 \mathrm{cts}$., $100 \mathrm{lbs}$. or over, $63 / 4 \mathrm{cts}$. per lb.

Waterproof Crepe. Imported. Heavy. Can supply in green, pink or white. Per roll, 25 cts., doz. $\$ 2.50$.

Domestic Crepe. In various shades. Per roll $15 \mathrm{cts}$, doz. $\$ 1.50$. Rack and Cutter. For paper. Up to 36 inches. Each \$1.25.
PLANT-BED CLOTH. Made especially for gardeners' and florists' use to protect against frost or for shading purposes in summer.

Heavy grade, per yd. 25 cts.; by the piece, about 40 yds., 18 cts. Medium grade, per yd. 15 cts.; by the piece, about $60 \mathrm{yds}$., $12 \mathrm{cts}$

PLANT STAKES, Bamboo. See page 21.

\section{Plant Stakes, Galvanized Steel Wire}

Length Subject to market changes $100 \quad 1,000$ 3 feet. No. 9 wire........................ \$0 $85 \$ \$ 6 \quad 50$

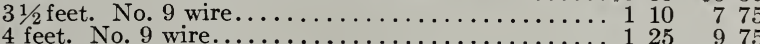

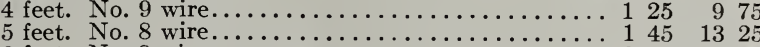

6 feet. No. 8 wire $\ldots \ldots \ldots \ldots \ldots \ldots \ldots \ldots \ldots \ldots 2001700$

Write for special prices on other sizes and larger quantities.

PLANT STARE FASTENERS. Useful for fastening stakes to the cross-wire. Per $1,000, \$ 1.50$

We call your attention to our Cane Stakes, both natural and green. We also carry for our retail trade both round and square wooden Plant Stakes, which are especially adapted for private gardens, where a neat and attractive Stake is desired. We shall be pleased to quote special prices on application.

PINS, Florists'. They have black glass heads and strong wires Put up in boxes of 1,000 .
1 -inch...........\$0 $\$ 50$
$11 / 2$-inch...............
2 -inch................ 100

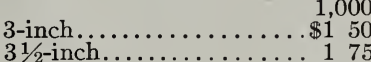
$31 / 2-$ inch
$\$ 150$

PINS, Greening. They save valuable time in greening designs. A pound contains from 1,000 to 1,100 Pins. Lb. 20 cts., 10 lbs. or over, $15 \mathrm{cts}$ per lb.

PEIRCE PAPER POTS. A waterproof Paper Flower Pot. Best thing of th kind ever made.
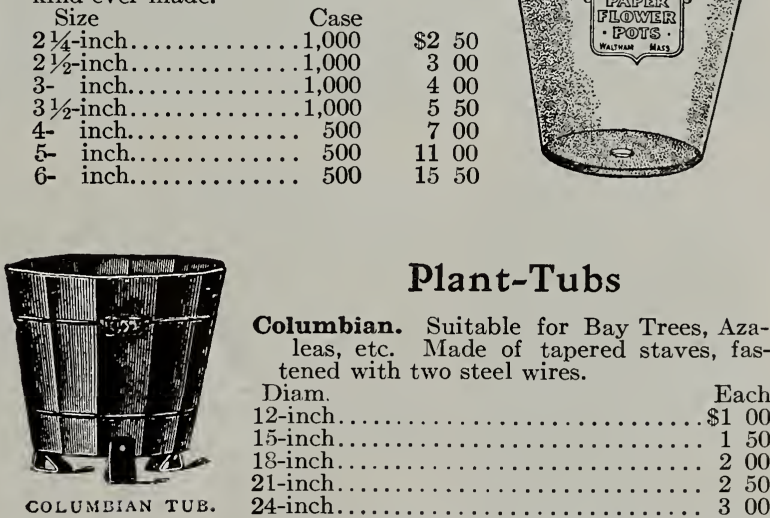

Toledo. Made of virgin red cedar stock. Strong and substantial. Hoops electric welded. Painted green.

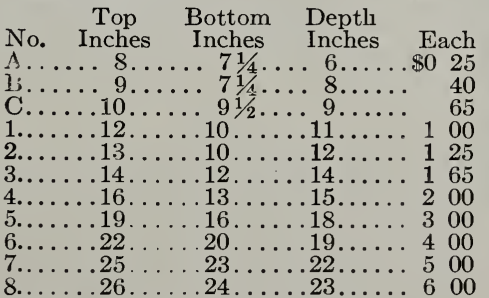

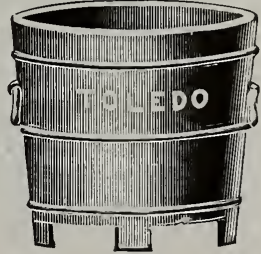

PEAT, Orchid. See Fertilizers.

RAFFIA. We have selected East Coast Raffia, which is particularly adapted to florists' use. Natural, lb. 25 cts.; Dyed, lb. $65 \mathrm{c}$.

\section{Ribbons for Florists}

While we list only the best grade of satin taffeta Ribbon, we can secure for you almost any grade, and shall be pleased to have you send us samples for prices. These Ribbons are put up in bolts of about ten yards. We do not sell less than one bolt. When ordering, mention number, width and color.

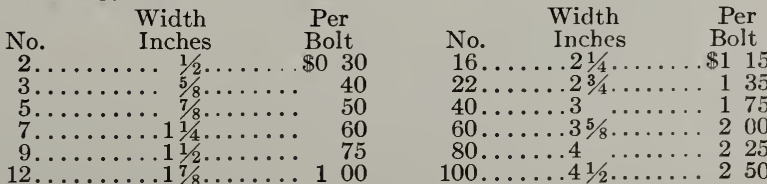

BABY RIBBON, in any color, 50-yard spool, $35 \mathrm{cts}$ 


\section{Chiffon Ribbons}

We offer both plain and dotted Chiffon in White, Pink, Lavender and Purple. Kindly state, when ordering, whether plain or dotted is desired, and the color. As a piece of Chiffon varies in length, from 30 to 45 yards, we bill you for the quantity sent. Not less than one bolt of a color soid.

Plain. Beautiful and inexpensive decorating material. 4 inches wide, per yd. 4 cts.; 6 inches wide, per yd. 5 cts.

Dotted. The dotted background of this Chiffon produces a pleasing effect. 4 inches wide, per yd. $51 / 2 \mathrm{cts}$; 6 inches wide, per yd. $61 / 2$ cts.

RUSCUS. For Christmas decorations; very pleasing and popular. Red. Rich, bright shade. Lb. 75 cts., 10 lbs. $\$ 7$.

Green. Splendid foliage color. Lb. 85 cts.

White. Lb. $\$ 1$.

Purple. Yellow and Lavender. Lb. $85 \mathrm{cts}$.

SCYTHES. Best English. For lawn and for general use. 32-, 34 - and 36 -inch, each $\$ 1.40 ; 40$-inch, each $\$ 1.50$.

SCYTHE SNATHES. Patent fastener. Each, $\$ 1.25$.

SCYTHE STONES, Talacre. Each, 25 cts., doz. \$1.75.

\section{Shears}

Border. For cutting grass around beds and shrubbery. 9-inch blade, with wheel, each $\$ 2.50$; without wheel, each $\$ 2.25$.

Lawn. With blades parallel to the lawn, for clipping grass where mower will not reach. With two wheels. 10-inch blade, each $\$ 2.50 ; 12$-inch blade, each $\$ 3$.

Hedge. The best English steel. For trimming hedges and for heavy cutting. 9 -inch blades, each $\$ 1.65 ; 10$-inch blades, each $\$ 1.90 ; 12$-inch blades, each $\$ 2.50$.

Grass. Highest-grade steel, each $\$ 1.25 ; 7$-inch, razor steel, each $\$ 1$.

Pruning. Imported. No. 205, all steel with brass springs. Shears we recommend. $81 / 2$-inch, $\$ 1.45 ; 9$-inch, $\$ 1.60$. With spiral steel spring, No. $200,81 / 2$-inch, $\$ 1.25$; No. $210,91 / 2$-inch, $\$ 1.30$; No. $190,7 \frac{1}{2}$-inch, $\$ 1.10$.

Wire Snips. For cutting annealed wire. Each 75 cts.; No. 12, forged steel snips, each $\$ 1$.

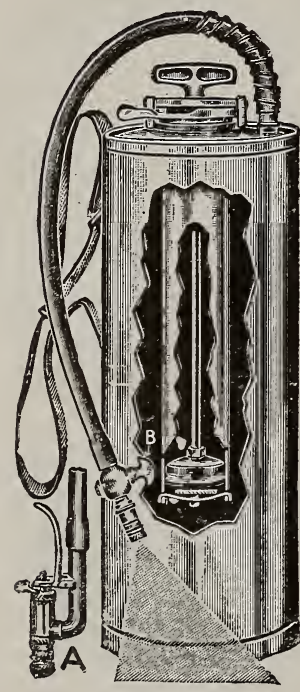

\section{Sprayers}

Auto-Spray No. 1. Most widely used compressed-air Sprayer on the market. Is the strongest and most simple in its working parts of almost any compressed-air Sprayer. Can be used for spraying insecticides, fungicides, etc. Each Sprayer is equipped with carrying strap, 2 feet of $1 / 2$-inch hose, fine spray nozzle, solid stream hose, fine spray nozzle, solid stream
cap, and shut-off. Capacity $31 / 2$ gallons. Shipping weight $15 \mathrm{lbs}$.

No.1A. Brass tank, with stop- Each ock. 1A. Brass tank, with stop- $\$ 700$ No. 1 B. Brass tank, with auto-

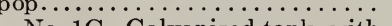

top-cock................. 475 No. 1D. Galvanized tank, with

auto-pop................. 550

Extension pipe, $45 \mathrm{cts}$. Elbow, $30 \mathrm{cts}$ Brass strainer, $\mathrm{s0} \mathrm{cts}$.

The Auto-Spray is equipped with the only non-clogging nozzle manufactured. We accomplish this feature by a patented screen arrangement. Every particle of the chemicals which would otherwise clog the nozzle is stopped by a screen.

Simplex. A compressed-air Sprayer, with the pump separate from the tank. Can be used for the same purpose as the Auto-Spray. Each is complete with a single auto-nozzle. No. 1, galvanized, $\$ 4.50$. Double nozzle, $\$ 1.50$ extra.

Binks' No. 22. Compressed-air Sprayer, 3-gal. steel tank; well made; highly recommended. Each $\$ 6.25$.

SUCCESS BUCKET PUMP. Particularly high-grade sprayer for use in a bucket. All brass, with bordeaux nozzle. Complete, each $\$ 3.75$.

Kinney. For spraying liquid manure. Each $\$ 2$.

No. 7, Lightning. Single tube; tin. Each $35 \mathrm{cts}$.

No. 20, Glass Jar Sprayer. Each $50 \mathrm{cts}$.

No. 1 Tin, Double Tube. Each 35 cts., doz. $\$ 2.75$.

No. 3, Tin Single Tube. Each 35 cts., doz. $\$ 3$.

Auto 37B. Continuous Sprayer, which will handle all solutions. Tank made of galvanized iron or brass, with tin pump. Each 1 qt.

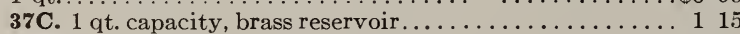

37E. 2 qts. capacity, brass reservoir.............. 150

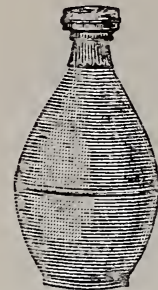

SCOLLAY'S

\section{Sprinklers}

SCOLLAY'S. Made of one piece of soft rubber, with perforated, detachable, screw cap.

Large. No, 1, 6 inches. .......... Each

Medium. No, 3,5 inches...................... 65

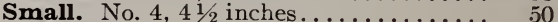

Angle-Neck. 6 inches............... 90

ELGIN. A very efficient Sprinkler, equipped with shield, which controls the direction in which the spray is thrown. Each $55 \mathrm{cts}$.

We have many other styles of Lawn Sprinklers for various purposes. We shall be pleased to answer inquiries and give prices on application

STATICE. Plain, lb. 60 cts.; colored, lb. $\$ 1$.

\section{Thermometers}

These we do not send by mail.

Hotbed or Mushroom. Wood frame; pointed brass bottom for plunging. Best, each $\$ 2$; medium, each $\$ 1.10$.

Japanned Tin Case. 8 in. Each 12 cts., doz. $\$ 1.20$.

Long-Distance. This instrument has very large figures and red fluid. Each $15 \mathrm{cts}$.

Long-Distance, Copper. Each $50 \mathrm{cts}$.

Self-Registering. Indicates highest and lowest temperatures; 8 -in.; porcelain face. Including magnet, each $\$ 2.75$; with wood face, each $\$ 2.50$.

\section{Tinfoil}

Subject to market changes.

Our Tinfoil is packed for us by one of the leading manufacturers of the country. One pound will cover approximately 2,000 square inches.

Medium. 5 or 7 inches wide. Lb. 15 cts., 2 lbs. 25 cts., 10 lbs. $\$ 1.35,50$ lbs. $\$ 6.50,100$ lbs. $\$ 12.50$.

Violet or Green. One pound will cover about 1,300 square inches. Either color, lb. 30 cts., 10 lbs. $\$ 2.25$.

\section{Toothpicks}

Double-pointed. No. 1B. Box $10 \mathrm{cts}$., 3 boxes $25 \mathrm{cts}$, 17 boxes $\$ 1$; case (100 boxes) $\$ 5$.

Wired, White. Wired ready for use. $1,000,30$ cts.; box of 10,000 , $\$ 1.75$; case of $50,000, \$ 7.50$.

Wired, Green. Dyed with rustless dye. 1,000, 30 cts.; box of $10,000, \$ 2$; case of $50,000, \$ 8.75$.

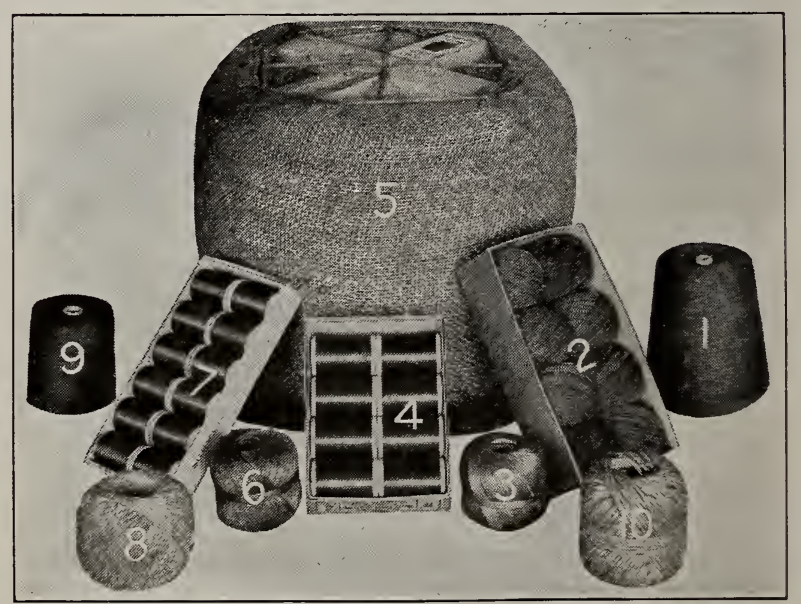

\section{Twines}

Silkaline. (4.) For stringing smilax. F, fine; FF, medium; FFF coarse. Specify which is desired. 2 -oz. spool 20 cts., $S$ spools 1 lb., $\$ 1.25$.

Florists' Thread, King Arthur. (7.) Similar to Silkaline Spool 20 cts.; box, 12 spools, $\$ 1.25$

Holly Green Cotton Twine. Lb. 30 cts., 5 lbs. $\$ 1.50$.

White Cotton. For store use and tying cut-flowers. Lb. $30 \mathrm{cts}$ 5-lb. pkg. $\$ 1.50$.

Green Cotton, on tubes. (9.) Lb. $40 \mathrm{cts}$. 


\section{TWINES, continued}

Sea Island Cotton. (1.) Serviceable Twine for ornamental purposes; strong. Cones containing about 2 lbs. Moss Green, cable-laid, lb. 60 cts.

Sea Island Cotton, in boxes. (2.) Soft finish and very neat for small packages. Eight balls to a box. Green or white. Box (1 lb.) $60 \mathrm{cts}$.

Hemp. A strong, dark twine for tying. No. 12. (6) Lb. 35 cts. No. 18. Lb. 30 cts. No. $4 \frac{1}{2}$. (10.) In balls, lb. 25 cts.

Nile Green, Linen Finish. (3.) For tying packages and stringing smilax. Lb. 60 cts.

Kraft Finish. (3.) Matches Kraft wrapping-paper. Lb. 60 cts. Tube Rope. (5.) 6-ply. Soft rope for heavy packing. Very serviceable. In $50-\mathrm{lb}$. reels. Lb. 18 cts.

Jute. In balls. (8.) 2- or 3-ply. For medium-weight packages. Lb. 19 cts., 10 lbs. $\$ 1.75$.

\section{Trowels}

Forged Steel, No. 211. Blade and shank in one piece. 6-inch, each 35 cts.; 7 -inch, 40 cts.; 8 -inch, 45 cts.

Forged Steel Berry Trowel, No. 211A. Each 35 cts.

Solid Steel, No. 212. Blade and shank in one piece. 6-inch, each 25 cts.

English Pattern, No. 215. Extra-heavy garden trowel. Blade and shank riveted by new method. 6-inch, each $12 \mathrm{cts}$.

Bulb Trowel. 6-inch blade, each 50 cts.

Transplanting, No. 217. Blade and shank made of one piece of heavy steel. 6-inch, each $12 \mathrm{cts}$; 7 -inch, each $18 \mathrm{cts}$.

Ladies' No. 216. Riveted. 6 -inch, each 5 cts., doz. 50 cts.

Imported English. Cast-steel and welded. A very fine Trowel. 6-inch, each \$1.10; 7-inch, each $\$ 1.25$.

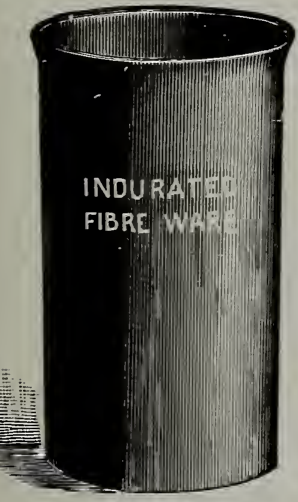

\section{Fibrotta Florists' Vases}

They are rich and handsome in appearance; easily cleaned; durable. Mahogany finish constantly in stock. Packed one-half dozen in crate. Always order by number.

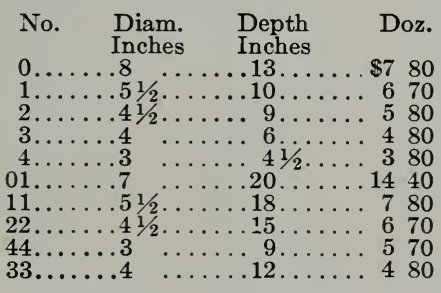

\section{Cemetery Vases, or Bouquet-Holders}

Tin, Annealed, white-enameled; durable. No. 2. Doz. $\$ 1.50$, $100, \$ 12$.

Glass, Clara. Bell-shaped. Holds sufficient water to keep flowers fresh for a long time. $91 / 2$-inch, each 15 cts., doz. $\$ 1.50 ; 12$-inch, each 25 cts., doz. $\$ 2.25$; 16 -inch, each 30 cts., doz. $\$ 3$.

Stoneware, Green. 10 inches high. Made of heavy, dark green, glazed stoneware. Each 20 cts., doz. \$2.

\section{Watering Pots}

Galvanized Iron. With round body, especially designed for greenhouse use; long spout; 2 copper-faced roses.

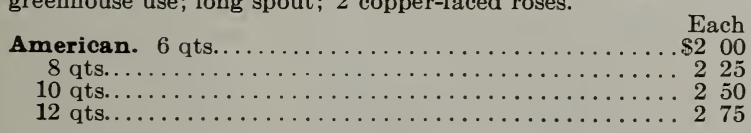

\section{Weeders}

Excelsior, each 10 cts., doz. 75 cts.

Hazeltine, each 20 cts., doz. \$2.

Lang's, each 20 cts., doz. $\$ 2$.

Norcross. 3-prong, Midget, each 35 cts. 3-prong, long-handled cultivator, each $50 \mathrm{cts}$. 5-prong, each 75 cts.

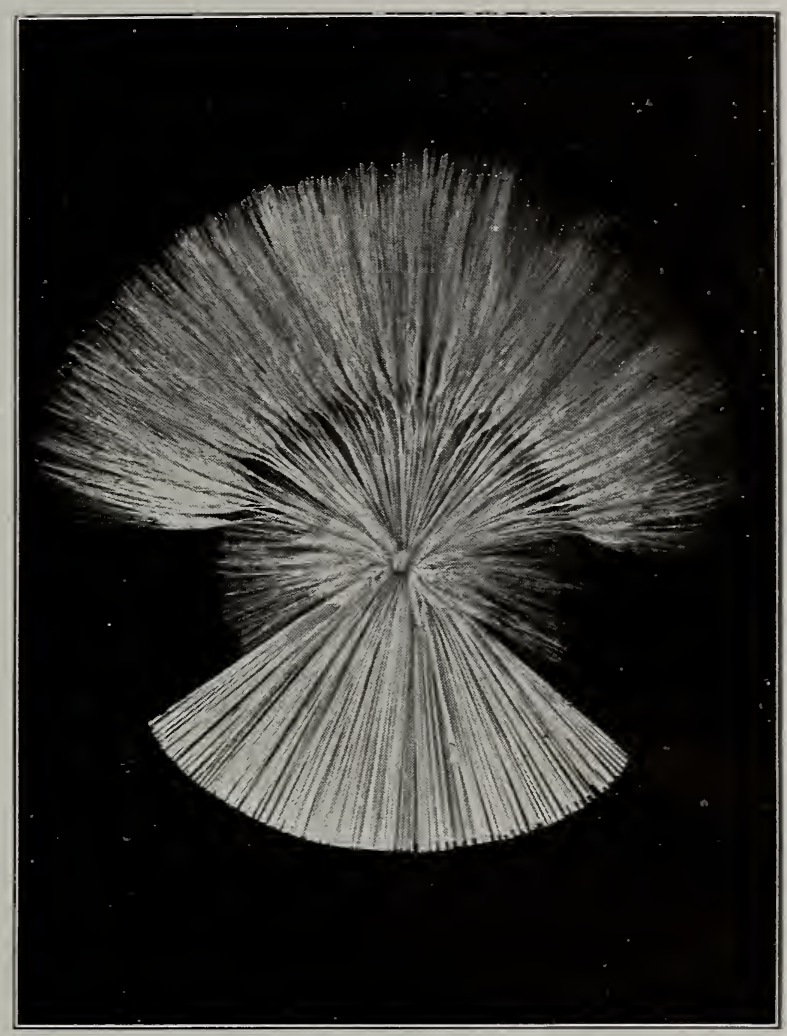

\section{Wheat Sheaves, Flat}

Our Sheaves are made of the best quality of selected Wheat. They are of full length and perfect in shape. Please order by number.

No. 4. 17-inch

No. 5. 18-inch.

No. 6. 19-inch.

No. 7. 22-inch. ...

No. 8. 25-inch. $261 / 2$-inch

No. 10. 28-inch.

Wheat, Loose. Bleached. Lb. 50 cts.

\section{Wheelbarrows}

Special Greenhouse. A well-made, steel-braced, narrow Wheelbarrow. Painted red. Inside dimensions of bed: width, handle end, 19 inches; length, 28 inches; depth, 12 inches. Shipping weight, 45 lbs. Each \$4.50.

Globe Garden. A medium-sized Barrow; box 30 inches long, 19 inches wide at wheel end, 24 inches at handle end; 12 inches deep, 20-inch steel wheel. Each \$4.

\section{Wire, Bright Annealed}

Prices subject to change without notice. Per lb. Perstone

No. 18 .

No. 20 .

No. 22

No. 24

No. 26 .

No. 28

No. 36 .

No. 18. Galvanized

so 12

$12 \quad 100$

$14 \quad 110$

$\begin{array}{lll}15 & 1 & 15 \\ 20 & 1 & 25\end{array}$

$20 \quad 135$

$30 \quad 210$

\section{Cut Wire in Wooden Boxes}

This is clean, extra-soft, white wire, straightened and cut in lengths of 12 and 18 inches, ready for immediate use. 12 lbs. to box.

No. 18
No. 20
No. 22
No. 24
No. 26
No. 28
No. 36 Per box so 95 110 130 145 175 225

See inside cover page for prices of Grass Seed for lawns and pleasure-grounds. Extra-choice seed. thoroughly cleaned, and packed in cartons for convenience in handling. 


\section{Florists' Wire Designs}

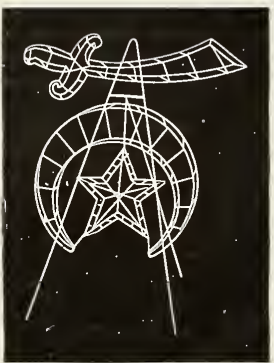

MYSTIC SHRINE
WE'ARE at all times able to wire designs. They are perfect in shape being made by accomplished workmen.

\section{FOR CASH IN 10 DAYS Special Cash Discounts on Wire Designs. \\ DISCOUNTS \\ On orders from $\$ 1.00$ to $\$ 10.0020$ per cent On orders from 10.00 to 20.0025 per cent On orders from 20.00 to 30.0030 per cent On orders over $30.0033 \quad 1-3$ per cent. \\ Write for Special Discount on Orders over $\$ 100$}

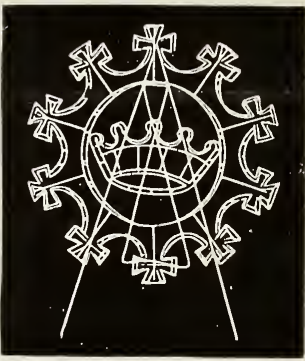

ROYAL ARCANUM

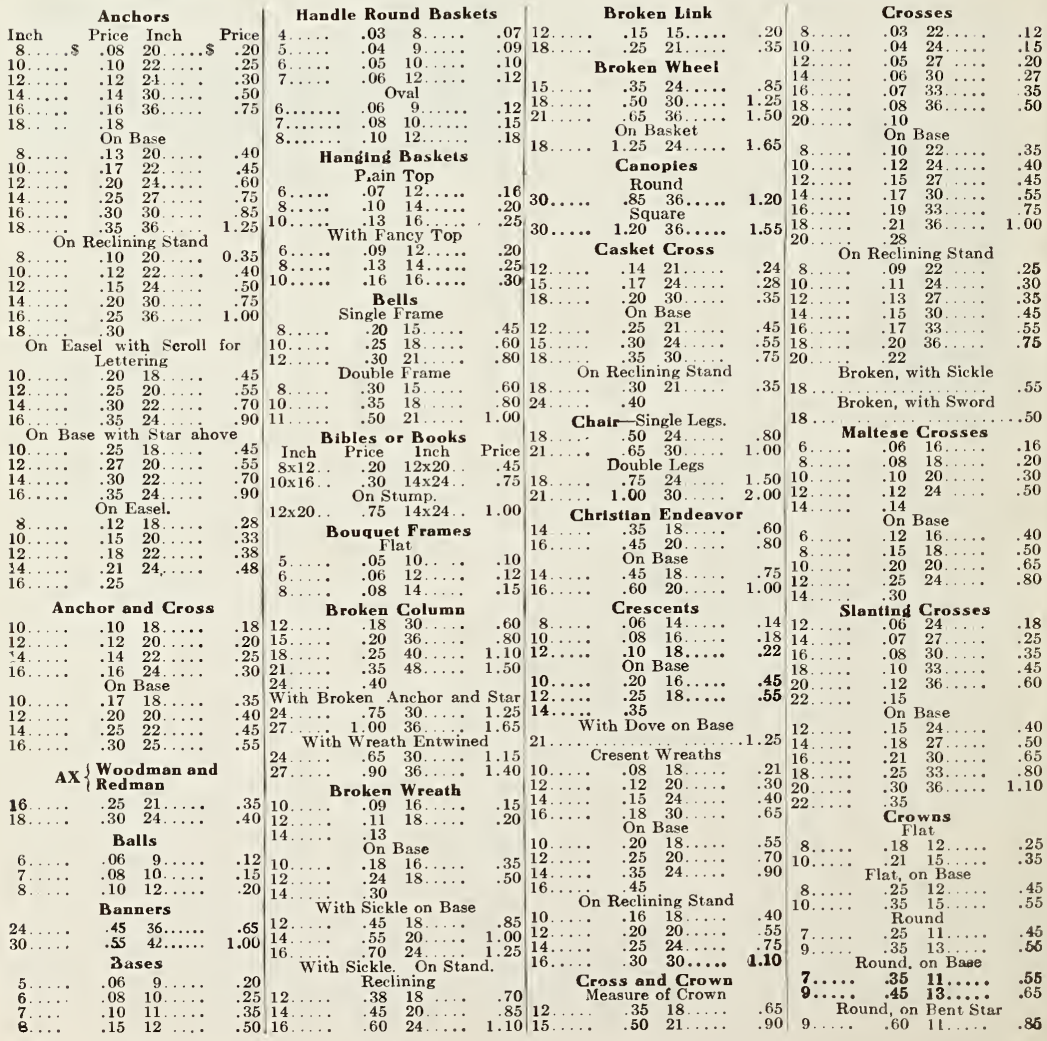




\section{FLORISTS' WIRE DESIGNS - Continued.}

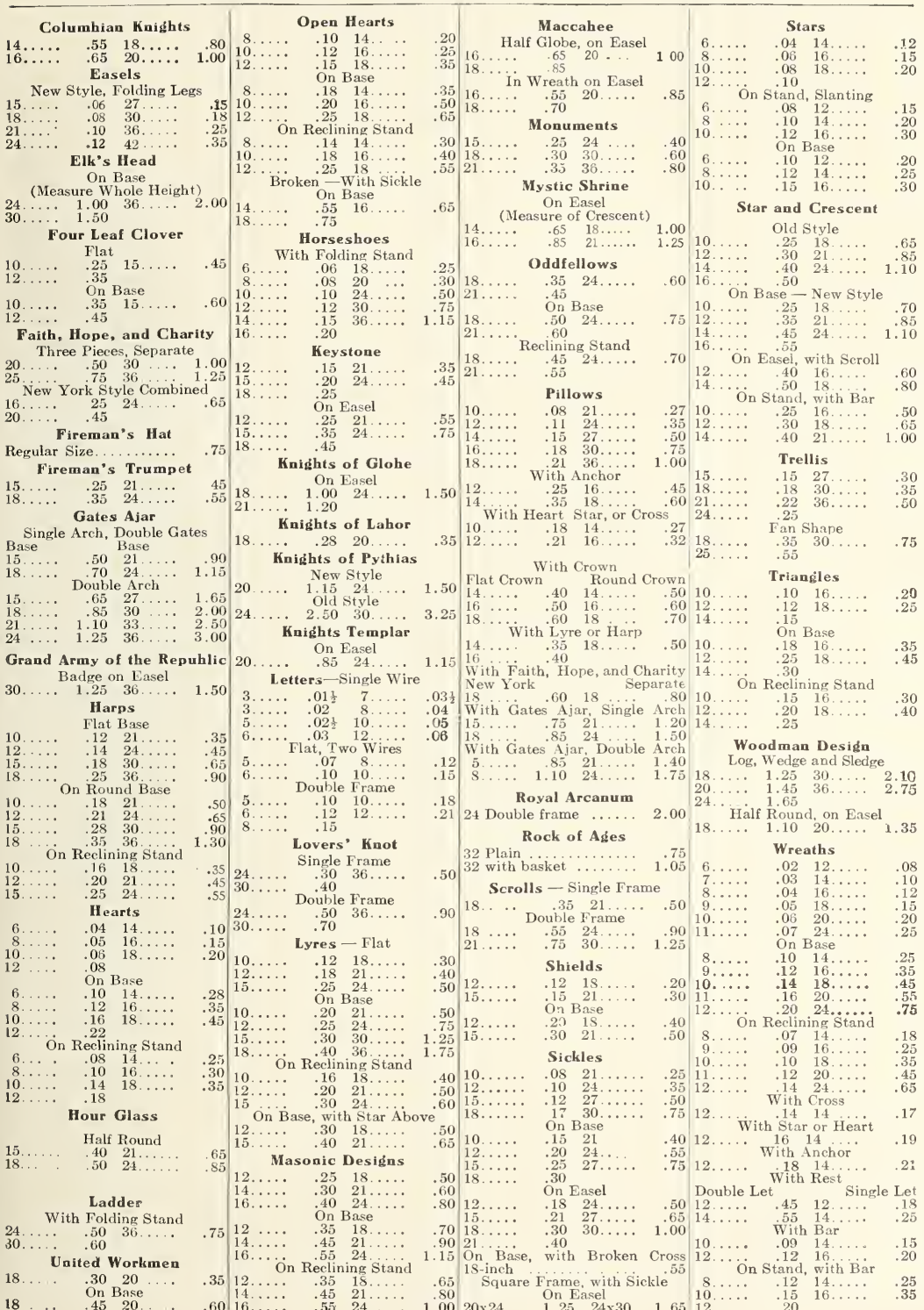




\section{BULBS}

\section{ROOTS}

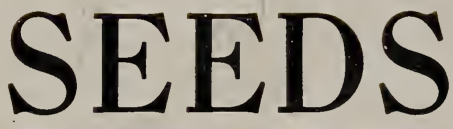

\section{For Fall Planting}

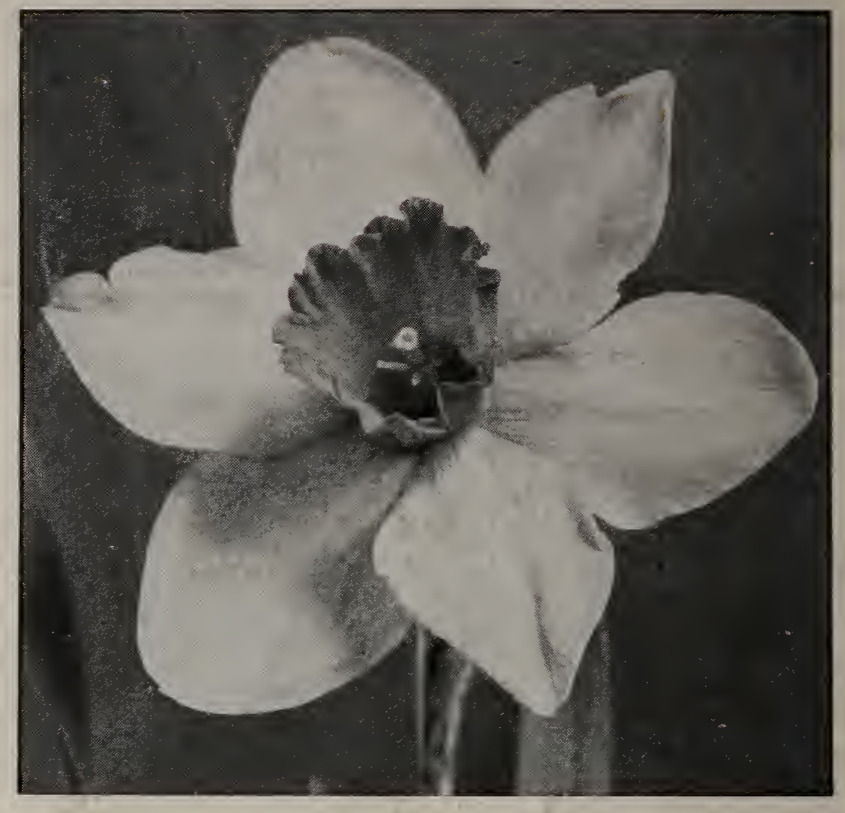

\section{(iv) \\ BULBS}

Each year we inport large quantities of Dutch Bulbs, Roman Hyacinths and French Narcissi from the famed growers in Europe; Lilium longiflorum and other varieties from Japan, and Lilium Harrisii from Bermuda. We secure our supply direct from the growers. Under present conditions we know it will be to your advantage if you place your orders early. Give us a list of your requirements and we shall be glad to quote prices.

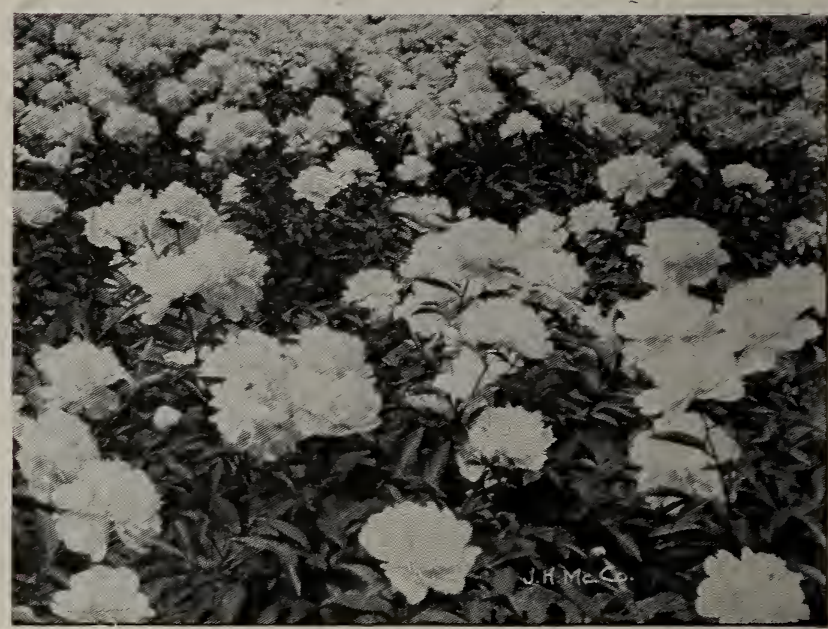

\section{ROOTS}

For several years we have made a specialty of Peonies, having a large tract of land devoted solely to growing the leading commercial sorts. Our Special Collection for Cutting is described in our Autumn Price-List, which will be ready about August I 5. Fall is the best time to replant Peonies.

\section{SEEDS}

We have an extra-good stock of Pansy seed for summer and fall sowing. Barnard's Florists' Mixture has become famous not only with the growers in Chicago and vicinity, but all over the country, proving to be the equal of any and superior to most Pansy seed mixtures.

Your attention is called to our line of seeds of Perennials. Every season shows an increasing demand for plants that live for several years, and the perennials are increasing in popularity every year. If you are prepared to furnish the plants, you can secure a nice trade and an increased profit.

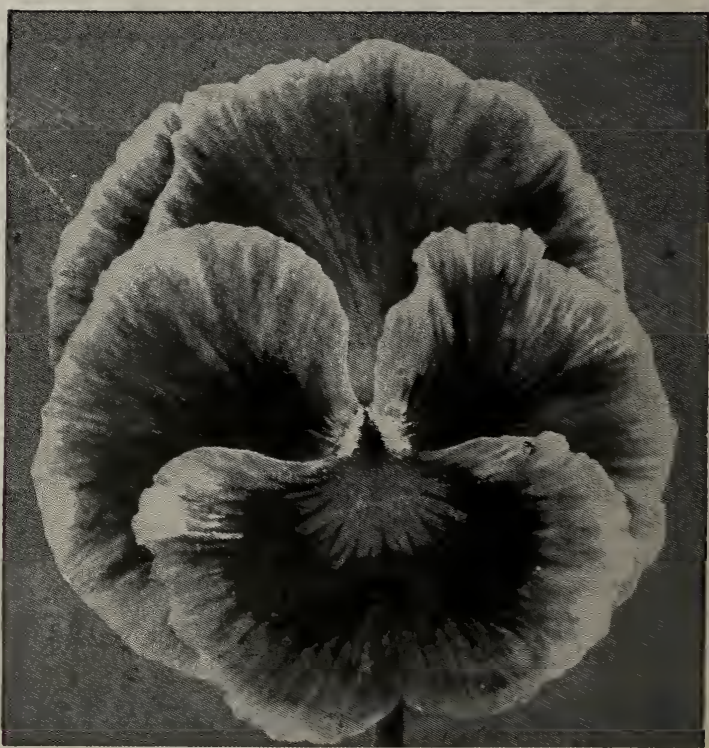

